\title{
ABSTRACT \\ STATE-OF-CHARGE ESTIMATION OF THE LITHIUM-ION BATTERY USING NEURAL NETWORK BASED ON AN IMPROVED THEVENIN CIRCUIT MODEL
}

This paper focuses on the real-time estimation of the State of Charge (SOC) in Lithium-Ion battery. When it comes to highly complex electrochemical reaction inside the battery, the conventional first order battery model is not accurate and cannot respond to the battery's conditions correctly because of the simplicity of the model, especially in the beginning and ending of charge/discharge stages. So, the neural network (NN) is selected to estimate the SOC dynamically due to its strong nonlinear fitting ability. The NN strategy also was used to implement the parameter identification for the battery model. In order to obtain accurate and robust SOC estimations, the correct order of the equivalent circuit model was chosen. SOC mean square error (MSE) method can determine the proper order of the battery model for the online SOC estimation. This paper compared the NN strategy with adaptive extended Kalman filter for the online SOC estimation. It shows that the proposed NN strategy can estimate the SOC of battery with high effectiveness and accuracy.

Haoliang Zhang

August 2018 



\title{
STATE-OF-CHARGE ESTIMATION OF THE LITHIUM-ION BATTERY USING NEURAL NETWORK BASED ON AN IMPROVED THEVENIN CIRCUIT MODEL
}

by

Haoliang Zhang

\author{
A thesis \\ submitted in partial \\ fulfillment of the requirements for the degree of \\ Master of Science in Electrical Engineering \\ in the Lyles College of Engineering \\ California State University, Fresno
}

August 2018 
(C) 2018 Haoliang Zhang 


\section{APPROVED}

For the Department of Electrical and Computer Engineering:

We, the undersigned, certify that the thesis of the following student meets the required standards of scholarship, format, and style of the university and the student's graduate degree program for the awarding of the master's degree.

Haoliang Zhang

Thesis Author

$\overline{\text { Woonki Na (Chair) } \quad \text { Electrical and Computer Engineering }}$

\begin{tabular}{ll}
\hline Peter Kinman & Electrical and Computer Engineering
\end{tabular}

\begin{tabular}{ll}
\hline Youngwook Kim & Electrical and Computer Engineering
\end{tabular}

For the University Graduate Committee:

Dean, Division of Graduate Studies 
AUTHORIZATION FOR REPRODUCTION

\section{OF MASTER'S THESIS}

I grant permission for the reproduction of this thesis in part or in its entirety without further authorization from me, on the condition that the person or agency requesting reproduction absorbs the cost and provides proper acknowledgment of authorship.

$\mathrm{X}$ Permission to reproduce this thesis in part or in its entirety must be obtained from me.

Signature of thesis author: 


\section{ACKNOWLEDGMENTS}

I wish to thank my major professor, Dr. Woonki Na most deeply for his support, guidance, and encouragement through my graduate study. I would like to thank to Dr. Jonghoon Kim who provided the experimental data and helped me improved the algorithm. I want to thank Dr. Nagy Bengiamin who helped me to enhance my ability of the system modelling. Also, I would like to thank Dr. Daniel Bukofzer who helped me to enhance my background of the mathematics and circuit analysis.

I would like to extend my heartfelt gratitude to my parents, Zhao Zhang and Chunxian Qu for their love, support, and encouragement while pursuing my course of study.

Last, but by no means least, I thank my fiancée, Jin Ren, for having allowed me the time and space, which I have needed over the last 2 years to complete my study without accompanied her. 


\section{TABLE OF CONTENTS}

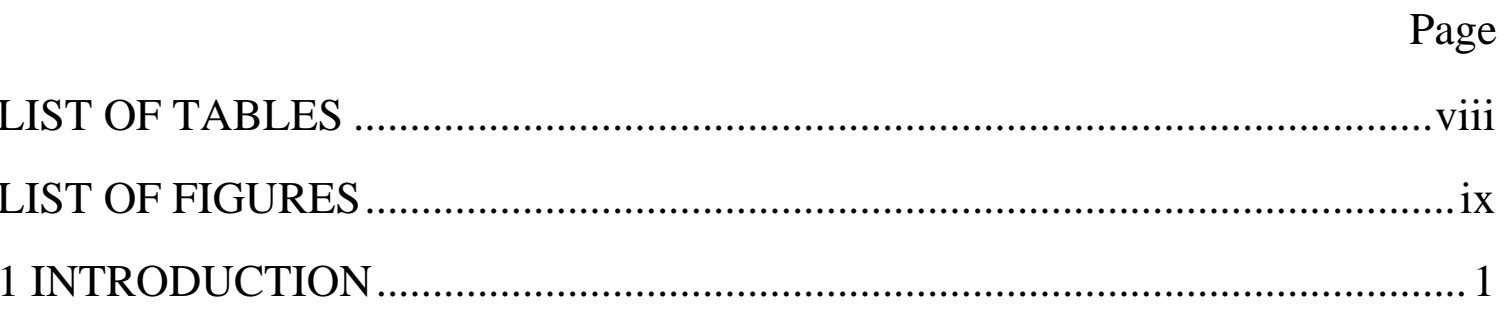

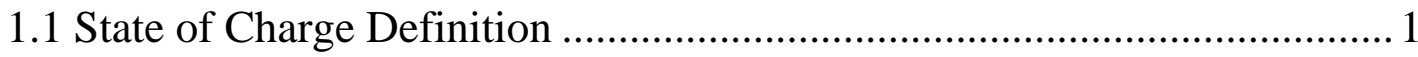

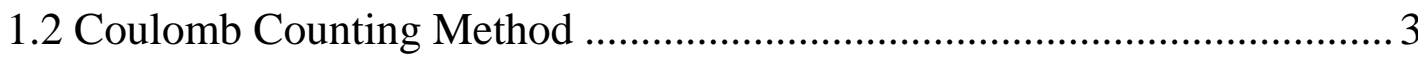

1.3 Open Circuit Voltage Measurements .......................................................... 4

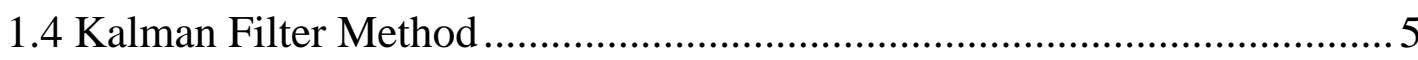

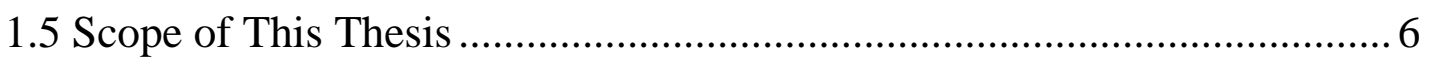

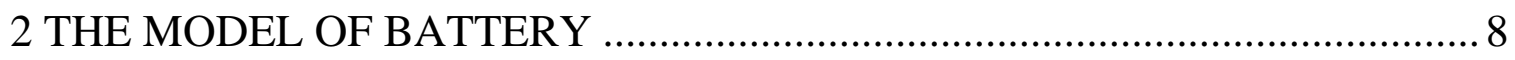

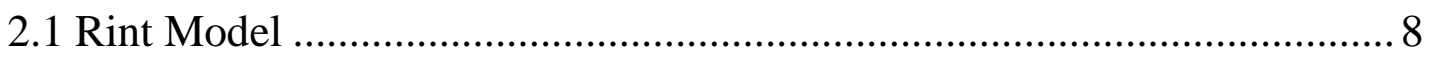

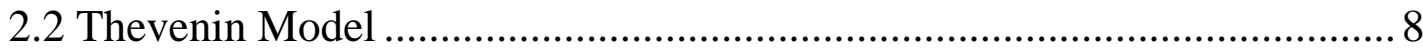

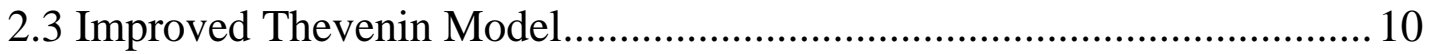

2.4 Variable-order RC Equivalent Circuit Model.......................................... 12

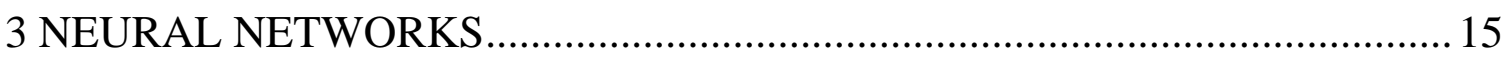

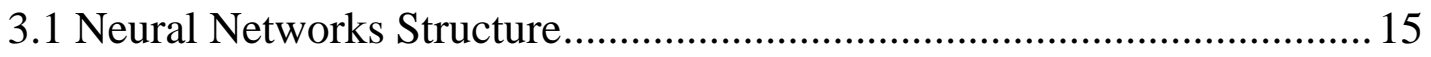

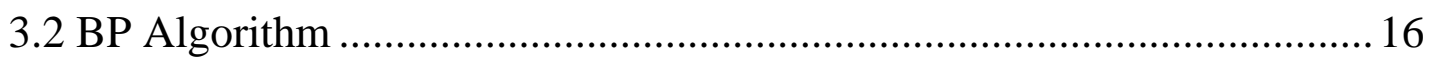

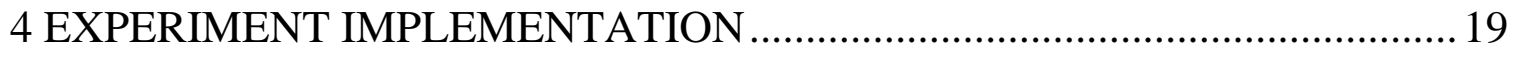

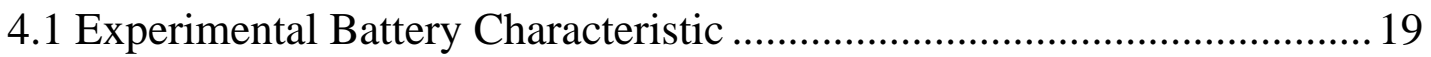

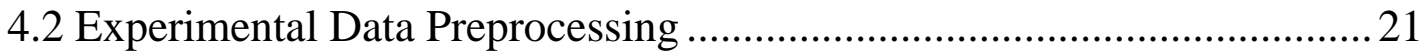

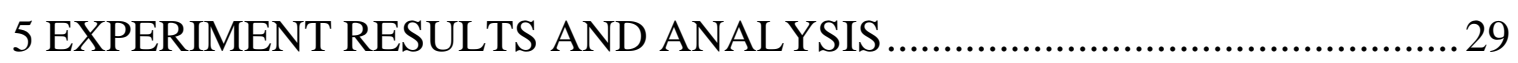

5.1 Battery Model Identification ...................................................................22

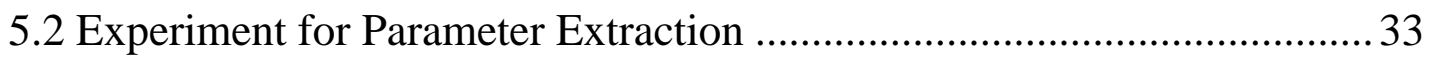

5.3 Thevenin Model Identification Based on Neural Network ....................... 37 
Page

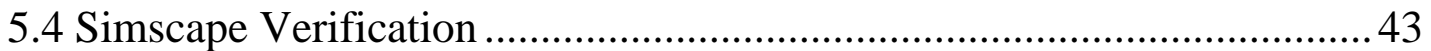

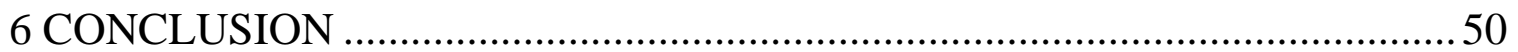

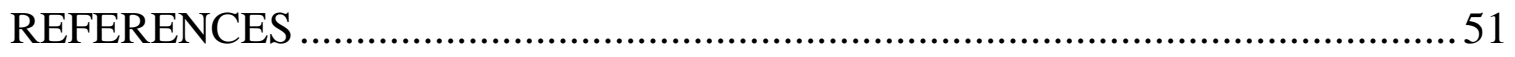

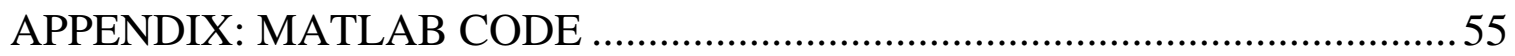




\section{LIST OF TABLES}

Page

Table 1-1 The number of cycles and relative capacity .........................................5

Table 4-1 The battery's voltage and K-means clustering voltage .........................22

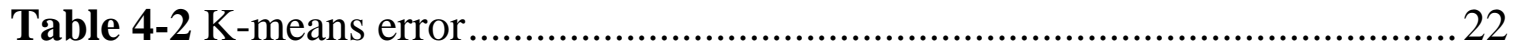

Table 4-3 OCV and SOC measurement value....................................................... 27 


\section{LIST OF FIGURES}

Page

Figure 1-1 Average Historical Annual Gasoline Pump Price ............................... 2

Figure 1-2 Voltage curves under different discharging current ........................... 4

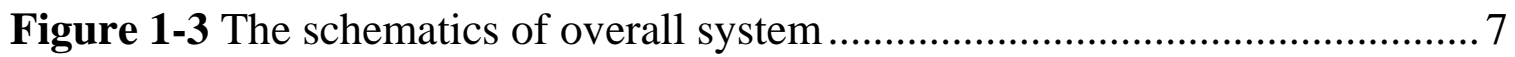

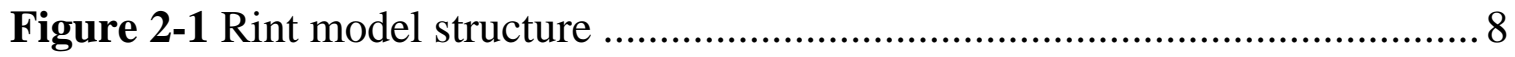

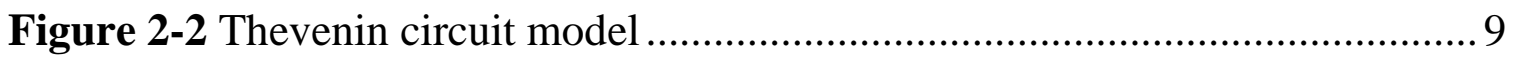

Figure 2-3 Improved Thevenin model structure.................................................. 11

Figure 2-4 Variable-order RC Equivalent Circuit Model ..................................... 13

Figure 3-1 Neural network structrue ……………......................................... 15

Figure 4-1 Thermal chamber and measurement equipment .................................. 19

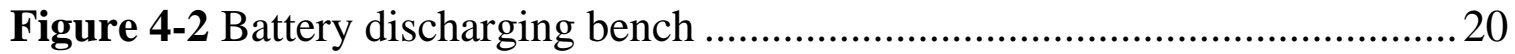

Figure 4-3 Experimental voltages of five batteries ............................................. 20

Figure 4-4 Experimental data of the battery's terminal voltage............................ 23

Figure 4-5 The SOC versus terminal voltage ……….......................................... 24

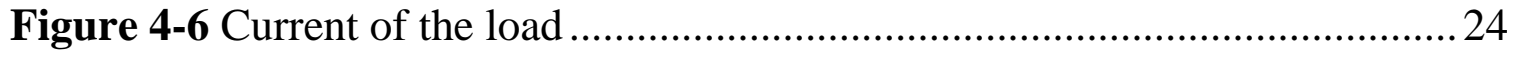

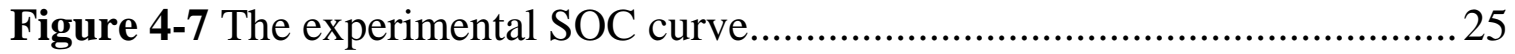

Figure 4-8 SOC versus OCV and terminal voltage …….....................................26

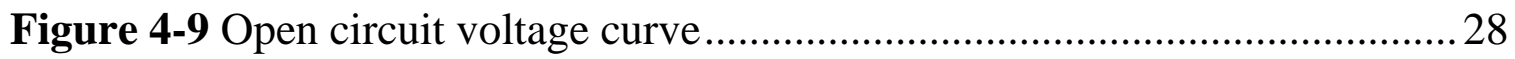

Figure 5-1 Battery's terminal voltage response …………………....................... 34

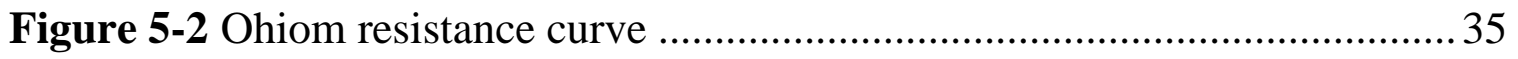

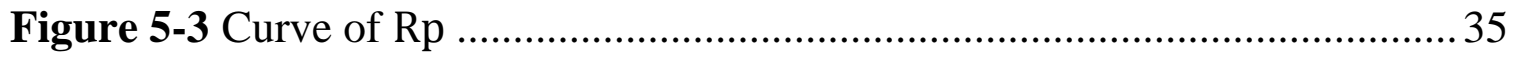

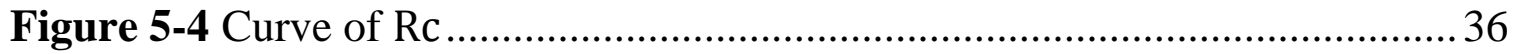

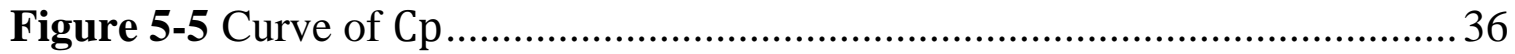

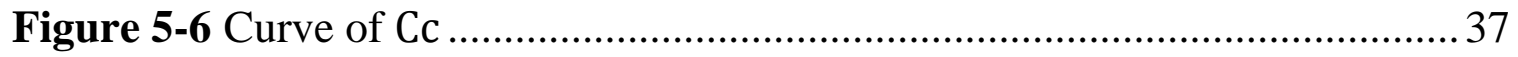


Figure 5-7 BP neural network for the Thevenin model........................................ 38

Figure 5-8 Flow chart of network algorithm ...................................................... 40

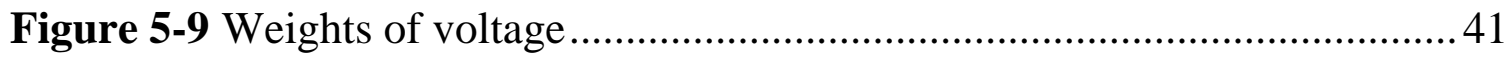

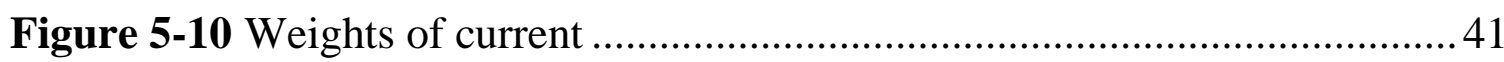

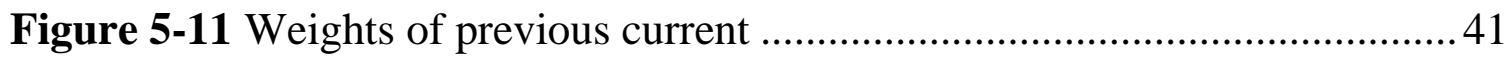

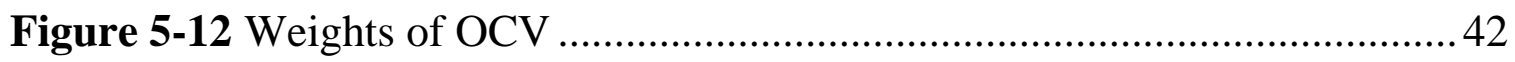

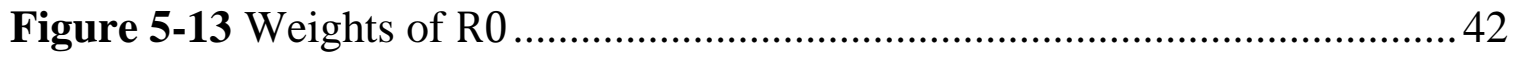

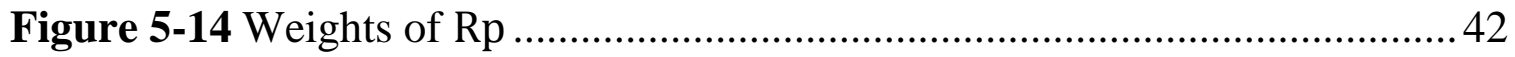

Figure 5-15 Fitted curve based on neural network............................................. 43

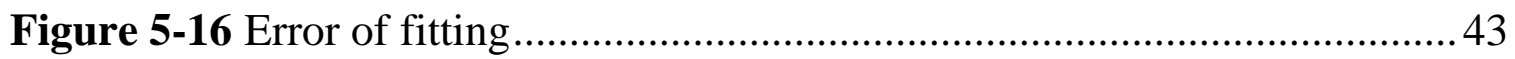

Figure 5-17 Simscape circuit of improved Thevenin model................................ 44

Figure 5-18 Simscape circuit of Thevenin model ............................................. 44

Figure 5-19 Created circuit components ............................................................. 44

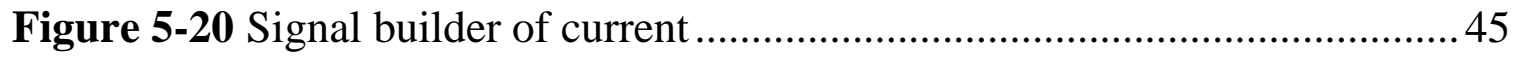

Figure 5-21 Simulation results of the Thevenin model and experiment data .......45

Figure 5-22 Estimation error of Thevenin model.............................................. 46

Figure 5-23 Simulation results of improved Thevenin model and

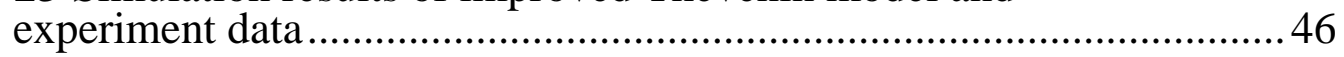

Figure 5-24 Estimation error of improved Thevenin model ............................... 47

Figure 5-25 Simulation results of variable Thevenin model and experiment data ................................................................................. 47

Figure 5-26 Estimation error of variable Thevenin model.................................... 48

Figure 5-27 Comparison of the actual SOC, the SOC estimation by EKF, and the SOC estimation by variable Thevenin model ....................................... 48

Figure 5-28 Comparison of SOC estimation error by proposed algorithm and $\mathrm{EKF}$ 


\section{INTRODUCTION}

Gasoline is the primary vehicle energy resource. Under the background of global energy shortage, the overall gasoline price has been kept rising since 1929. Figure 1-1 shows the average historical annual gasoline pump price. The gas price was almost two times than 20 years ago. In recent years, the pure electric vehicle becomes a popular study filed because of above reason and its high economic efficiency and lower environmental pollution [1]. Considering battery is the best power source for the next generation vehicle, and the accurate estimation of the battery performance is critical technic for the electronic vehicle operation [2]. Lithium-ion batteries have been widely used in many industrial fields. In order to use the Lithium-ion battery safely and efficiently, the internal states of the battery must be monitored appropriately in a timely manner [3]. For a Lithium-ion battery, migration of the electrolyte and the activity of electrode materials is affected by temperature, hysteresis, cells age and self-discharge rate [4]. Furthermore, the battery is not charge/discharge with the constant current. Usually, the battery used under complex conditions, temperatures, and even extreme environments.

\subsection{State of Charge Definition}

The safety of electric vehicle highly depends on Battery Management System (BMS). There are several factors can evaluate the batteries states. Especially, the accurate state-of-charge (SOC) is one of the most important factors of BMS [5]. Different from the common vehicle that can measure reaming gasoline by the float ball, the SOC of the electric vehicle cannot be measured directly but only be measured by external information of the battery [6]. The SOC is affected by the complicated internal nonlinear characteristics of the battery such as mobility of a charged particle, discharge current, battery age, etc. Furthermore, 


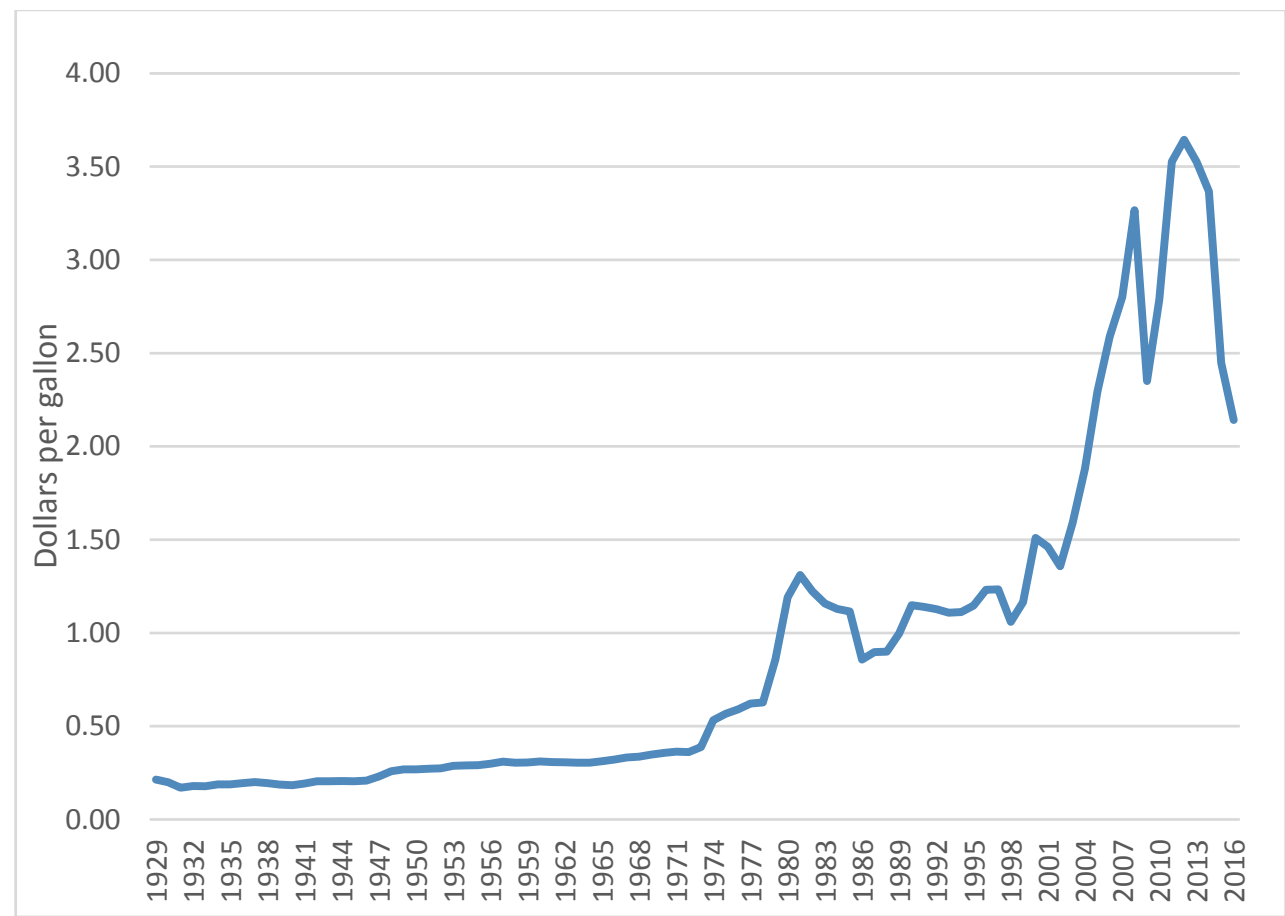

Figure 1-1 Average Historical Annual Gasoline Pump Price

the working temperature and external environment also make the real-time SOC measurement difficult.

SOC is the ratio of remaining capacity over the rated capacity. It can precisely represent the remaining time of the battery. Therefore, SOC measurement becomes the main method to analyze battery packs performance.

$$
\text { SOC }=\frac{Q_{\text {remain }}}{Q_{\text {rated }}} \times 100 \%
$$

Where,

$Q_{\text {remain }}$ is the remaining capacity of the battery.

$Q_{\text {rated }} \quad$ is rated capacity of the battery.

Separating the capacity into two parts, SOC can define as

$$
\mathrm{SOC}=\frac{Q_{\text {remain }}}{Q_{\text {discharged }}+Q_{\text {remain }}} \times 100 \%
$$

Where,

$Q_{\text {discharged }}$ is total charge release. 
Based on the equation (1.1) and (1.2), the conclusion can be drawn that SOC can represent the remaining time of the battery.

\section{$\underline{1.2 \text { Coulomb Counting Method }}$}

There are several SOC estimation methods was proposed. Coulomb counting method is a traditional SOC estimation approach, also known as current integration or ampere-hour method, it can estimate real-time SOC based on the last SOC status, involving in measuring the charge and discharging currents of a battery and integrating them over time to determine SOC. [7]

$$
\mathrm{SOC}=\operatorname{SOC}_{0}-\frac{1}{C_{N}} \int_{t_{0}}^{t} \eta I d t
$$

Where,

$S O C_{0}$ is the initial SOC

$C_{N} \quad$ is rated capacity

$\eta \quad$ is Coulomb efficiency during charging/discharging

I is real-time discharge current

Coulomb counting method is limited by specific factors such as temperature, battery history, charge, and discharge cycles. In addition, based on equation (1.3), accurate SOC highly depends on the accurate initial SOC and capacity. If the initial SOC and its capacity are not correlated, then it could lead to offsets in the estimated SOC. The inaccurate current value can add up over time, because of integration that causes the deviation between estimated SOC and the real SOC. Furthermore, the coulomb counting method not consider the selfdischarge or parasitic reactions in the battery. This shortcoming can be overcome by resetting the SOC [8]. Moreover, it is impractical to apply when the battery is operating in the changing environment. 


\subsection{Open Circuit Voltage Measurements}

There is a specific relationship between Open circuit voltage and battery SOC. The Open Circuit Voltage (OCV) is decreased when the battery is discharged, OCV is increased when the battery is charging. Therefore, the accurate SOC can be measured based on the OCV. But the accurate OCV only can be measured after the battery rest for a long time. If the rated voltage of the lithiumion battery is $3.7 \mathrm{~V}$, the OCV varies less than $1 \mathrm{~V}$ during operation. Especially, the OCV have only changed $0.3 \mathrm{~V}$ when SOC is between 0.4 0.7, this makes the SOC estimation difficult [9].

The main disadvantage of the battery is needs long period of rest to obtain accurate $\mathrm{OCV}$, so the open circuit voltage method cannot estimate real-time SOC accurately.

Figure 1-2 shows the example of equilibrium potential versus SOC curves. The lithium-ion battery is discharged at the constant current of 0.25 Coulomb Ampere (C A), 0.5C A and 1C A respectively in a real environment, and the test is conducted under the actual load, the battery is discharging from the full charge to $0 \%$ of SOC.

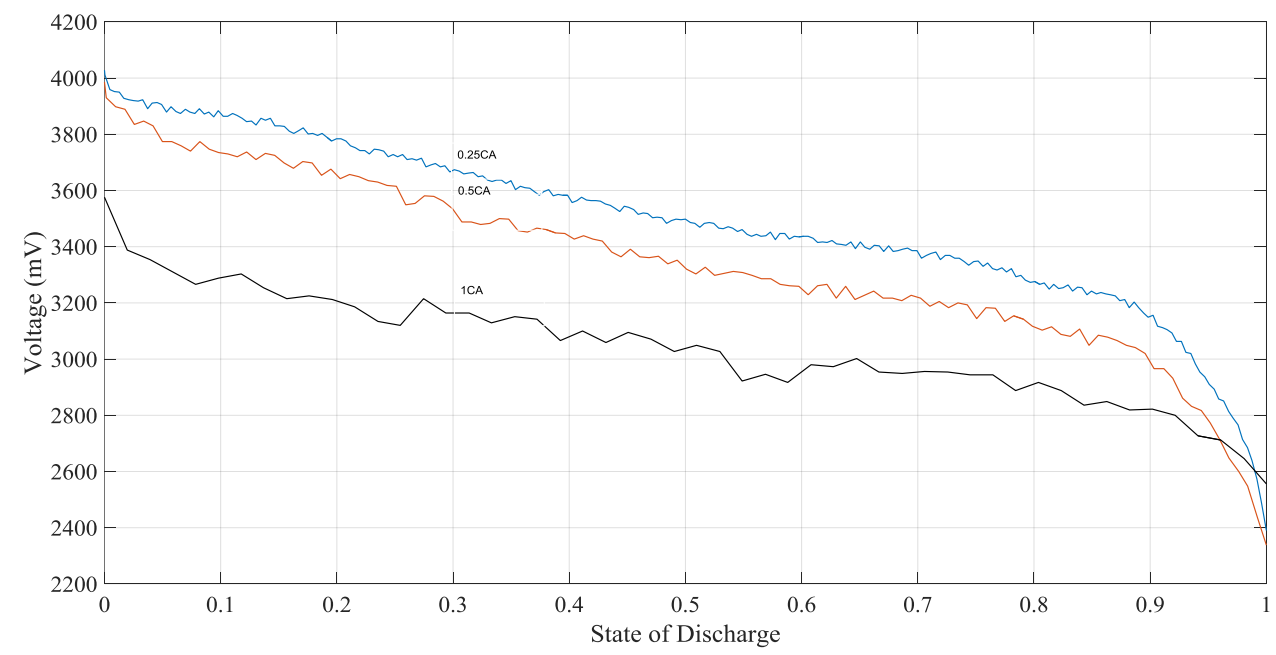

Figure 1-2 Voltage curves under different discharging current 
After numerous charge-discharge cycles, the internal material of the battery will be consumed and faded. The real capacity of the battery will decrease dramatically after hundreds of charge-discharge cycles.

Table 1-1 illustrates the relative capacity after the numbers of chargedischarge cycles. After 800 charge-discharge cycles, the real capacity of the battery reduced almost ten percent of the original capacity.

Table 1-1 The number of cycles and relative capacity

\begin{tabular}{c|c}
\hline Time & Relative capacity \\
\hline 100 & 0.995 \\
\hline 200 & 0.986 \\
\hline 400 & 0.962 \\
\hline 800 & 0.902 \\
\hline
\end{tabular}

\subsection{Kalman Filter Method}

The Mathematician R.E. Kalman proposed the Kalman filter in 1960. The Kalman filter is an algorithm that can be used in the linear system. Using minimum variance to estimate the observation state of the model, the desired signal and data can be retrieved based on the observation state. In the battery estimation process, the Kalman filter highly depends on the system model. The error of the system model leads to the SOC deviation. Lately, the extended Kalman filter was proposed to solve the above problem that Kalman filter is only suitable for the linear system [10]. In the extended Kalman filter, the first order Taylor series expansion is applied to the non-linear equation, and then use Kalman filter to estimate the observation state of the system. The main idea of the extended Kalman filter is to transfer the nonlinear system to linear system [11]. After linearizing the system, then process the data by minimum variance filter. In the study, measurement equation and observation equation are built, and then use 
the extended Kalman filter to estimate the SOC. Similar to Kalman filter, the extended Kalman filter also highly depends on the accurate model.

A Kalman filter model is

$$
\begin{gathered}
X_{t}=A_{t, t-1} X_{t-1}+W_{t} \\
Z_{t}=C_{t} X_{t}+V_{t}
\end{gathered}
$$

Where,

$X_{t} \quad$ is the states of system at time $t$

$A_{t, t-1}$ is the transformational matrix

$Z_{t} \quad$ is the observation matrix

$W_{t}, V_{t}$ are white gaussian noise with covariance $N(0, R)$ and $N(0, Q)$

The process of a Kalman filter can be described in equation (1.6) to (1.7)

$$
\begin{aligned}
& \hat{X}_{t, t-1}=A_{t, t-1} \hat{X}_{t-1} \\
& P_{t, t-1}=A_{t, t-1} P_{t-1} A_{t, t-1}^{T}+Q \\
& H_{t}=P_{t, t-1} C_{t}^{T}\left[C_{t} P_{t} C_{t}^{T}+R\right]^{-1} \\
& \hat{X}_{t}=\hat{X}_{t, t-1}+H_{t}\left[Z_{k}-C_{k} \hat{X}_{t, t-1}\right] \\
& P_{t}=\left[I-H_{t} C_{t}\right] P_{t, t-1}
\end{aligned}
$$

Where

$P_{t, t-1}$ is the covariance

$H_{t}$ is the filter gain

\subsection{Scope of This Thesis}

The overall system diagram is shown in figure 1-3. There are four main steps in this paper. (1) battery parameters identification, (2) select the suitable 
battery model based on MSE, (3) calculating the OCV by the decided battery model, (4) using the OCV-SOC function to estimate the SOC.

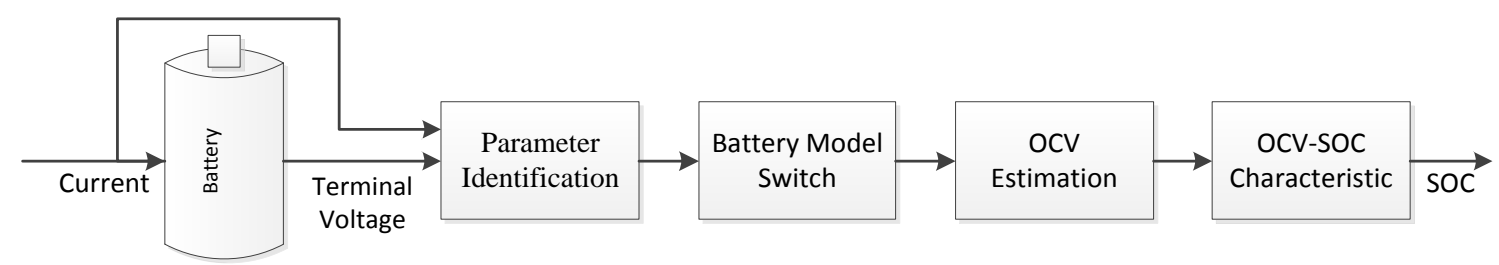

Figure 1-3 The schematics of overall system

In this paper, OCV, terminal cell voltage, and impedance at specific frequencies are all used to estimate the battery's SOC. This study used the linear NN to identify the model parameters. Once the parameters of battery model are identified, the correct battery model order can be chosen to estimate the SOC. The proposed method can reduce the amount of calculation and give the best SOC estimation for the Lithium-Ion battery during the charge/discharge operations. 


\section{THE MODEL OF BATTERY}

The charge/discharge process of the battery is a complicated electrochemical process [12]. This makes the real-time SOC estimation very difficult. For a Lithium-ion battery, there is a variety of battery models available. The accurate battery model is key to estimating SOC.

\subsection{Rint Model}

Figure 2-1 shows Rint model structure [13]. The Rint model has a simple structure, $U_{o c}$ is OCV of the battery, $R_{0}$ is the internal resistance of the battery, $\mathrm{U}$ is the terminal voltage. A Rint model cannot represent the polarization reaction of the battery during the charge/discharge operation. Battery consists of an ideal voltage source and a resistance in the Rint model.

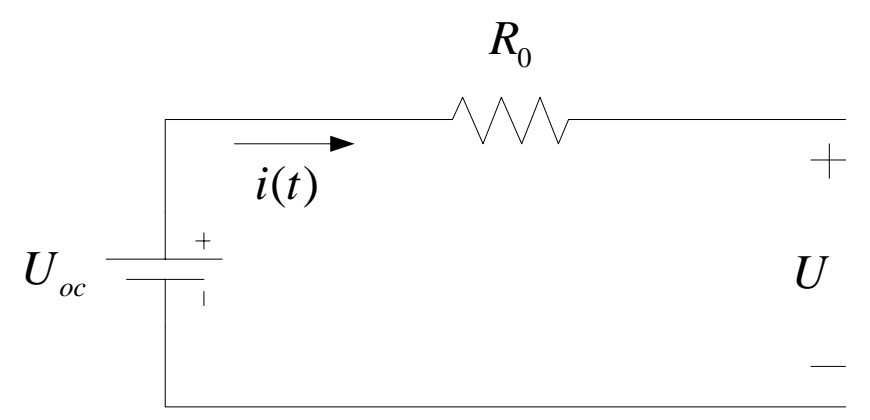

Figure 2-1 Rint model structure

\subsection{Thevenin Model}

In order to solve the problem that Rint model cannot reflect the polarization reaction, a Thevenin model was proposed. Figure 2-2 shows the Thevenin model structure [14]. Compared with the Rint model, the Thevenin model considers polarization resistance $R_{p}$ and polarization capacitor $C_{p} . U_{p}$ is the voltage across the capacitance $C_{p}, i(t)$ is charge/discharge current. The added resistance and capacitor pair make the battery model more accurate. The Thevenin can accurately 
estimate SOC of battery when battery charge/discharge in the stable state, which usually happened during $0.2 \mathrm{SOC}$ to $0.8 \mathrm{SOC}$.

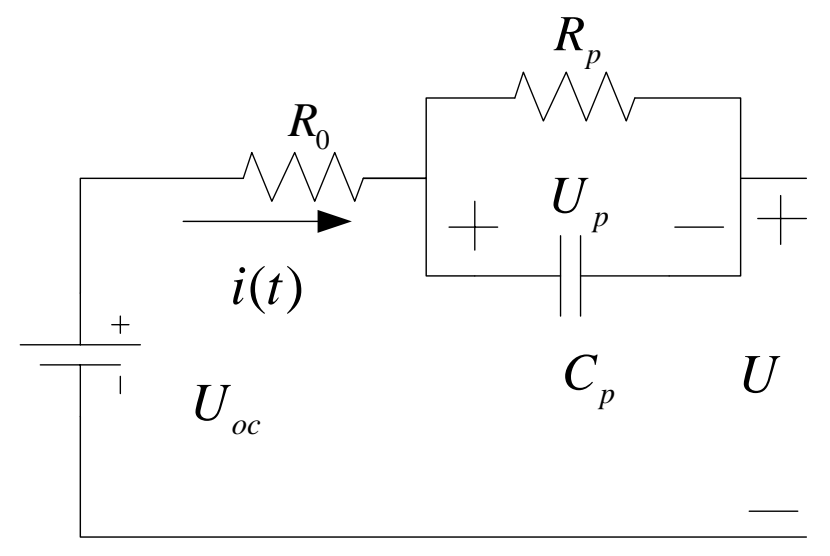

Figure 2-2 Thevenin circuit model

The electrical behavior of Thevenin model can be expressed as follows,

$$
\begin{gathered}
\frac{d U_{p}(t)}{d t}=-\frac{U_{p}(t)}{R_{p} C_{p}}+\frac{i(t)}{C_{p}} \\
U(t)=U_{o c}(t)-R_{o} i(t)-U_{p}(t)
\end{gathered}
$$

There are many discrete methods for the Thevenin model, such as backward difference method [15], forward difference method and bilinear transform method [16]. On the assumption that

$$
\frac{\mathrm{d} U_{o c}(t)}{\mathrm{dt}}=\left(U_{o c}(t)-U_{o c}(t-\Delta t)\right) / \Delta t \approx 0
$$

When the battery undergoes slow charge/discharge process, the open circuit voltage $U_{o c}(t)$ only has a slight change at a short sample period, then taking derivative of $U(t)$ 


$$
\begin{aligned}
U(k)= & \frac{R_{p} C_{p}}{T+R_{p} C_{p}} U_{L}(k-1)-\frac{R_{o} R_{p} C_{p}+R_{o} \mathrm{~T}+\mathrm{T} R_{p}}{R_{p} C_{p}+T} i_{L}(k) \\
& +\frac{R_{o} R_{p} C_{p}}{T+R_{p} C_{p}} i_{L}(k-1)+\frac{T}{T+R_{p} C_{p}} U_{o c}(k)
\end{aligned}
$$

Let,

$$
\begin{gathered}
a_{1}=\frac{R_{p} C_{p}}{T+R_{p} C_{p}} \\
b_{1}=-\frac{R_{o} R_{p} C_{p}+R_{o} \mathrm{~T}+\mathrm{T} R_{p}}{R_{p} C_{p}+T} \\
c_{1}=\frac{R_{o} R_{p} C_{p}}{T+R_{p} C_{p}} \\
d_{1}=\frac{T}{T+R_{p} C_{p}}
\end{gathered}
$$

The parameters of Thevenin model can be given by equation (2.9) to (2.11)

$$
\begin{gathered}
R_{o}=\frac{c}{a} \\
R_{p}=\frac{-a b-c}{a(1-a)} \\
C_{p}=\frac{T a^{2}}{-a b-c}
\end{gathered}
$$

Where,

$\mathrm{T} \quad$ is the sampling interval.

\subsection{Improved Thevenin Model}

However, the internal battery is a complicated process involving battery temperature, current intensity, battery health and more unpredictable factors. SOC 
of the battery shows strong nonlinear characteristics at the beginning and end of charge/discharge operation [17].

In order to estimate the SOC more accurate, the Improved Thevenin model is considered. Figure 2-3 shows the second order Thevenin model that adds another RC branch to the Thevenin model. In figure 2-3, $C_{c}$ and $R_{c}$ are concentration polarization capacitance and concentration polarization resistance respectively. $U_{c}$ is the voltage across the concentration polarization capacitance [18].

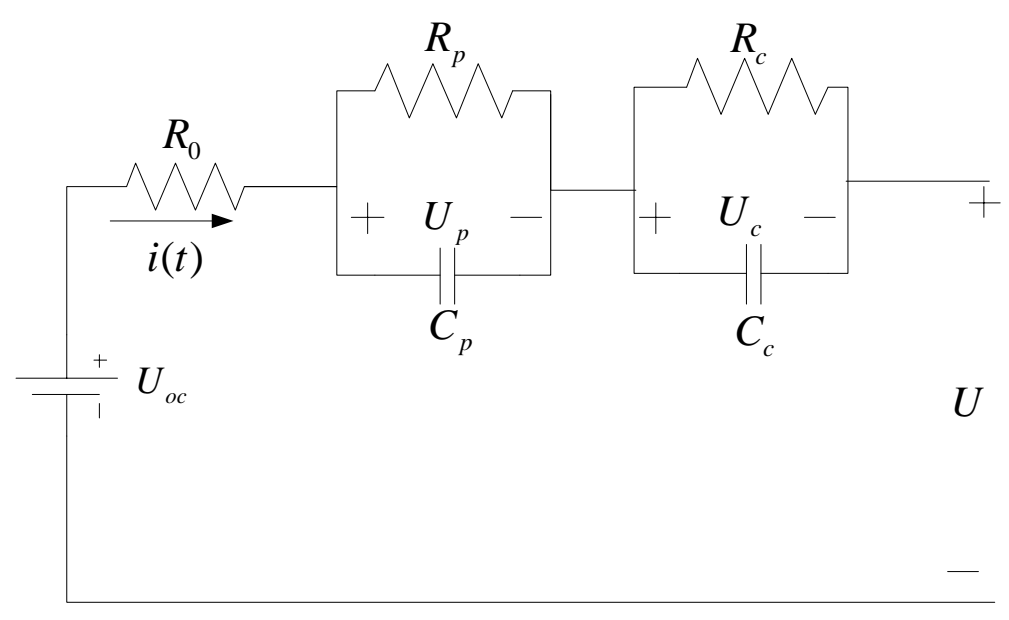

Figure 2-3 Improved Thevenin model structure

Similar to the improved Thevenin model, the mathematic expressions of the second order model are represented as follows.

$$
\begin{gathered}
\frac{\mathrm{d} U_{p}(t)}{\mathrm{dt}}=-\frac{U_{p}(t)}{R_{p} C_{p}}+i_{L}(t) / C_{p} \\
\frac{\mathrm{d} U_{c}(t)}{\mathrm{dt}}=-\frac{U_{c}(t)}{R_{c} C_{c}}+i_{L}(t) / C_{c} \\
\mathrm{U}(t)=U_{o c}(t)-R_{o} i_{L}(t)-U_{p}(t)-U_{c}(t)
\end{gathered}
$$


Assuming $\frac{\mathrm{d} U_{o c}(t)}{\mathrm{dt}} \approx 0$, taking the time the derivative of $U_{L}(t)$ gives

$$
\begin{aligned}
\frac{\mathrm{d} U_{L}(t)}{\mathrm{dt}}= & -\frac{1}{R_{c} C_{c}} U_{L}(t)+\left[\frac{1}{R_{p} C_{p}}-\frac{1}{R_{c} C_{c}}\right] U_{p}(t) \\
& -\left[\frac{R_{o}}{R_{c} C_{c}}+\frac{1}{C_{c}}+\frac{1}{C_{p}}\right] i_{L}(t)+\frac{1}{R_{c} C_{c}} U_{o c}-R_{o} \frac{\mathrm{d} i_{L}(t)}{\mathrm{dt}}
\end{aligned}
$$

Based on the equation (2.15) using backward difference equation, then.

$$
\begin{gathered}
U_{L}(k)=a_{1} U_{L}(k-1)+a_{2} U_{L}(k-2)+a_{3} I(k)+a_{4} I(k-1) \\
+a_{5} I(k-2)+a_{6}
\end{gathered}
$$

Among all estimation methods, equivalent circuit models are widely selected, which includes the Rint model [19], the Thevenin model [20], the RC model, and the partnership for a new generation of vehicle (PNGV) model [21]. The first order Thevenin model was widely used in the past because of its reliability when the battery is discharged with stable current and voltage. Moreover, by considering highly complex electrochemical reactions inside of the cell, the first order battery model cannot reflect the battery's condition accurately during the beginning and end of the operation process. Because of this reason, the improved Thevenin model was proposed which is the second order of Thevenin model. Comparing with the first order, the second order Thevenin model adds the other RC pair to the first order model due to the polarization characteristics of the battery [22].

\subsection{Variable-order RC Equivalent Circuit Model}

The accurate SOC estimation depends on reliable and practicable battery model. Generally, a good battery model has two main characteristics: (1) the model can represent the dynamic and static states accurately (2) the model has a low complexity [23]. The real-time order of the battery model is unknown, so it is 
hard to determine the real-time battery's SOC. In this paper, the SOC estimation is based on either first or second order battery equivalent model, use MSE to determines the battery model in real-time.

In figure 2-4, $R_{0}, R_{p a}, C_{p a}$ are the Ohmic resistance, polarization resistance, and the capacitance. $C_{p c}$ and $R_{p c}$ are concentration polarization capacitance and concentration polarization resistance, $Q_{1}$ is a switch that determines the model could be first order or second order.

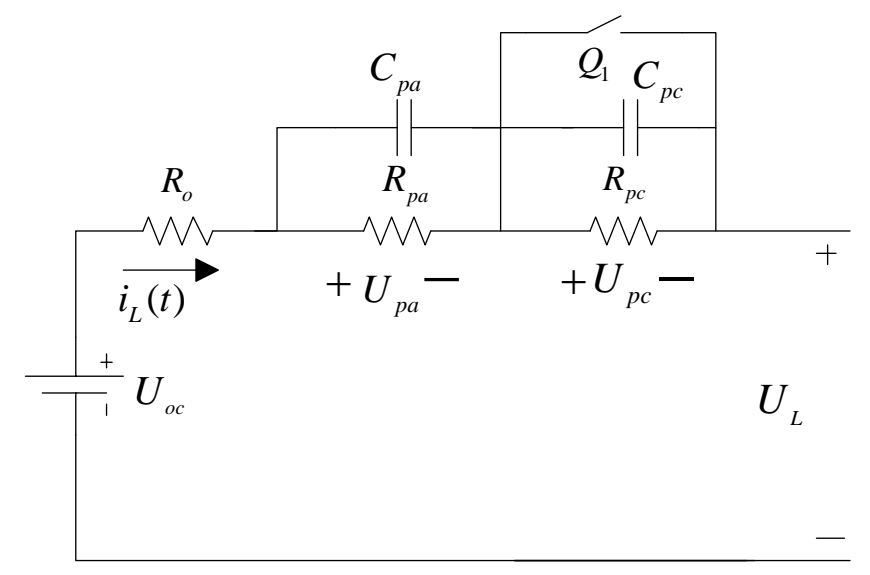

Figure 2-4 Variable-order RC Equivalent Circuit Model

The other variable-order battery model requires the tremendous computing workload such as AIC strategy. Based on the reference [24] The AIC can represent as

$$
\mathrm{AIC}=\log \left(\sigma^{2}\right)+\frac{2 \mathrm{M}}{\mathrm{T}}
$$

Where $\sigma^{2}$ is maximum estimation error, $\mathrm{M}$ is the number of parameters in the model, and $\mathrm{T}$ is the number of observations [25]. The first part is the parameter of goodness-of-fit of the model; the second part is called penalty function of overfitting. 


$$
\sigma^{2}=\frac{\mathrm{SSE}}{\mathrm{T}}=\frac{\sum\left(U_{L}-\widehat{U}_{L}\right)}{T}
$$

As a discharge period, for example, the RC loop of first order Thevenin model,

$$
\begin{gathered}
R_{p a} C_{p a} \frac{d U_{p a}(t)}{d t}+U_{p a}(t)=0 \\
U_{p a}(\mathrm{t})=U_{p a}\left(t_{0}\right) \mathrm{e}^{-\frac{\mathrm{t}}{R_{p a} C_{p a}}}
\end{gathered}
$$

The $U_{p a}\left(t_{0}\right)$ is the voltage. When the system stable, $U_{p a}\left(t_{0}\right)=R_{p a} i_{L}$

$$
U_{L}(t)=U_{o c}(t)-R_{p a} i_{L} \mathrm{e}^{-\frac{\mathrm{t}}{R_{p a} C_{p a}}}
$$

For figure 2- 4, if the model is the second order, similarly,

$$
U_{L}(t)=U_{o c}(t)-R_{p a} i_{L} \mathrm{e}^{-\frac{\mathrm{t}}{R_{p a} C_{p a}}}-U_{L}(t)=U_{o c}(t)-R_{p c} i_{L} \mathrm{e}^{-\frac{\mathrm{t}}{R_{p c} C_{p c}}}
$$

When AIC is minimized. The best performance of the model is achieved. And if the second part of AIC get few effects by M, in order to avoid this situation, use

$$
\mathrm{BIC}=\log \left(\sigma^{2}\right)+\frac{\operatorname{Mlog}(\mathrm{T})}{\mathrm{T}}
$$

Equation (2.23) is similar to AIC equation, the different is penalty item, which is clear that if $\mathrm{T}>7$, the penalty of BIC is greater than AIC.

Finally,

$$
\mathrm{BIC}=\log \left(\frac{\sum\left(U_{L}-\widehat{U}_{L}\right)}{T}\right)+\frac{\operatorname{Mlog}(\mathrm{T})}{\mathrm{T}}
$$




\section{NEURAL NETWORKS}

\subsection{Neural Networks Structure}

In the middle of the 1980s, Rumelhart and McClelland [26] proposed Multilayer Feedforward Neural Networks. Then the neural networks were used in many important areas and demonstrate great success.

The structure of a neural network is shown in figure 3-1. $U$ is $n$ dimensions input vector of neural networks, $Y$ is $m$ dimensions output vector of neural networks. Each neural node is represented by the circle. The general networks were constituted by three parts: input layer, hidden layer and output layer. Hidden layer can be one layer or several layers. Figure 3-1 only shows the neural networks that have one input layer, hidden layer, and one output layer, each layer is connected by different weight. The data used to train neural networks are $n$ dimensions input and $m$ dimensions output pairs that have length $L$.

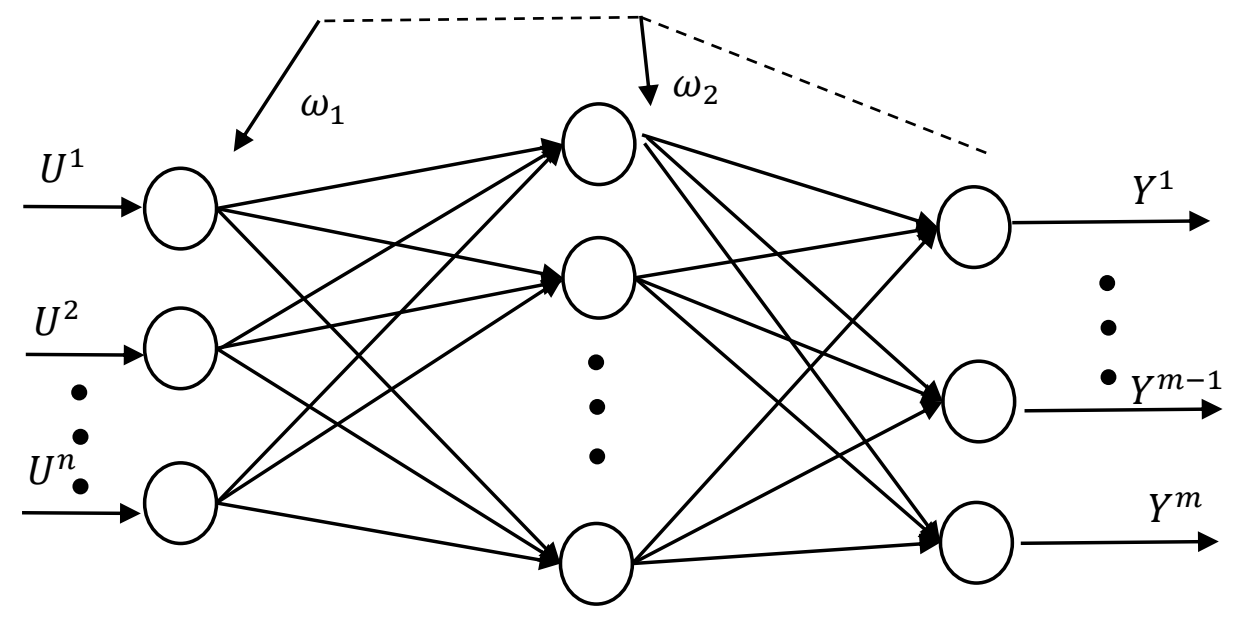

Input layer node Hide layer node Output laver node

Figure 3-1 Neural network structrue 


\subsection{BP Algorithm}

The BP learning algorithm has two main parts, forward propagation and reverse propagation [27]. In forward propagation, the input signal is transmitted to the output layer through hidden layer, the program will stop if the output layer has desired value. Otherwise, the algorithm will go to backpropagate. Backpropagate is the transmitted error signal through the same path that the signal is from, adjusting the weight and threshold value of each layer for decreasing data error.

The steps are as follows,

(1) Set the initial weight $w_{0}$ to a small random value between 0 and 1.

(2) Giving the input/output pairs, assuming the Pth set of samples input,

$$
\boldsymbol{u}_{p}=\left[\mathrm{u}_{1 p}, \mathrm{u}_{2 p}, \mathrm{u}_{3 p}, \cdots \mathrm{u}_{n p}\right]^{T}
$$

Similar Pth desired output,

$$
\boldsymbol{d}_{p}=\left[d_{1 p}, d_{2 p}, d_{3 p}, \cdots d_{m p}\right]^{T}, \mathrm{p}=1,2, \cdots, \mathrm{L}
$$

The output of node $i$ is $y_{i p}$ when Pth set of samples given, mean

$$
y_{i p}(t)=f\left[x_{i p}(t)\right]=f\left[\sum_{j} \omega_{i j}(t) I_{i p}\right]
$$

In equation (3.3) $I_{i j}$ is the $j t h$ output of node $i$, when the Pth signal is given.

$f(\cdot)$ is differentiable action function,

$$
f(x)=\frac{e^{x}-e^{-x}}{e^{x}+e^{-x}}
$$

Calculate objective function $J$

Assuming,

$$
E_{p}(t)=\left\|\boldsymbol{d}_{p}-\boldsymbol{y}_{i p}(t)\right\|_{2}=\frac{1}{2} \sum_{k}\left[d_{k p}-y_{k p}(t)\right]^{2}=\frac{1}{2} \sum_{k} e_{k p}^{2}(t)
$$


In equation (3.5), $y_{k p}$ is the output of $k$ th neural node after $t$ times weight adjusting when Pth input signal is given.

The final objective function of network $J$ is decision function.

$$
J(t)=\sum_{p} E_{p}(t) \leq \varepsilon
$$

If the equation (3.6) was satisfied, the program finished. Otherwise, the reverse propagation uses.

In Reverse propagate, the weight can be adjusted by the gradient descent, the $(t+1) t h$ weight between neural node $j$ and neural node $i$ can express as

$$
\omega_{i j}(t+1)=\omega_{i j}(t)-\eta \frac{\partial J(t)}{\partial \omega_{i j}(t)}=\omega_{i j}(t)-\eta \frac{\partial E_{p}(t)}{\partial \omega_{i j}(t)}
$$

In equation (3.7), $\eta$ is step length. Here

$$
\frac{\partial E_{p}}{\partial \omega_{i j}}=\frac{\partial E_{p}}{\partial x_{i p}} \cdot \frac{\partial x_{i p}}{\partial \omega_{i j}}
$$

Let

$$
\delta_{i p}=\frac{\partial E_{p}}{\partial x_{i p}}
$$

Based on equation (3.8) and (3.9),

$$
\frac{\partial E_{p}}{\partial \omega_{i j}}=\delta_{i p} I_{j p}
$$

There have two conditions about $\delta_{i p}$,

If $i$ is output node of the neural network, means $i=k$,

$$
\delta_{i p}=\delta_{k p}=\frac{\partial E_{p}}{\partial x_{k p}}=\frac{\partial E_{p}}{\partial y_{k p}} \frac{\partial y_{k p}}{\partial x_{k p}}=-e_{k p} f^{\prime}\left(x_{k p}\right)
$$


Use equation (3.10) substituted to equation (3.8), then,

$$
\frac{\partial E_{p}}{\partial \omega_{i j}}=-e_{k p} f^{\prime}\left(x_{k p}\right) I_{j p}
$$

if $i$ is not an output node of neural networks, mean $i \neq k$,

$$
\delta_{i p}=\frac{\partial E_{p}}{\partial x_{i p}}=\frac{\partial E_{p}}{\partial y_{i p}} \cdot \frac{\partial y_{i p}}{\partial x_{i p}}=\frac{\partial E_{p}}{\partial y_{i p}} \cdot f^{\prime}\left(x_{k p}\right)
$$

among

$$
\begin{aligned}
\frac{\partial E_{p}}{\partial y_{i p}}= & \sum_{m_{1}} \frac{\partial E_{p}}{\partial x_{m_{1} p}} \cdot \frac{\partial x_{m_{1} p}}{\partial y_{i p}}=\sum_{m_{1}} \frac{\partial E_{p}}{\partial x_{m_{1} p}} \cdot \frac{\partial}{\partial y_{i p}} \sum_{j} \omega_{m_{1} j} I_{j p}^{*} \\
& =\sum_{\mathrm{m}_{1}} \frac{\partial E_{p}}{\partial \mathrm{x}_{\mathrm{m}_{1} \mathrm{p}}} \omega_{\mathrm{m}_{1} \mathrm{i}}=\sum_{\mathrm{m}_{1}} \delta_{\mathrm{m}_{1} \mathrm{p}} \omega_{\mathrm{m}_{1} \mathrm{i}}
\end{aligned}
$$

In equation (3.14), $m_{1}$ is a node that in the layer which following the layer include $i, I_{j p}^{*}$ is the $j t h$ input of node $m_{1}$.

Use equation (3.13) and (3.14) substitute to equation (3.8), the weight adjust function can be got,

$$
\frac{\partial E_{p}}{\partial \omega_{i j}}=f^{\prime}\left(x_{k p}\right) I_{j p}^{*} \sum_{\mathrm{m}_{1}} \frac{\partial \mathrm{E}^{\mathrm{p}}}{\partial \mathrm{x}_{\mathrm{m}_{1} \mathrm{p}}} \omega_{\mathrm{m}_{1} \mathrm{i}}=f^{\prime}\left(x_{k p}\right) I_{j p}^{*} \sum_{\mathrm{m}_{1}} \delta_{\mathrm{m}_{1} \mathrm{p}} \omega_{\mathrm{m}_{1} \mathrm{i}}
$$

Now, based on equation (3.15) and (3.12), the weight can be adjusted. 


\section{EXPERIMENT IMPLEMENTATION}

\subsection{Experimental Battery Characteristic}

The high power cell 18650-HE4 lithium-ion batteries are utilized in the charge/discharge experiments. Figure 4-2 shows battery discharge experimental equipment. The lithium-ion battery has a nominal capacity $2.5 \mathrm{Ah}$. The nominal voltage is $3.7 \mathrm{~V}$, setting the maximum charging voltage to $4.2 \mathrm{~V}$ and the cutoff voltage to $2.5 \mathrm{~V}$. Figure $4-1$ shows the thermal chamber and the measurement equipment, the test is carried out under $25.3^{\circ} \mathrm{C}$ in a constant thermal regulated chamber to reduce the influence of temperature. Thus, the battery's temperature varies within a very small range during the charge-discharge cycles. In this experiment, the temperature variation is not considered, and record the battery's terminal voltage, current, and the SOC states every five seconds.

In order to eliminate the measurement errors and environmental noise, run the identical experiments with several lithium-ion batteries. Figure 4-3 shows the example of five cells terminal voltage curves.

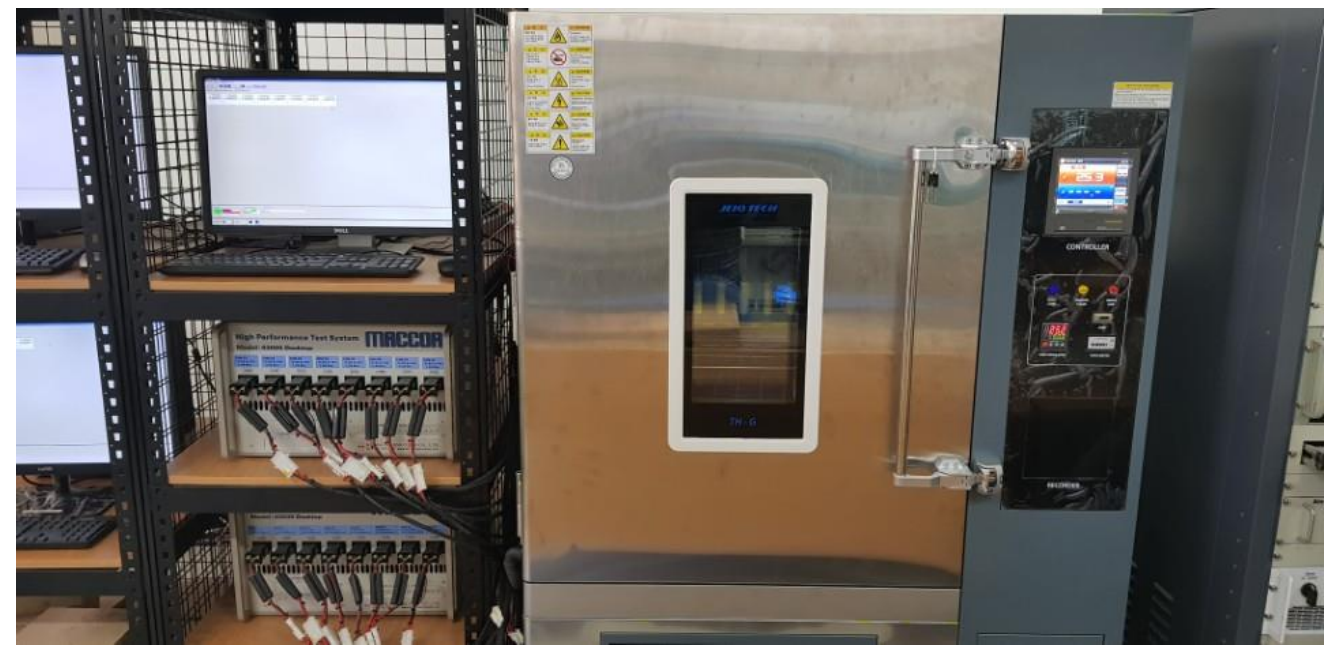

Figure 4-1 Thermal chamber and measurement equipment 


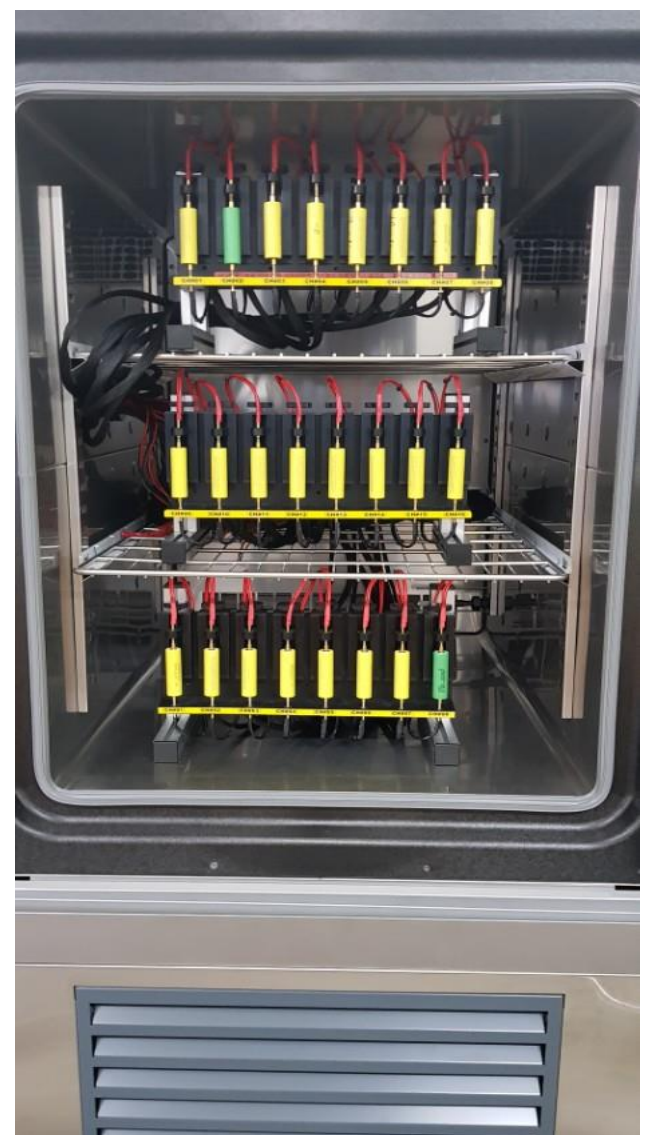

Figure 4-2 Battery discharging bench

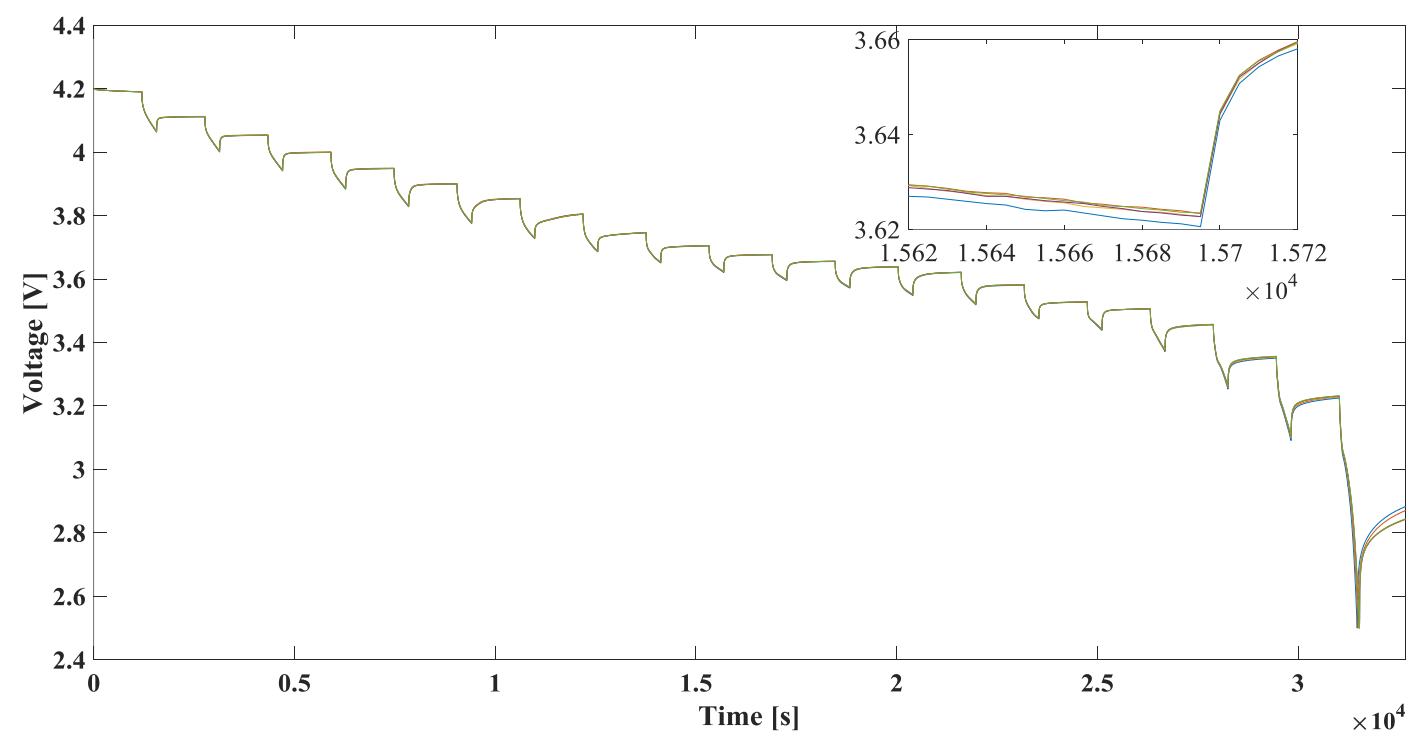

Figure 4-3 Experimental voltages of five batteries 


\subsection{Experimental Data Preprocessing}

There are plenty of clustering algorithms for the experimental data. In this paper, K-means algorithm is chosen because it is easy to implement and effective.

$\mathrm{K}$-means clustering is a form of unsupervised learning algorithm; it can partition the data to clusters of patterns in such a way that the measure of similarity with the set of data assigned to central point minimizing a specified cost function [28].

Let $X_{m \times n}$ denote a set of multidimensional experimental data. Each column represents a battery's terminal voltage. The $X_{m \times n}$ can be partitioned into the $\mathrm{K}$ clusters, and usually $\mathrm{K}$ is smaller than the number of rows, $m$

Assuming

$$
j=C(i)
$$

Where $i=1,2, \cdots m$.

Equation (4.1) is so-called mapper or encoder. It can assign the $i$ th $X_{i}$ to the $j$ th cluster based on the specified cost function. Considering the two data sets $X_{i}$ and $X_{i^{*}}$, assigning $d\left(X_{i}, X_{i^{*}}\right)$ to denote the similarity between vector $X_{i}$ and $X_{i^{*}}$. The $X_{i}$ and $X_{i^{*}}$ belong to the same clustering when the distance between $X_{i}$ and $X_{i^{*}}$ is small enough.

In 2001, Hastie proposed the cost function [29]:

$$
J(C)=\frac{1}{2} \sum_{j=1}^{k} \sum_{C(i)=j} \sum_{C\left(i^{*}\right)=j} d\left(X_{i}-X_{i^{*}}\right)
$$

For a specific $\mathrm{K}$, the goal of the cost function is trying to find the mapper which can make the cost function $J(C)$ is minimized.

In K-means algorithm, the squared Euclidean norm is assigned as the function to measure the similarity between the experimental data $X_{i}$ and $X_{i^{*}}$. The similarity function defined 


$$
d\left(X_{i}, X_{i^{*}}\right)=\left\|X_{i}-X_{i^{*}}\right\|^{2}
$$

Substituting equation (4.3) to equation (4.2), then,

$$
J(C)=\frac{1}{2} \sum_{j=1}^{k} \sum_{C(i)=j} \sum_{C\left(i^{i}\right)=j}\left\|X_{i}-X_{i^{*}}\right\|^{2}
$$

Based on the equation (4.4), use five sets of experimental terminal voltage as observations, use K-means to eliminate the measurement errors.

Table 4-1 shows the example that K-means processed data, and Table 4-2 shows that error between K-means value and experimental data. K-means reduce the measurement error. The more experimental data used as observations, the better result that K-means can get.

Table 4-1 The battery's voltage and K-means clustering voltage

\begin{tabular}{c|c|c|c|c|c}
\hline $\begin{array}{c}\text { Battery } \\
1\end{array}$ & $\begin{array}{c}\text { Battery } \\
2\end{array}$ & $\begin{array}{c}\text { Battery } \\
3\end{array}$ & $\begin{array}{c}\text { Battery } \\
4\end{array}$ & $\begin{array}{c}\text { Battery } \\
5\end{array}$ & K-means \\
\hline 4.1984 & 4.1994 & 4.1994 & 4.1991 & 4.1992 & 4.1991 \\
\hline 4.1986 & 4.1987 & 4.1987 & 4.1984 & 4.1989 & 4.1987 \\
\hline 4.1983 & 4.1984 & 4.1984 & 4.1986 & 4.1987 & 4.1985 \\
\hline 4.1980 & 4.1984 & 4.1981 & 4.1980 & 4.1984 & 4.1982 \\
\hline 4.1978 & 4.1981 & 4.1981 & 4.1981 & 4.1984 & 4.1982 \\
\hline
\end{tabular}

Table 4-2 K-means error

\begin{tabular}{l|l}
\hline Times & K-means error \\
\hline 1 & $5.774 \times 10^{-7}$ \\
\hline 2 & $1.210 \times 10^{-7}$ \\
\hline 3 & $1.210 \times 10^{-7}$ \\
\hline 4 & $2.142 \times 10^{-7}$ \\
\hline 5 & $1.862 \times 10^{-7}$ \\
\hline
\end{tabular}

Figure 4-4 shows the curve of the battery terminal voltage. The voltage data that K-means processed is used to run the battery's model parameters identification. 


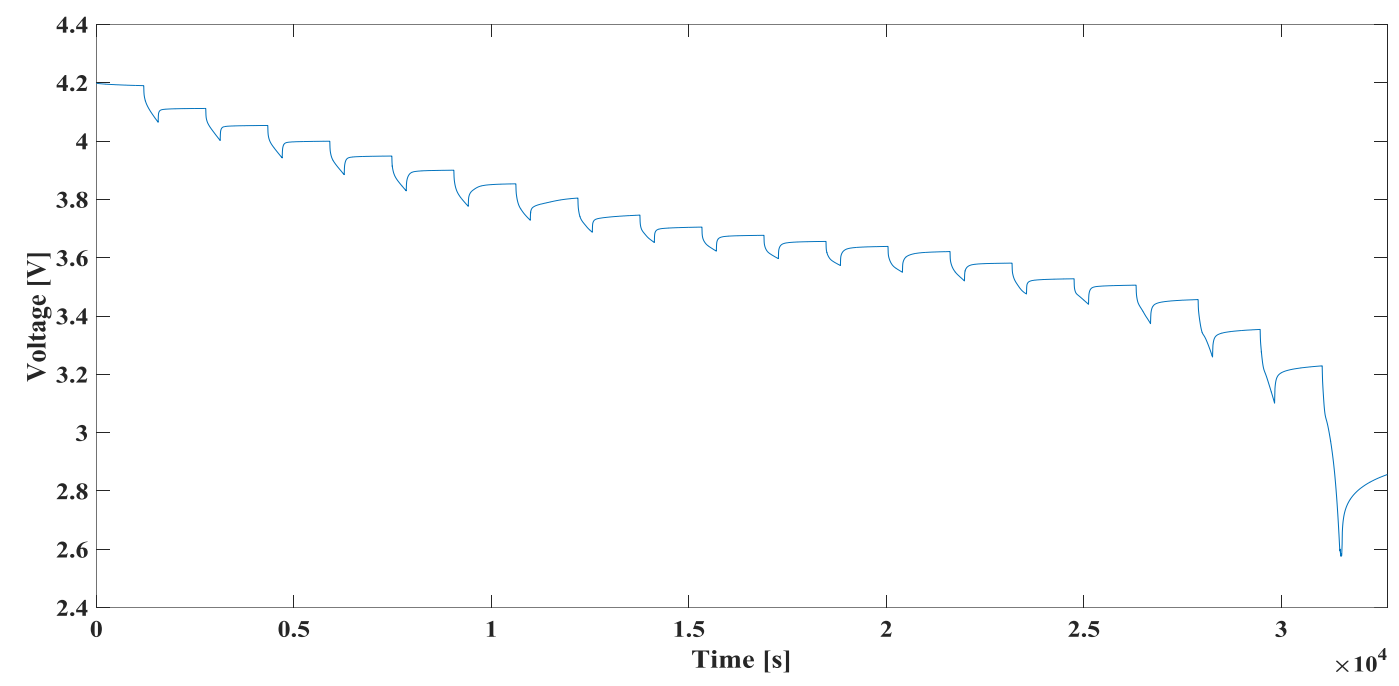

Figure 4-4 Experimental data of the battery's terminal voltage

Figure 4-5 shows the curve of battery's SOC versus terminal voltage. The curve of figure 4-3 is not like traditional OCV-SOC curve because of two reasons. First, the terminal voltage is not always close to OCV because after a long charge/discharge operation, the polarization effect has an influence on the battery's performance. Secondly, in this experiment the battery is rest under open circuit conditions after 5\% SOC discharge. In this period, the battery's voltage varies to the equilibrium state. Usually, the voltage is increased in the period.

Figure 4-6 shows the current response corresponding terminal voltage. In the experiment, the battery is charged with the half rated current until reach the maximum charging voltage $4.2 \mathrm{~V}$, then charge with a trickle-current until it is totally charged.

As shown in figure 4-6. The current is not precise constant value $1.25 \mathrm{~A}$ due to the small supplying power variation. 


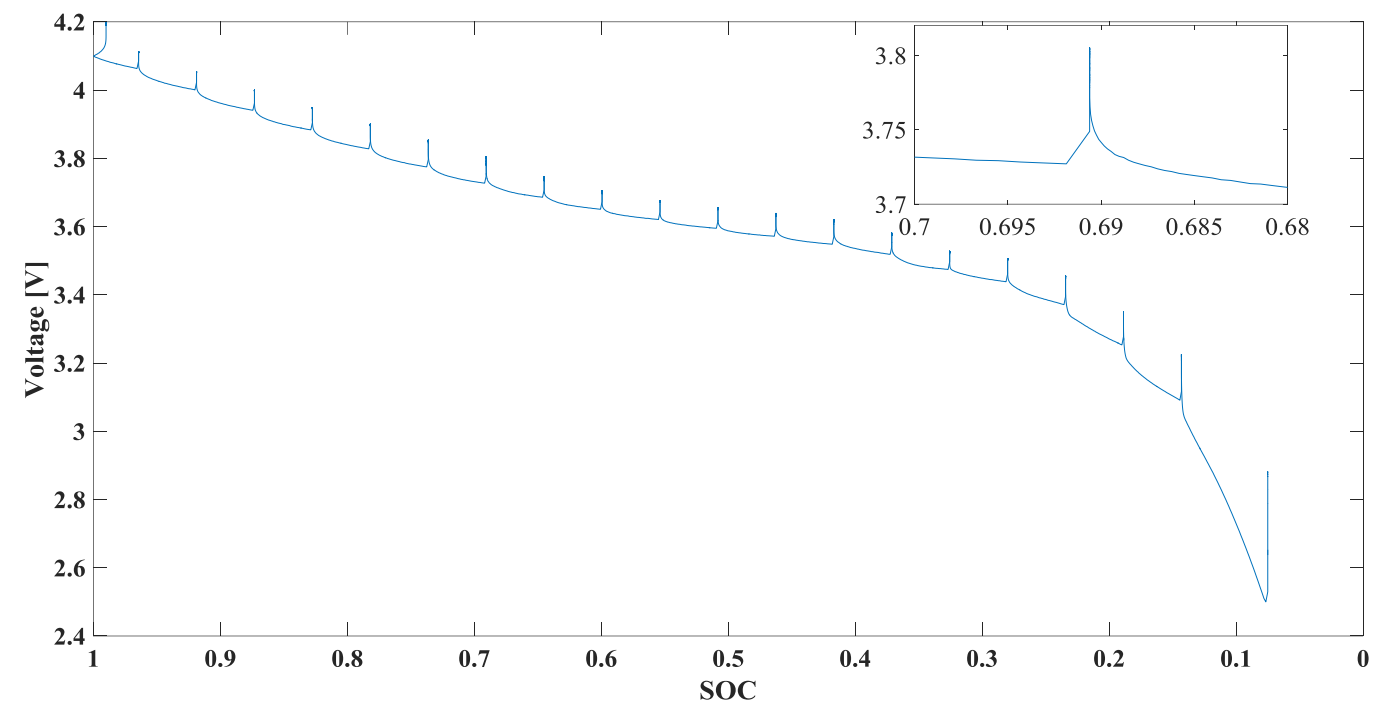

Figure 4-5 The SOC versus terminal voltage

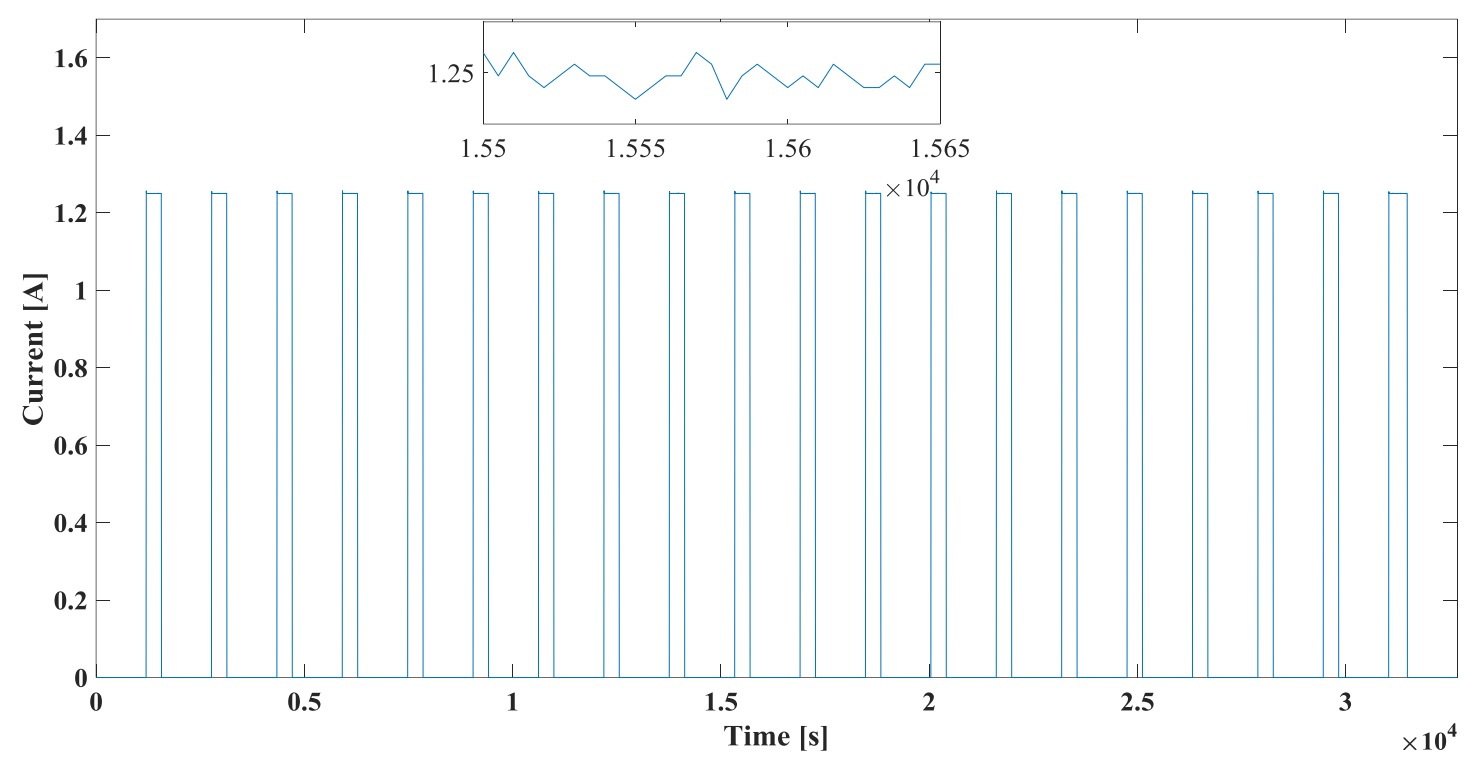

Figure 4-6 Current of the load 
To acquire data to identify the parameters of the battery model, a discharge test was performed on the 18650-HE4 lithium-ion battery. The battery is at rest more than 20 minutes to reach the equilibrium state after the full charge. In the discharge experiment, the battery is first discharged at a $0.5 \mathrm{C}$ rated current from the fully charged state to $95 \%$ of the nominal capacity. Afterward, the battery is left 20 minutes under open circuit condition. The operation is repeated until the SOC turns to zero or the battery is fully discharge.

Figure 4-8 shows the two curves that represent OCV versus SOC, and the terminal voltage versus SOC. The experiment is run under the same conditions and use the same battery. Figure 4-7 shows the curve of SOC versus time. There are twenty sample points in the figure. The OCV-SOC curve uses the data from an experiment that discharge at $1.25 \mathrm{~A}$ current in steps of $5 \%$ of rated battery capacity, taking 20minutes rest at each discharge cycle and measuring the voltage. The terminal voltage versus SOC is obtained from the experiment with discharging at $1.25 \mathrm{~A}$ current continuously.

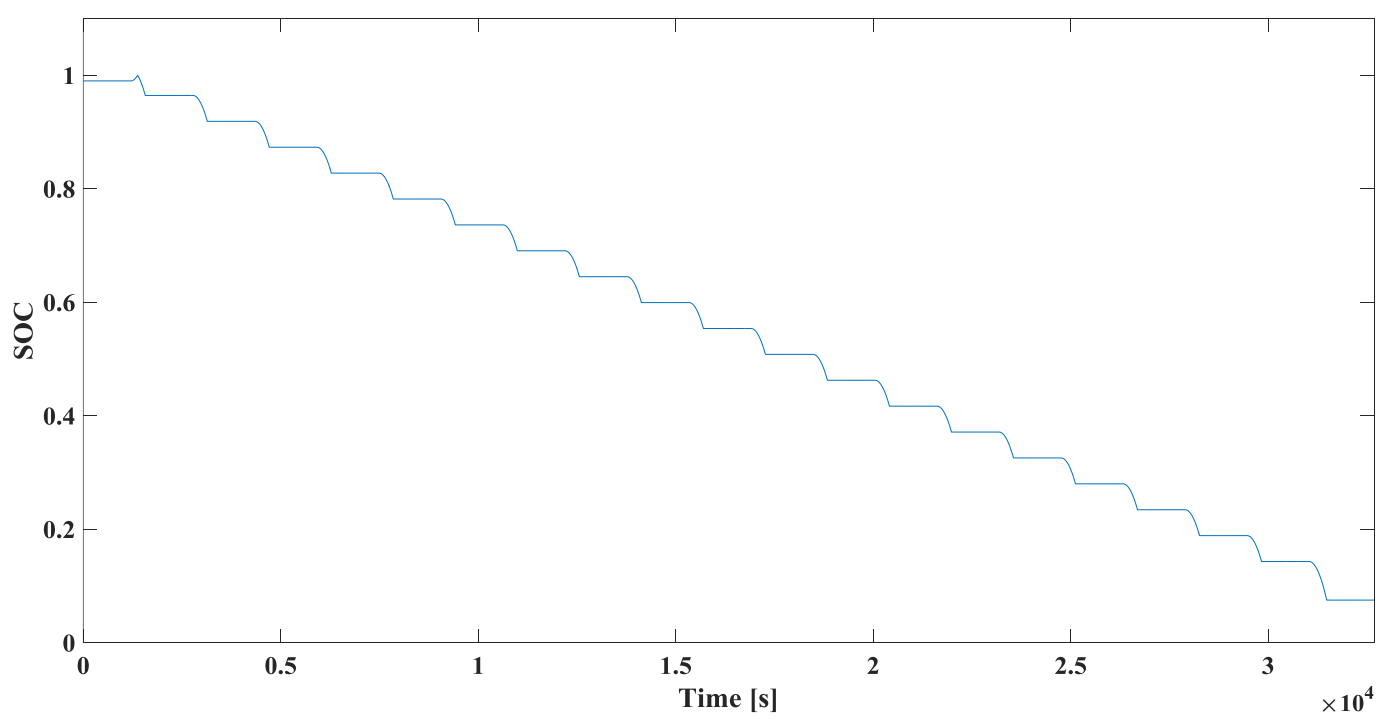

Figure 4-7 The experimental SOC curve 


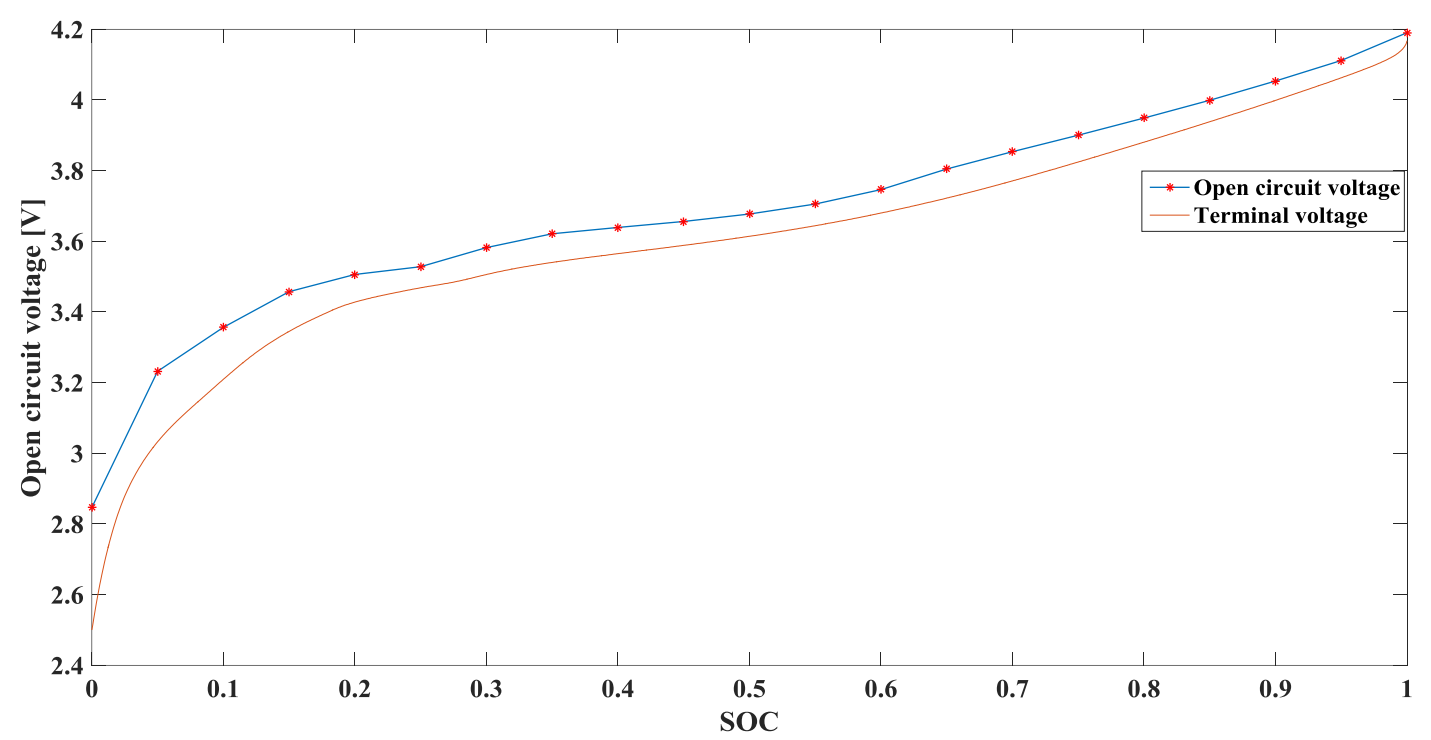

Figure 4-8 SOC versus OCV and terminal voltage

Table 4-3 lists the experiment data SOC and the open circuit voltage, the SOC is not discharged to $0 \%$ SOC. During the discharge process, the terminal voltage is reduced to $2.499961852445 \mathrm{~V}$, which is lower than the cutoff voltage $2.5 \mathrm{~V}$. In order to avoid over discharging causing the permanent internal damage for the battery, the battery is set to be at rest. Avoiding overcharging or discharging is the main study area in BMS, in this paper, the damage that is caused by over discharging is out of scope of the study. The battery is discharged in steps of $5 \%$ of the rated capacity.

Usually, the terminal voltage is a function of temperature, SOC, and discharge duration time. The measured terminal voltage is considered as the open circuit voltage or equilibrium potential every 20 -minutes interval. Also, the equilibrium is higher than the terminal voltage at discharging process. 
Table 4-3 OCV and SOC measurement value

\begin{tabular}{l|l}
\hline SOC $(\%)$ & Open circuit voltage $(\mathrm{V})$ \\
\hline 100 & 4.190356298161 \\
\hline 95.44 & 4.111772335393 \\
\hline 90.87 & 4.053635461967 \\
\hline 86.31 & 3.999160753796 \\
\hline 81.74 & 3.948805981537 \\
\hline 77.18 & 3.900282291905 \\
\hline 72.61 & 3.853589684901 \\
\hline 68.05 & 3.804608224613 \\
\hline 63.48 & 3.746318760967 \\
\hline 58.92 & 3.705424582284 \\
\hline 54.36 & 3.677195391775 \\
\hline 49.80 & 3.656137941558 \\
\hline 45.23 & 3.639200427253 \\
\hline 40.67 & 3.621347371633 \\
\hline 36.11 & 3.582131685359 \\
\hline 31.54 & 3.528114747845 \\
\hline 26.98 & 3.506141756313 \\
\hline 22.42 & 3.457465476463 \\
\hline 17.85 & 3.356450751507 \\
\hline 13.29 & 3.232089723049 \\
\hline 4.70 & 2.8463416495 \\
\hline & \\
\hline
\end{tabular}




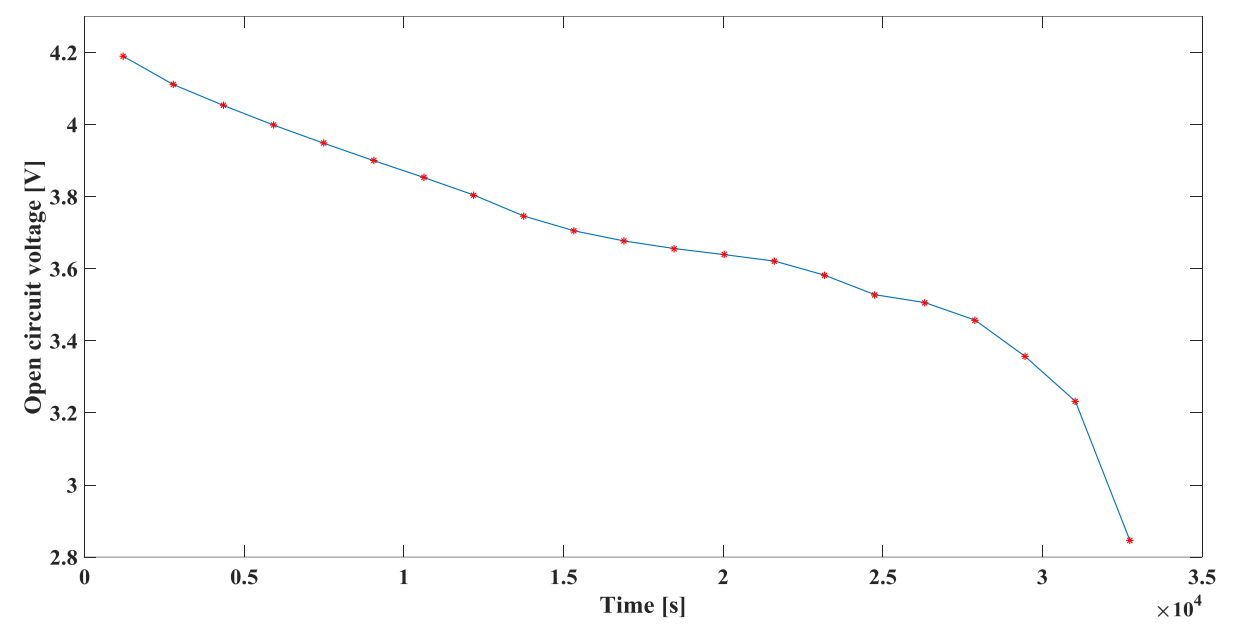

Figure 4-9 Open circuit voltage curve

Figure 4-9 shows the OCV versus time curve, the OCV of battery drop during the discharge operation in a nonlinear way. 


\section{EXPERIMENT RESULTS AND ANALYSIS}

\section{$\underline{5.1 \text { Battery Model Identification }}$}

Increasing the battery model order can improve the estimation accuracy because the battery performs different dynamic characteristics during different charge/discharge periods. However, the high order battery requires more computation resources.

The electrical behavior of the Thevenin model is represented as follows.

$$
\begin{gathered}
C_{p} \frac{d U_{p}(t)}{d t}=-\frac{U_{p}(t)}{R_{p}}+i(t) \\
U(t)=U_{o c}(t)-R_{o} i(t)-U_{p}(t)
\end{gathered}
$$

Where,

$i(t) \quad$ is the load current

$U_{p}(t) \quad$ is polarization voltage

$U(t) \quad$ is terminal voltage

By defining $U_{q}(t)=U(t)-U_{p}(t)$, the equation (5.1) can be expressed as equation (5.3)

$$
\begin{gathered}
\frac{U_{q}(s)}{i(s)}=\frac{-\left(R_{o}+R_{p}+R_{o} R_{p} C_{p} s\right)}{1+R_{p} C_{p} s} \\
s=\frac{2}{T} \cdot \frac{1-q^{-1}}{1+q^{-1}}
\end{gathered}
$$

Applying the bilinear transform equation (5.4) to equation (5.3) yields

$$
\frac{U_{q}\left(q^{-1}\right)}{i\left(q^{-1}\right)}=\frac{a+b q^{-1}}{1+d q^{-1}}
$$


By substituting equation (5.4) into equation (5.3), the frequency domain can be reversed to the discrete time domain, we have

$$
\left[\begin{array}{l}
a \\
b \\
c
\end{array}\right]=\left[\begin{array}{l}
\left(T-2 R_{p} C_{p}\right) /\left(T+2 R_{p} C_{p}\right) \\
-\left(R_{o} T+R_{p} T+2 R_{o} R_{p} C_{p}\right) /\left(T+2 R_{p} C_{p}\right) \\
-\left(R_{o} T+R_{p} T-2 R_{o} R_{p} C_{p}\right) /\left(T+2 R_{p} C_{p}\right)
\end{array}\right]
$$

Based on equation (5.6), the parameters of Thevenin model of battery are

$$
\left[\begin{array}{l}
R_{o} \\
R_{p} \\
C_{p}
\end{array}\right]=\left[\begin{array}{l}
\frac{c-b}{1-a} \\
\frac{2(a b-c)}{1-a^{2}} \\
\frac{(1-a)^{2} T}{4(a b-c)}
\end{array}\right]
$$

The mathematical expression of the improved Thevenin model is

$$
U_{o c}=\left(\frac{R_{p}}{R_{p} C_{p} s+1}+\frac{R_{c}}{R_{c} C_{c} s+1}+R_{0}\right) I+U
$$

Let

$$
\begin{gathered}
\tau_{1}=R_{p} C_{p} \\
\tau_{2}=R_{c} C_{c}
\end{gathered}
$$

By substituting equation (5.9) and (5.10) into equation (5.8)

$$
\begin{aligned}
\tau_{1} \tau_{2} U_{o c} s^{2}+\left(\tau_{1}+\tau_{2}\right) U_{o c} s & +U_{o c}=\tau_{1} \tau_{2} R_{0} I s^{2}+\left[\tau_{2} R_{p}+\tau_{1} R_{c}+R_{0}\left(\tau_{1}+\tau_{2}\right)\right] I s \\
& +\left(R_{0}+R_{p}+R_{c}\right) I+\tau_{1} \tau_{2} U s^{2}+\left(\tau_{1}+\tau_{2}\right) U s+U
\end{aligned}
$$

Let

$$
a=\tau_{1} \tau_{2}
$$




$$
\begin{gathered}
b=\tau_{1}+\tau_{2} \\
c=R_{0}+R_{p}+R_{c} \\
d=\tau_{2} R_{p}+\tau_{1} R_{c}+R_{0}\left(\tau_{1}+\tau_{2}\right)
\end{gathered}
$$

Then

$$
a U_{o c} s^{2}+b U_{o c} s+U_{o c}=a R_{0} I s^{2}+d I s+c I+a U s^{2}+b U s+U
$$

Let

$$
\left\{\begin{array}{l}
s^{2}=\frac{[x(k)-2 x(k-1)+x(k-2)]}{T^{2}} \\
s=\frac{[x(k)-x(k-1)]}{T}
\end{array}\right.
$$

By substituting discrete-time equation (5.17) into equation (5.16) yields discrete-time,

$$
\begin{aligned}
U_{o c}(k)-U(k)= & \frac{-b T-2 a}{T^{2}+b T+a}\left[U(k-1)-U_{o c}(k-1)\right] \\
& +\frac{a}{T^{2}+b T+a}\left[U(k-2)-U_{o c}(k-2)\right] \\
& +\frac{c T^{2}+d T+a R_{0}}{T^{2}+b T+a} I(k)+\frac{-d T+2 a R_{0}}{T^{2}+b T+a} I(k-1) \\
& +\frac{a R_{0}}{T^{2}+b T+a} I(k-2)
\end{aligned}
$$

Let 


$$
\left\{\begin{array}{l}
K_{1}=\frac{-b T-2 a}{T^{2}+b T+a} \\
K_{2}=\frac{a}{T^{2}+b T+a} \\
K_{3}=\frac{c T^{2}+d T+a R_{0}}{T^{2}+b T+a} \\
K_{4}=\frac{-d T+2 a R_{0}}{T^{2}+b T+a} \\
K_{5}=\frac{a R_{0}}{T^{2}+b T+a}
\end{array}\right.
$$

Substituting equation (5.19) into equation (5.18) yields

$$
\begin{aligned}
U_{o c}(k)-U(k)= & K_{1}\left[U(k-1)-U_{o c}(k-1)\right] \\
& +K_{2}\left[U(k-2)-U_{o c}(k-2)\right] \\
& +K_{3} I(k)+K_{4} I(k-1) \\
& +K_{5} I(k-2)
\end{aligned}
$$

Let $K_{0}=T^{2}+b T+a$, then the relationship between coefficients can be got

$$
\left\{\begin{array}{l}
K_{0}=\frac{T^{2}}{K_{1}+K_{2}+1} \\
a=K_{0} \square K_{2} \\
b=\frac{-K_{0}\left(K_{1}+2 K_{2}\right)}{T} \\
c=\frac{K_{0}\left(K_{3}+K_{4}+K_{5}\right)}{T^{2}} \\
d=\frac{-K_{0}\left(K_{4}+K_{5}\right)}{T} \\
R_{0}=\frac{K_{5}}{K_{2}}
\end{array}\right.
$$

Based on equation (5.12) and (5.13), we get 


$$
\left\{\begin{array}{l}
R_{1}=c-R_{c}-R_{0} \\
R_{2}=\frac{c \tau_{2}+R_{0} b-\tau_{2} R_{0}-d}{\tau_{2}-\tau_{1}} \\
\tau_{1}=\min \left(\frac{b+\sqrt{b^{2}-4 a}}{2}, \frac{b-\sqrt{b^{2}-4 a}}{2}\right) \\
\tau_{2}=\max \left(\frac{b+\sqrt{b^{2}-4 a}}{2}, \frac{b-\sqrt{b^{2}-4 a}}{2}\right) \\
C_{p}=\frac{\tau_{1}}{R_{p}} \\
C_{c}=\frac{\tau_{2}}{R_{c}}
\end{array}\right.
$$

Where the solutions of ${ }^{\tau_{1}}$ and ${ }^{\tau_{2}}$ are based on equation

$$
\tau^{2}-b \tau+a=0
$$

\subsection{Experiment for Parameter Extraction}

Figure 5-1 shows the terminal voltage response during the rest interval with $\mathrm{SOC}=80 \%$ and $\mathrm{SOC}=75 \%$. The OCV of SOC $=80 \%$ is the terminal voltage of the battery at the end of rest interval. The ohmic resistance $R_{0}$ at the corresponding SOCs can be given by

$$
R_{0}=\frac{U}{I}
$$

The parameters of the improved Thevenin model at each SOC can be determined by the terminal voltage response at corresponding SOCs.

$$
\begin{gathered}
U=U_{p}\left(1-e^{-\frac{t}{\tau_{p}}}\right)+U_{f}\left(1-e^{-\frac{t}{\tau_{f}}}\right)+U_{i} \\
U_{p}=R_{p} I
\end{gathered}
$$




$$
\begin{gathered}
U_{c}=R_{c} I=\left[\frac{\left(U_{o c}(t)-U_{i}\right)}{I}-R_{p}\right] \cdot I \\
\tau_{p}=R_{p} C_{p} \tau_{p}=R_{c} C_{c}
\end{gathered}
$$

Where

$I \quad$ is battery's real-time current before the rest period

$U_{p} \quad$ is the short-term transient voltage

$U_{c} \quad$ is the long-term transient voltage

$\tau_{p} \quad$ is the time constant corresponding to the $U_{p}$

$\tau_{c} \quad$ is the time constant corresponding to the $U_{c}$

$U_{o c}(t)$ is the OCV at the corresponding SOC

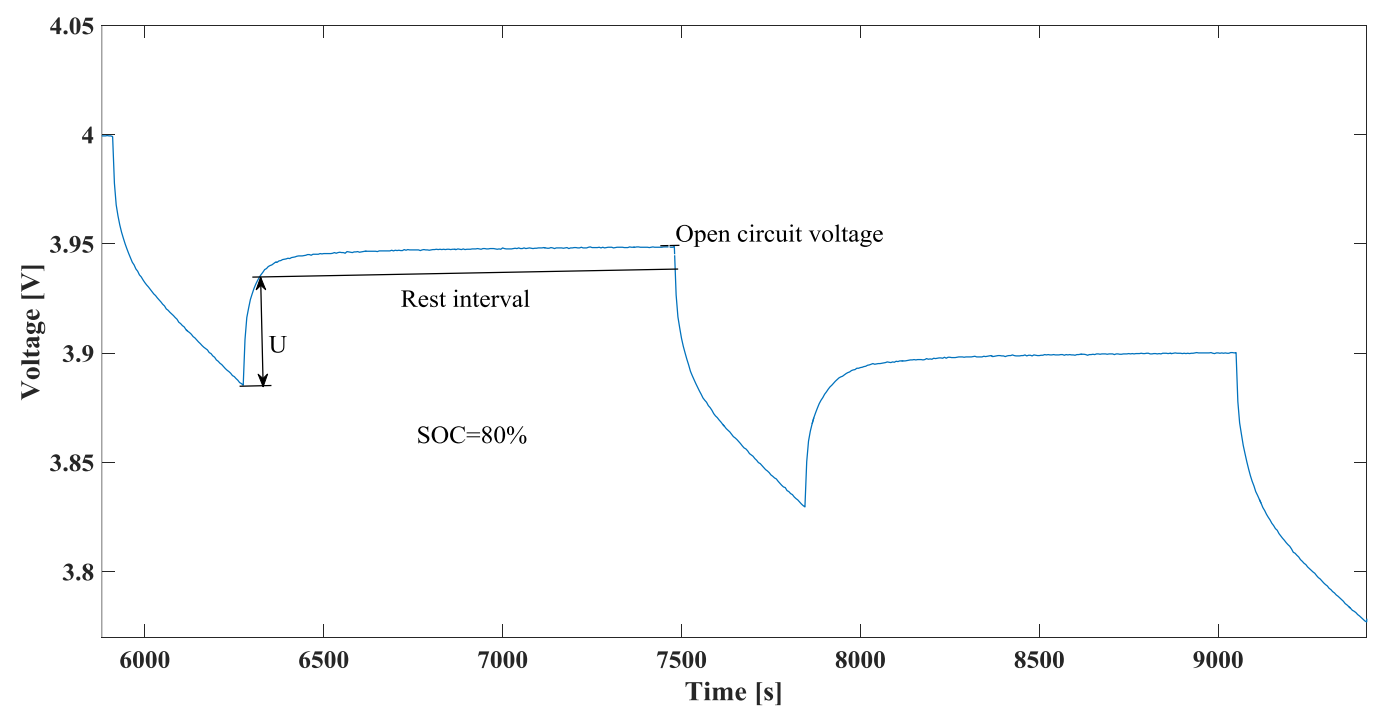

Figure 5-1 Battery's terminal voltage response

Figures 5-2 to 5-6 show the parameters identification results at the twenty different SOCs, the red dots are the experimental data points. The identification model is improved Thevenin model. 


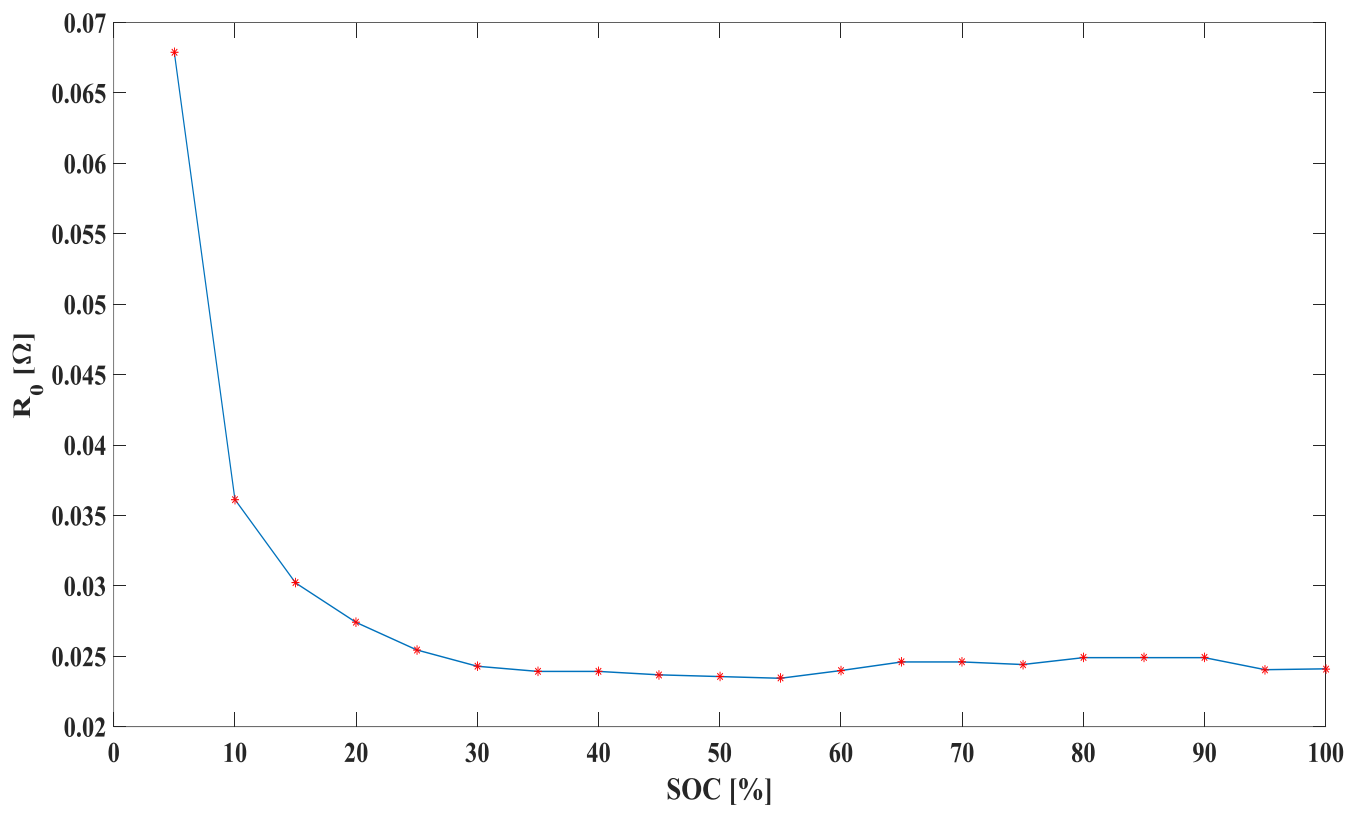

Figure 5-2 Ohiom resistance curve

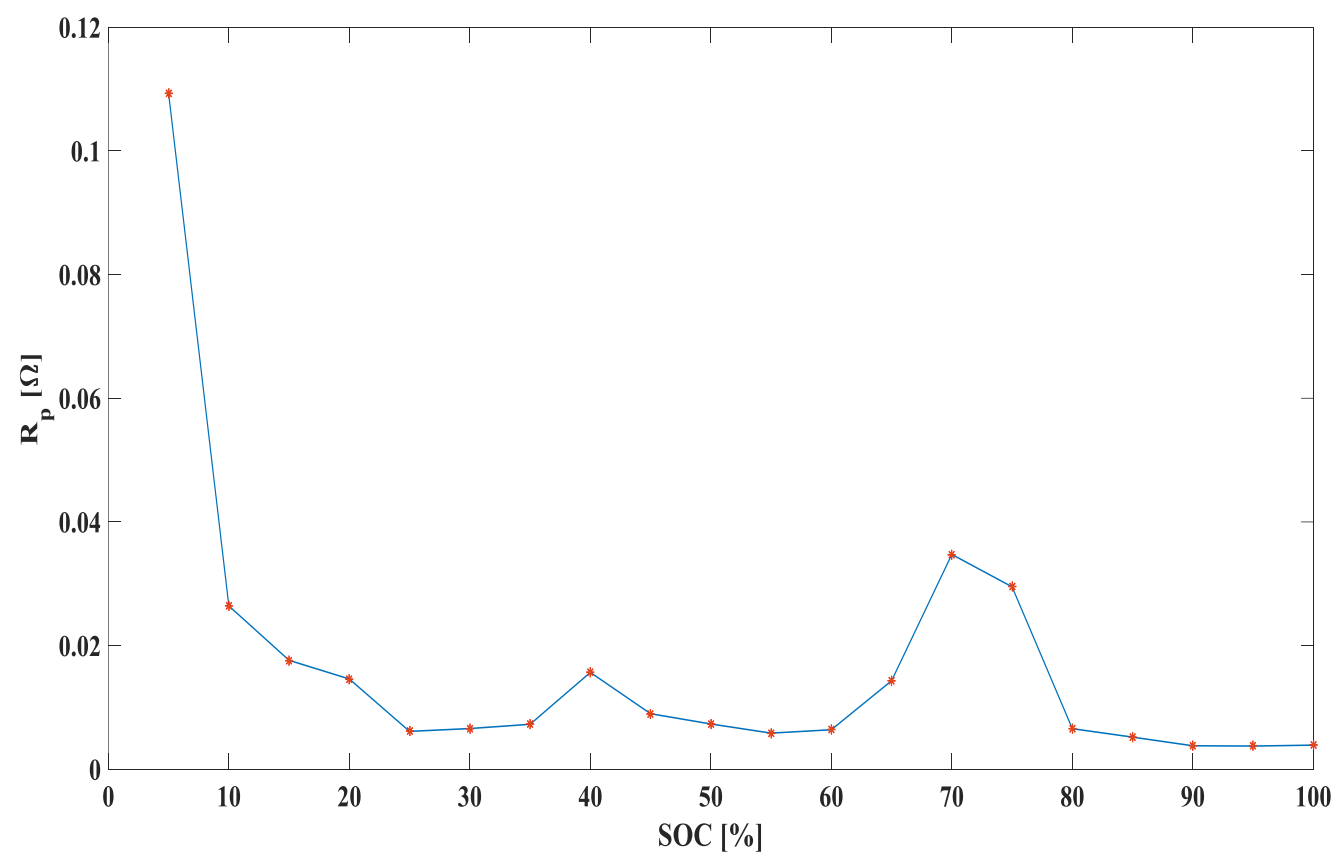

Figure 5-3 Curve of $R_{p}$ 


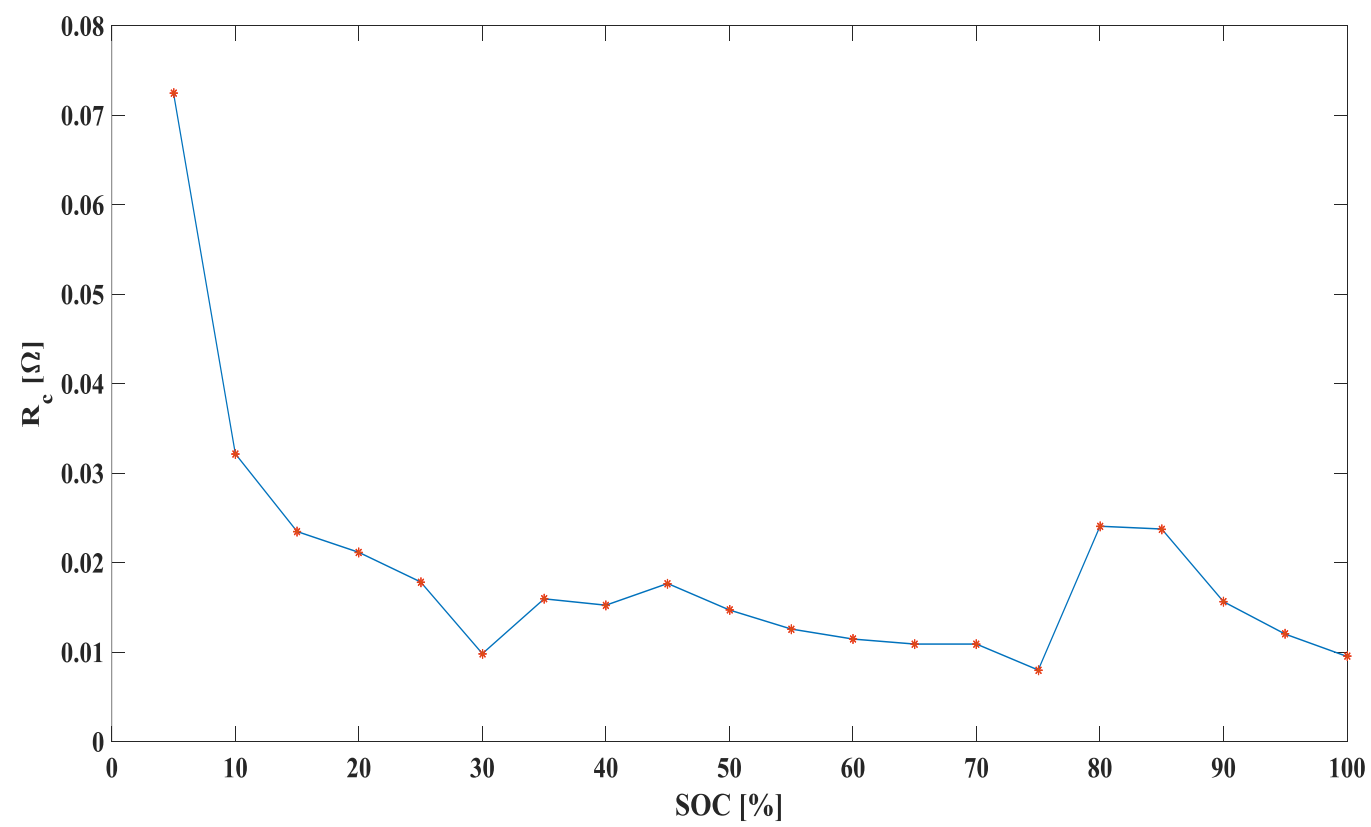

Figure 5-4 Curve of $R_{c}$

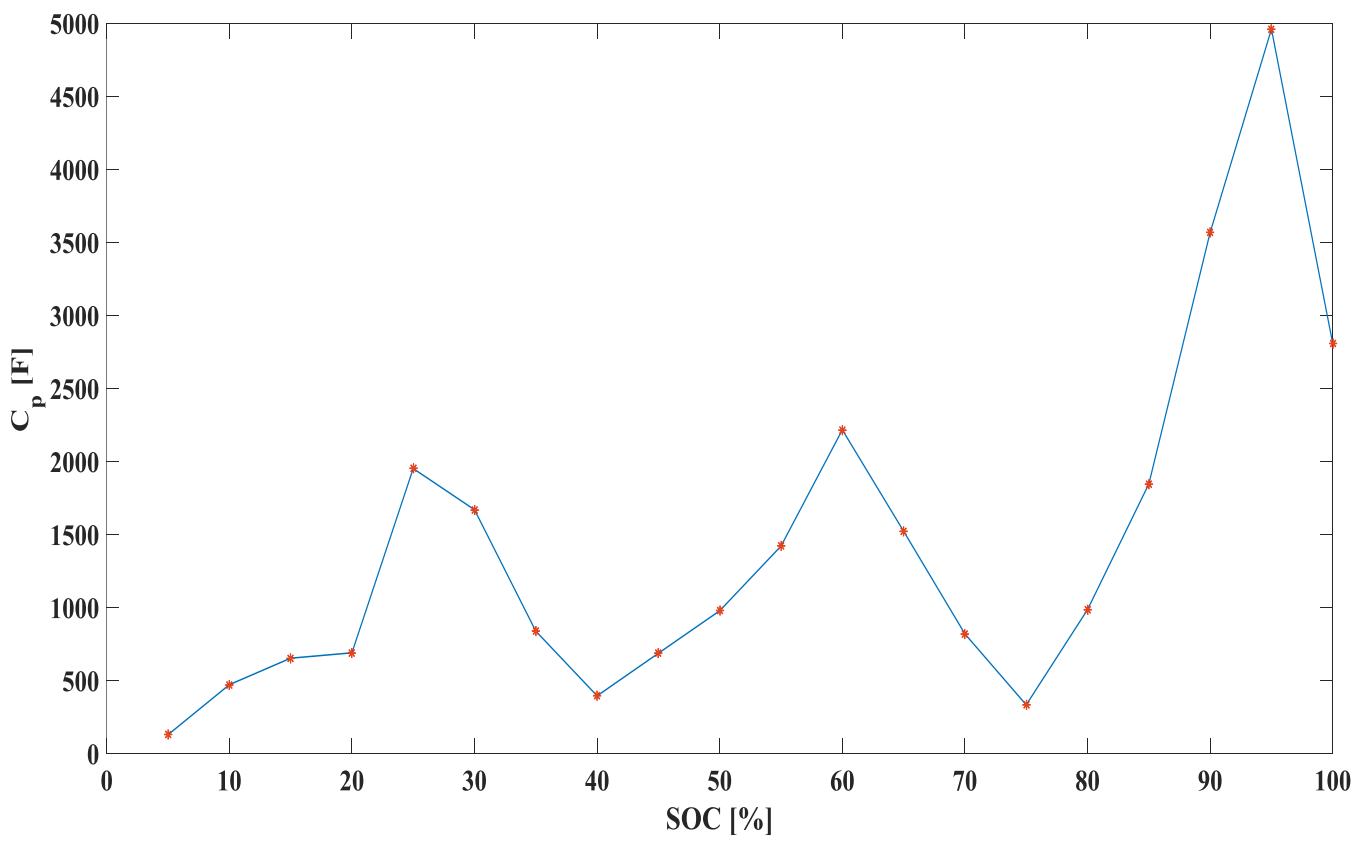

Figure 5-5 Curve of $C_{p}$ 


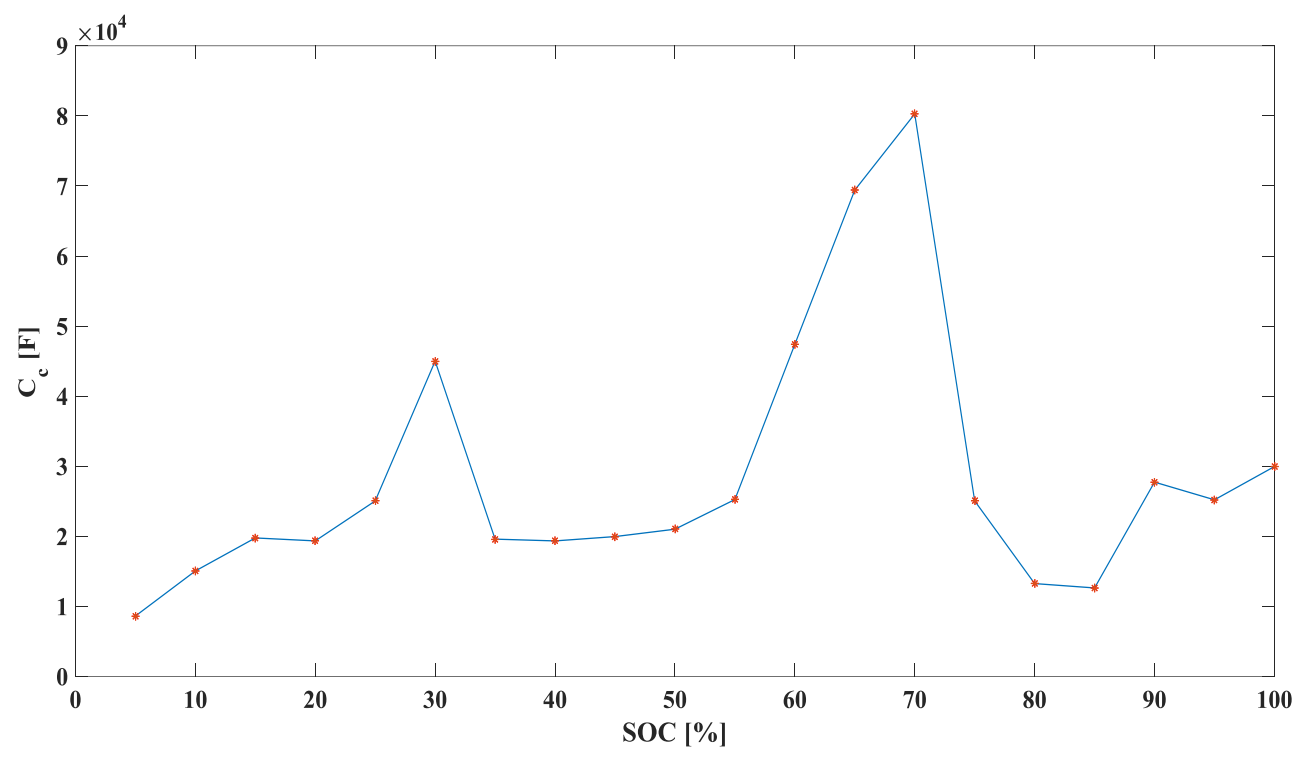

Figure 5-6 Curve of $C_{c}$

\subsection{Thevenin Model Identification Based on Neural Network}

The parameters identification for the Thevenin model and the improved model at corresponding SOCs is demonstrating that all the parameters of the model are varied [30]. However, using the exponential function to fit the experimental curve at corresponding points is not always practical, because this requires obtaining the OCV of the battery. That is impossible especially for the device that uses continuously.

To obtain more parameters information of the battery, the neural network is used, because neural networks still have very well performance because of its strong learning ability.

Figure 5-7 shows the structure of the neural network, where $\hat{U}(k) \quad$ is real-time terminal voltage estimation

$U_{o c}(k)$ is real-time $\mathrm{OCV}$

$w_{U(k-1)}, w_{I(k-1)} w_{I(k)}$ and $w_{U_{o c}(k)}$ are weight vectors. 


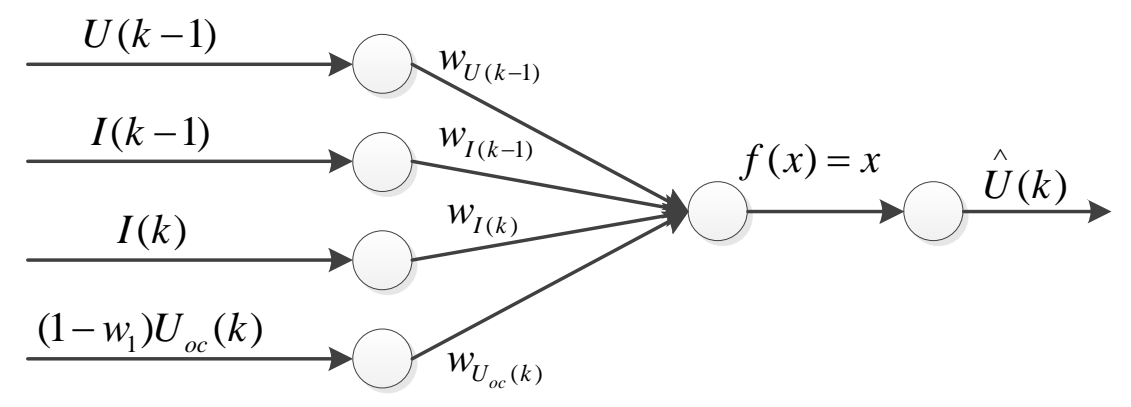

Input Hidden layer Output

Figure 5-7 BP neural network for the Thevenin model

The acting function used in this study is the linear function, or called as action function.

The initial values of the weights are carefully selected. if all initial values $w_{U(k-1)}, w_{I(k-1)}, w_{I(k)}$ and $w_{U_{o c}(k)}$ are same small values. Based on the knowledge of chapter 3, all the adjustment of weights of the hidden layer will be the same that may cause all weights are the same. Therefore, the weight should be set to random value.

The good step length of learning $\eta$ is important at the beginning of learning. The big $\eta$ can accelerate the learning speed, but the system will oscillate and cannot converge when system is close to the optimum point. Thus, the very small $\eta$ must be set when output close to desired values.

In order to compensate the slow speed of learning at the beginning, the inertia coefficient $\alpha$ was added, based on chapter 3 .

$$
\omega(t+1)=\omega(t)+\eta \delta \cdot f[\mathrm{x}(t)]+\alpha[\omega(t)-\omega(t-1)]
$$

The BP algorithm is considered to have converged when the absolute rate of change in the mean square error (MSE) is small enough in each epoch. 
Also adding inertia part could help system avoid the local minimum. The current adjustment $\omega(\mathrm{t})-\omega(\mathrm{t}-1)$ represents the sum of a weighted time series. For the time series to be convergent, the inertia constant must be restricted to the range $0<|\alpha|<1$.

When the $\partial E_{p} / \partial \omega_{i j}$ has the same algebraic sign on consecutive iterations, $\partial E_{p} / \partial \omega_{i j}$ grows in the magnitude, the weight $\omega(t)-\omega(t-1)$ is adjusted by a large amount.

When the $\partial E_{p} / \partial \omega_{i j}$ has opposite signs on consecutive iterations, $\partial E_{p} / \partial \omega_{i j}$ shrinks in the magnitude, consequently the weight $\omega(t)-\omega(t-1)$ is adjusted by a small amount. The inclusion of momentum in the BP algorithm has a stabilizing effect.

Figure 5-8 shows the flowchart of the network, based on this the weights of the iterative algorithm are

$$
\begin{gathered}
\Delta \omega_{\mathrm{U}(k-1)}=-\eta \frac{\partial E(k)}{\partial \omega_{\mathrm{U}(k-1)}}=\eta(U(K-1)-\hat{U}(K-1)) \hat{U}(K-1) \\
\Delta \omega_{I(k-1)}=-\eta \frac{\partial E(k)}{\partial \omega_{I(k-1)}}=\eta(U(K-1)-\hat{U}(K-1)) I(k-1) \\
\Delta \omega_{I(k)}=-\eta \frac{\partial E(k)}{\partial \omega_{I(k)}}=\eta(U(K-1)-\hat{U}(K-1)) I(k) \\
\Delta \omega_{\mathrm{U}_{o c}(k)}=-\eta \frac{\partial E(k)}{\partial \omega_{I(k-1)}}=\eta(U(K-1)-\hat{U}(K-1))
\end{gathered}
$$

Where $\eta \in(0,1)$ is learning rate, based on equation (5.30) to (5.33), the iterative formula of weighing

$$
\begin{gathered}
\omega_{\mathrm{U}(k-1)}(k)=\omega_{\mathrm{U}(k-1)}(k-1)+\Delta \omega_{\mathrm{U}(k-1)}+\alpha \cdot\left(\omega_{\mathrm{U}(k-1)}(k-1)-\omega_{\mathrm{U}(k-1)}(k-2)\right) \\
\omega_{I(k-1)}(k)=\omega_{I(k-1)}(k-1)+\Delta \omega_{I(k-1)}+\alpha\left(\omega_{I(k-1)}(k-1)-\omega_{I(k-1)}(k-2)\right)
\end{gathered}
$$




$$
\begin{gathered}
\omega_{I(k)}(k)=\omega_{I(k)}(k-1)+\Delta \omega_{I(k)}+\alpha\left(\omega_{I(k)}(k-1)-\omega_{I(k)}(k-2)\right) \\
\omega_{\mathrm{U}_{o c}(k)}(k)=\omega_{\mathrm{U}_{o c}(k)}(k-1)+\Delta \omega_{\mathrm{U}_{o c}(k)}+\alpha\left(\omega_{\mathrm{U}_{o c}(k)}(k-1)-\omega_{\mathrm{U}_{o c}(k)}(k-2)\right)
\end{gathered}
$$

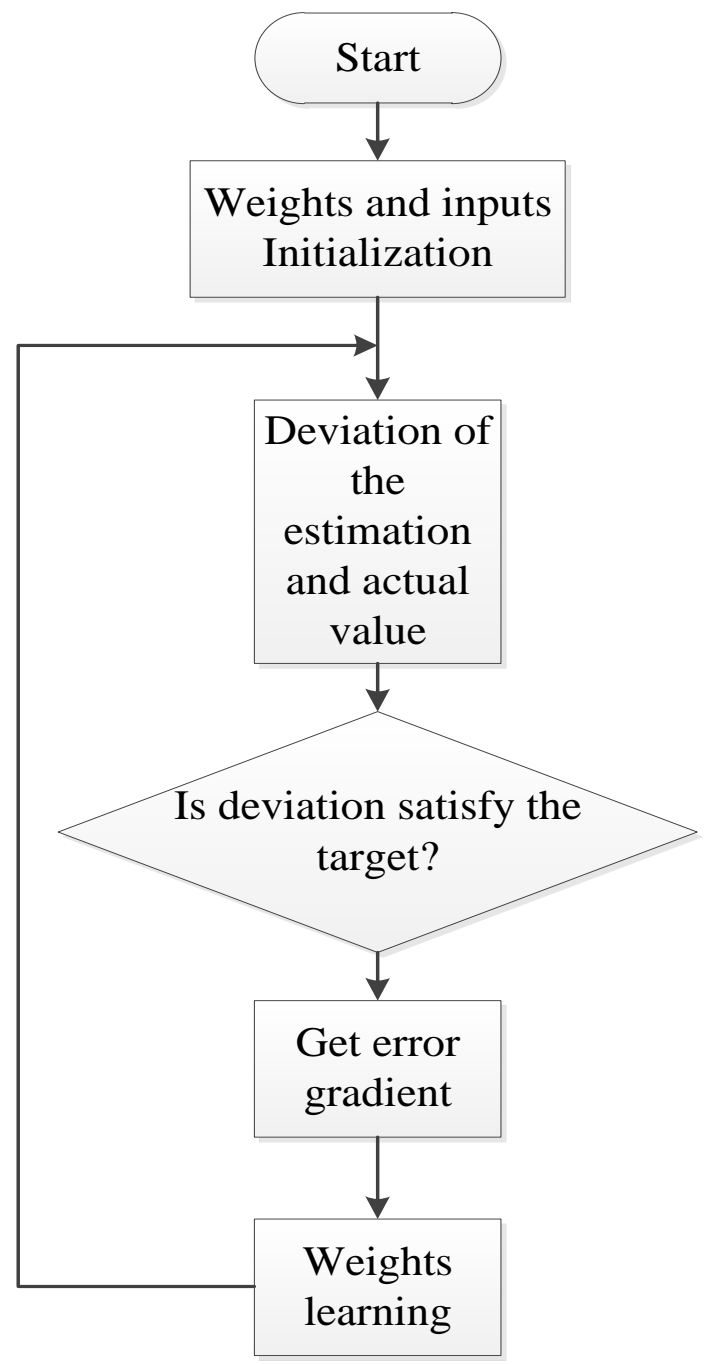

Figure 5-8 Flow chart of network algorithm

Figures 5-9 to 5-16 show the weights change during the network fitting the experimental data. The figures show the error between the actual voltage and the fitted voltage. These figures show that the parameter identification results are accurate. 


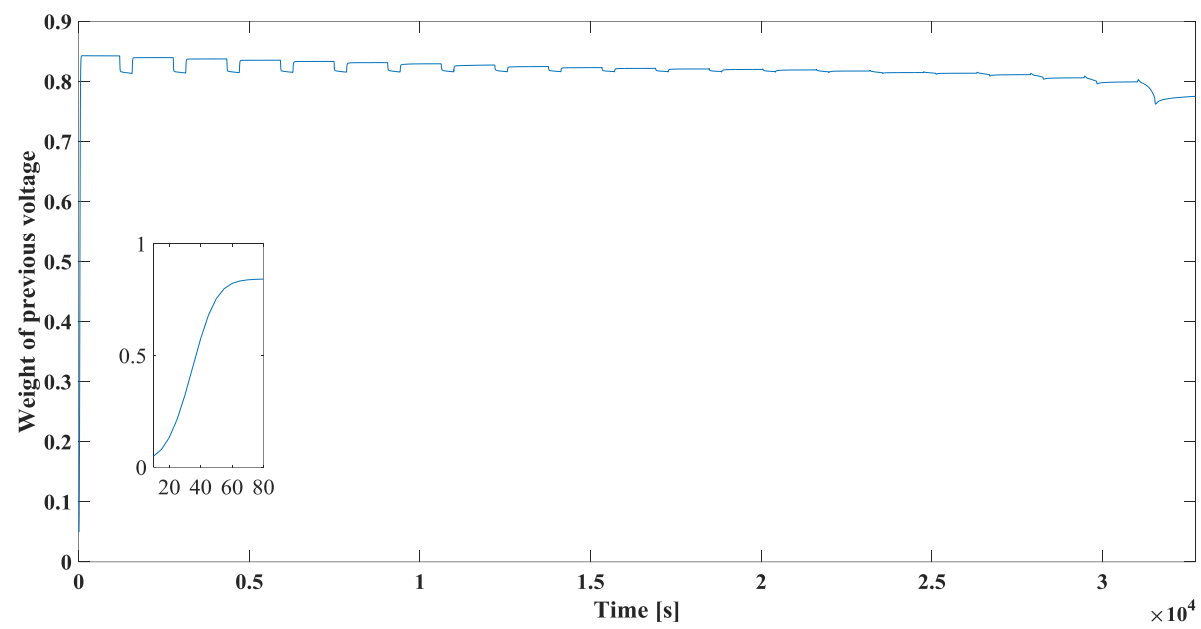

Figure 5-9 Weights of voltage

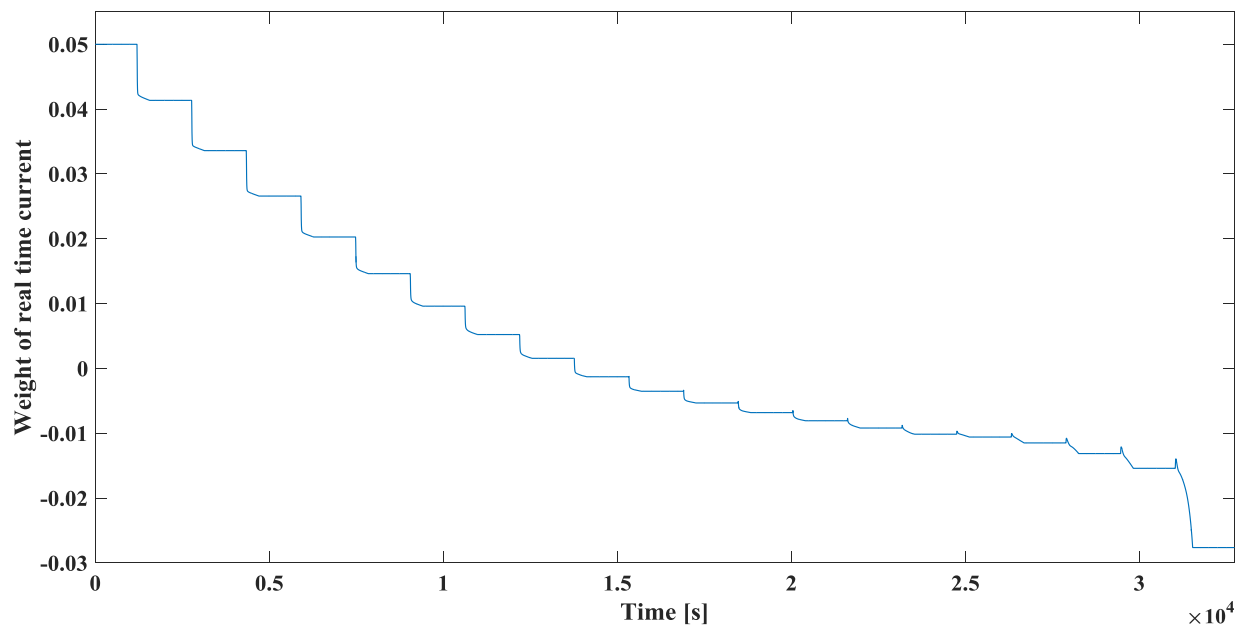

Figure 5-10 Weights of current

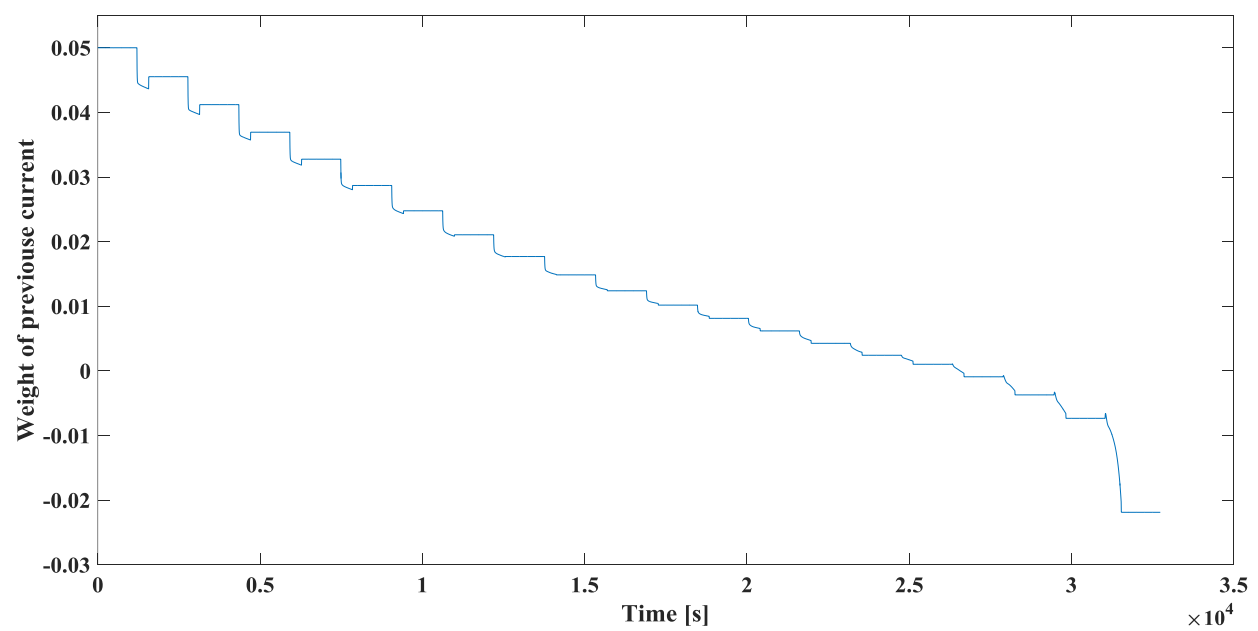

Figure 5-11 Weights of previous current 


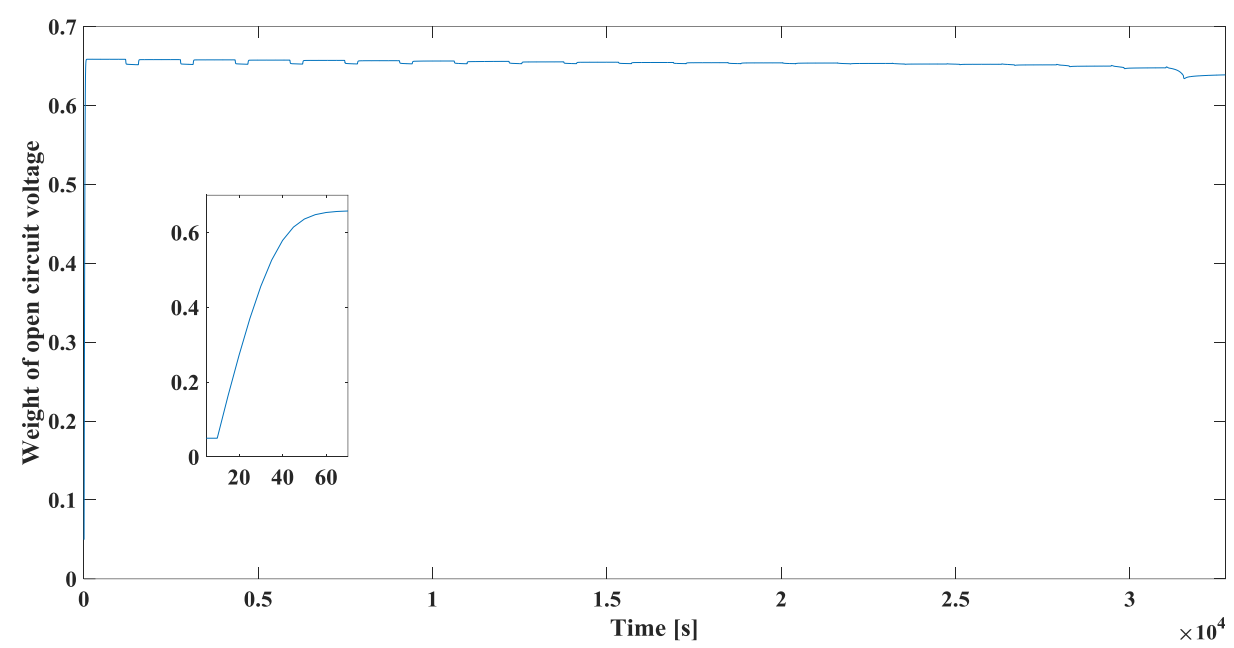

Figure 5-12 Weights of OCV

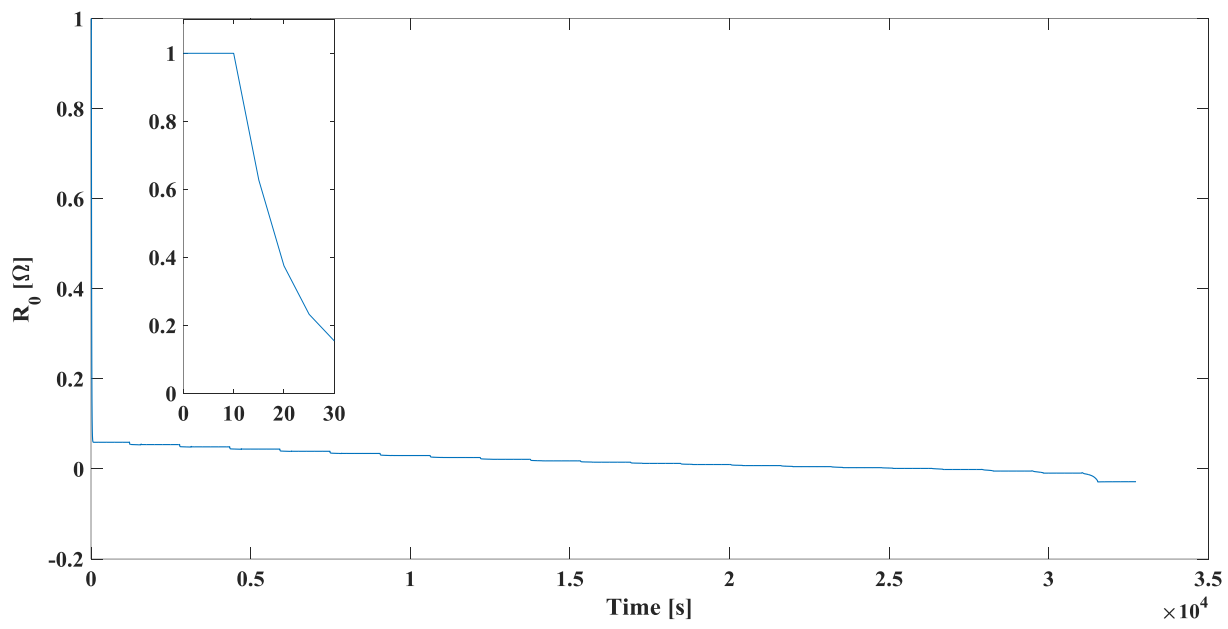

Figure 5-13 Weights of $R_{0}$

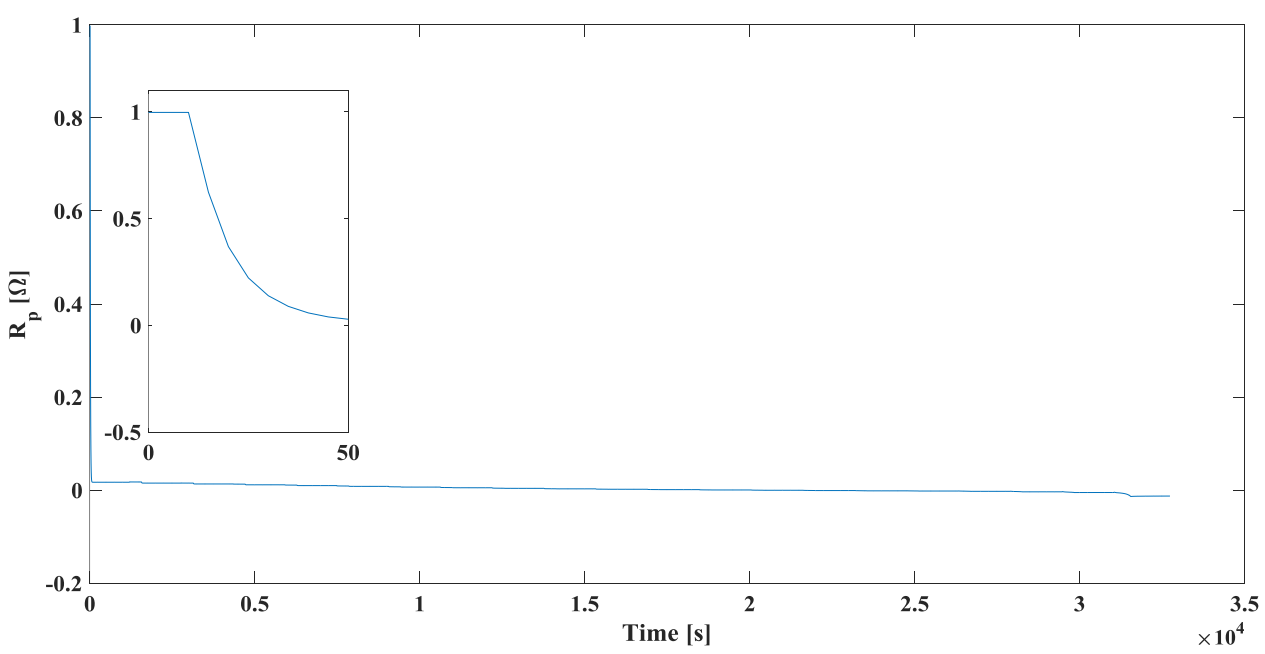

Figure 5-14 Weights of $R_{\mathrm{p}}$ 


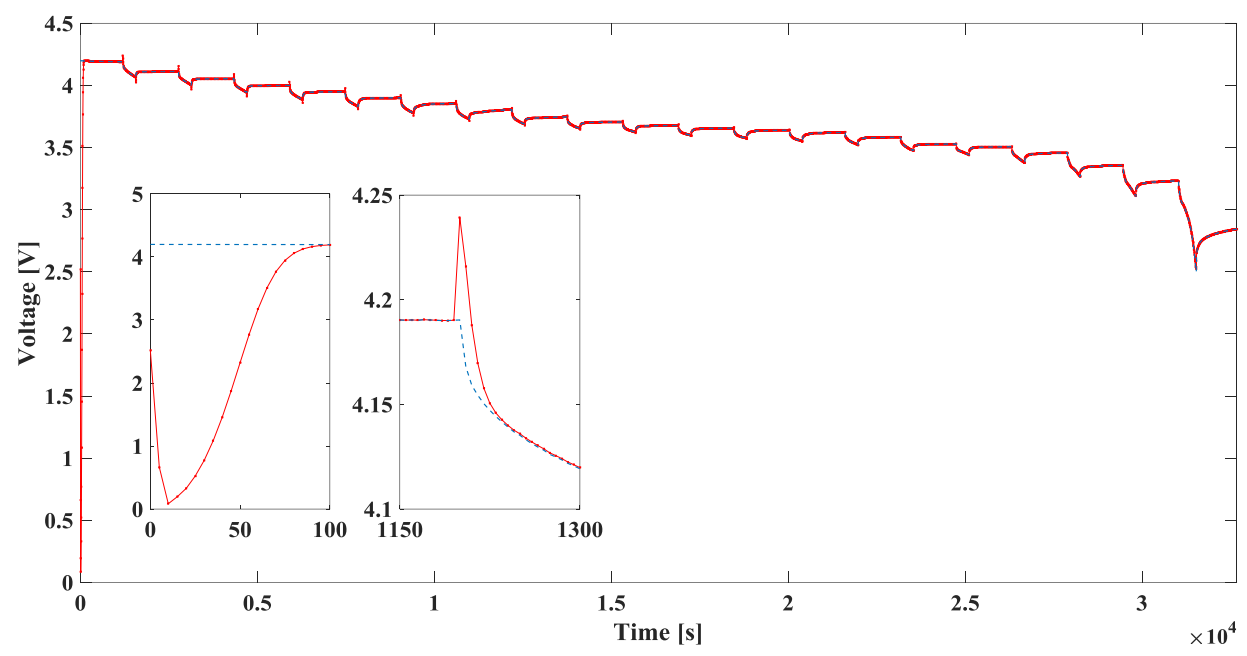

Figure 5-15 Fitted curve based on neural network

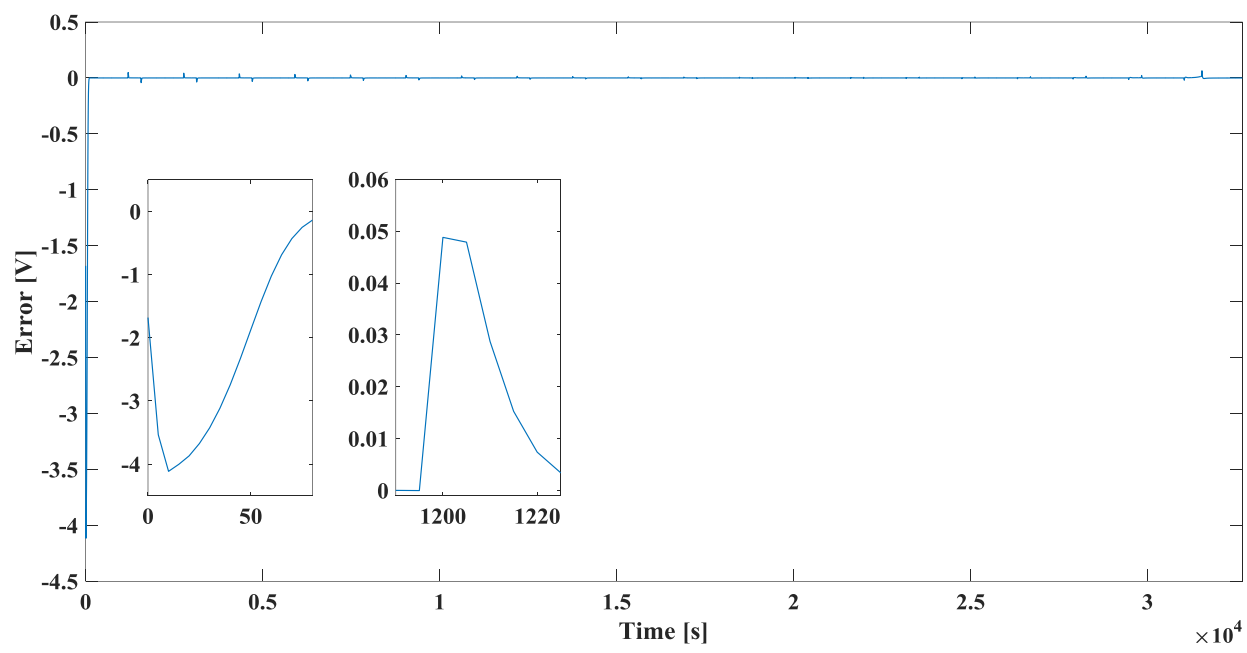

Figure 5-16 Error of fitting

\subsection{Simscape Verification}

Figures 5-17 and 5-18 show the Simscape simulation structure for both Thevenin model and improved Thevenin model. Considering the existing components in Simscape cannot satisfy the requirement to build the real battery, using Simcape language create the battery, resistance and capacitor as shown in figure 5-19. Figure 5-20 shows the signal builder that generate the input signal, the current is same as the discharge current used in the experiment. The code used to create this components are shown in the appendix. 


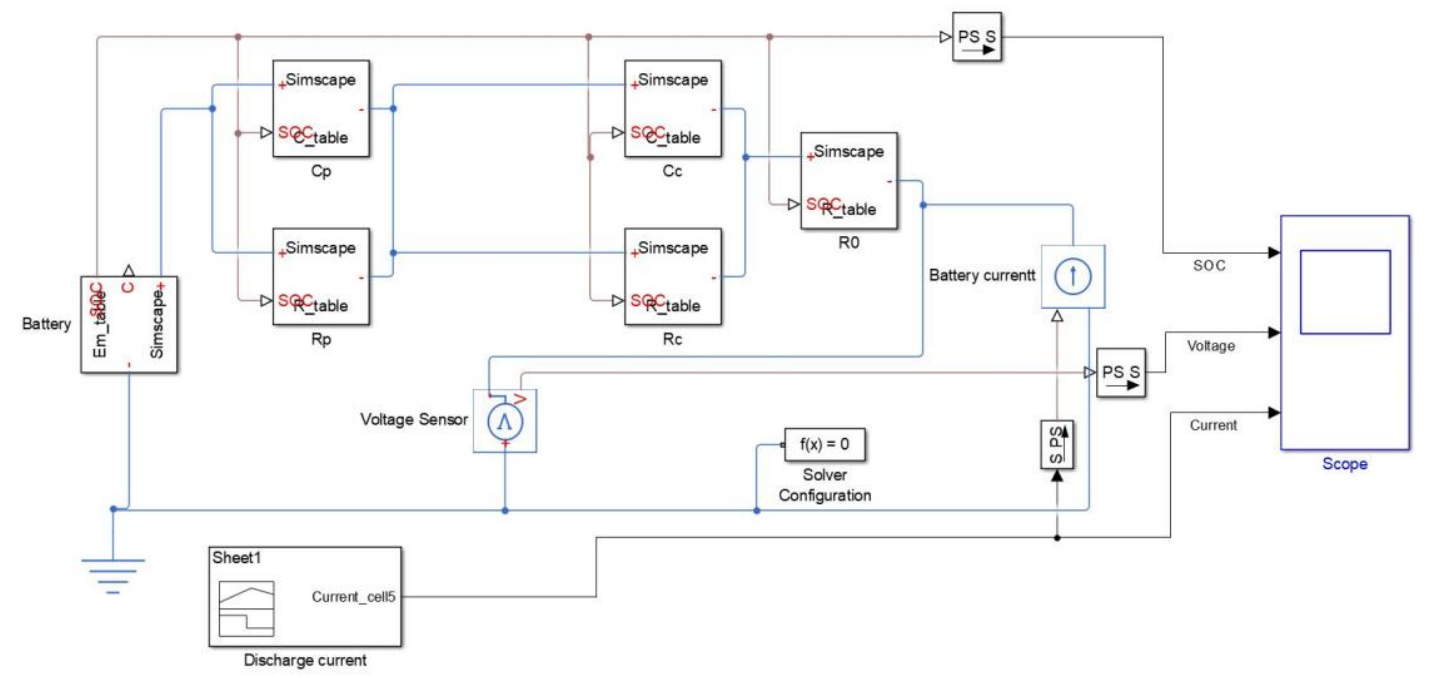

Figure 5-17 Simscape circuit of improved Thevenin model

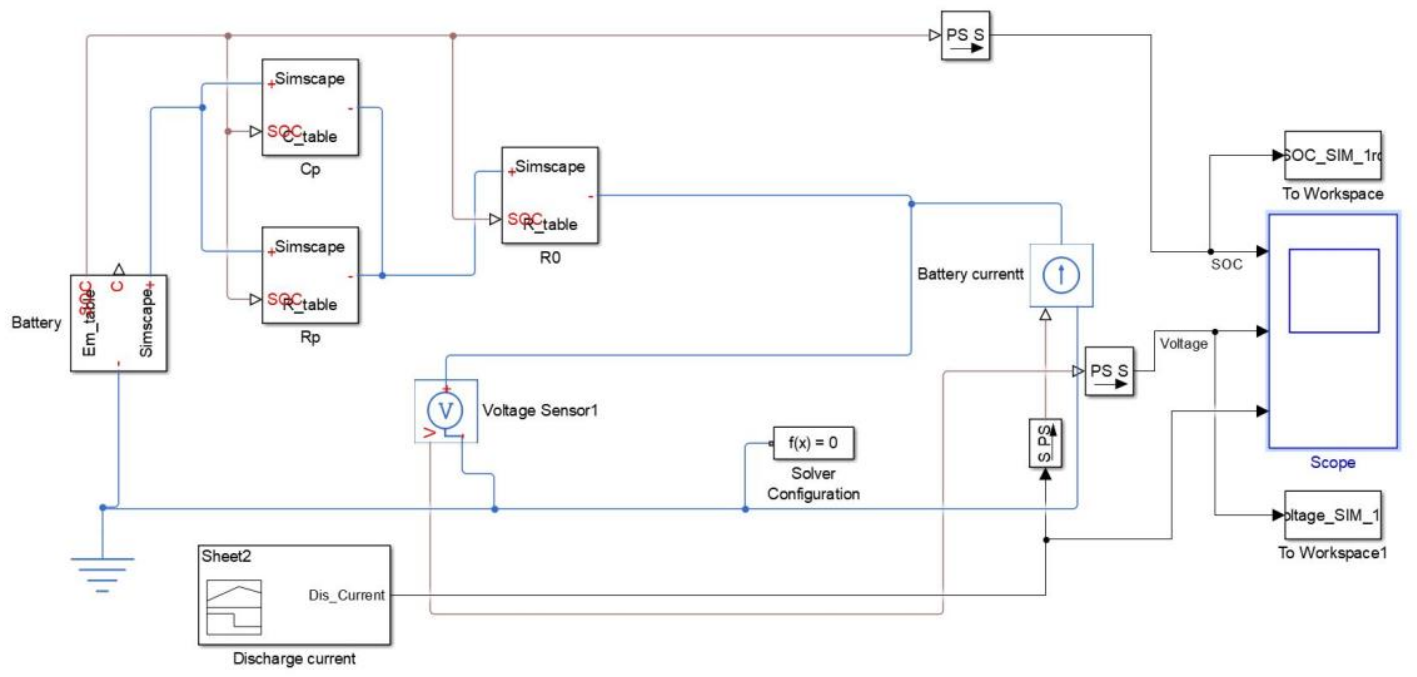

Figure 5-18 Simscape circuit of Thevenin model
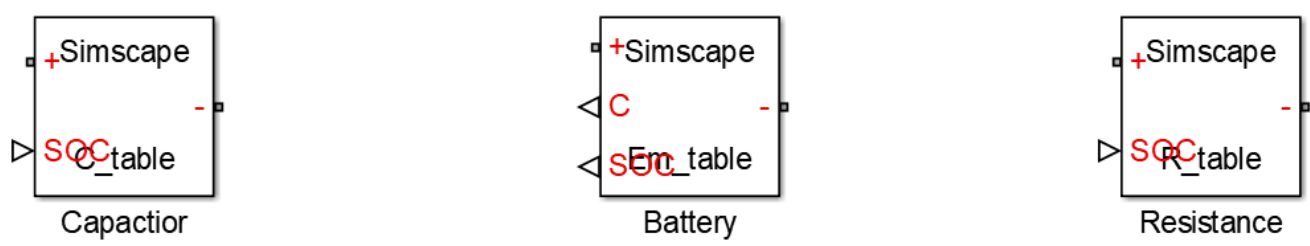

Figure 5-19 Created circuit components 


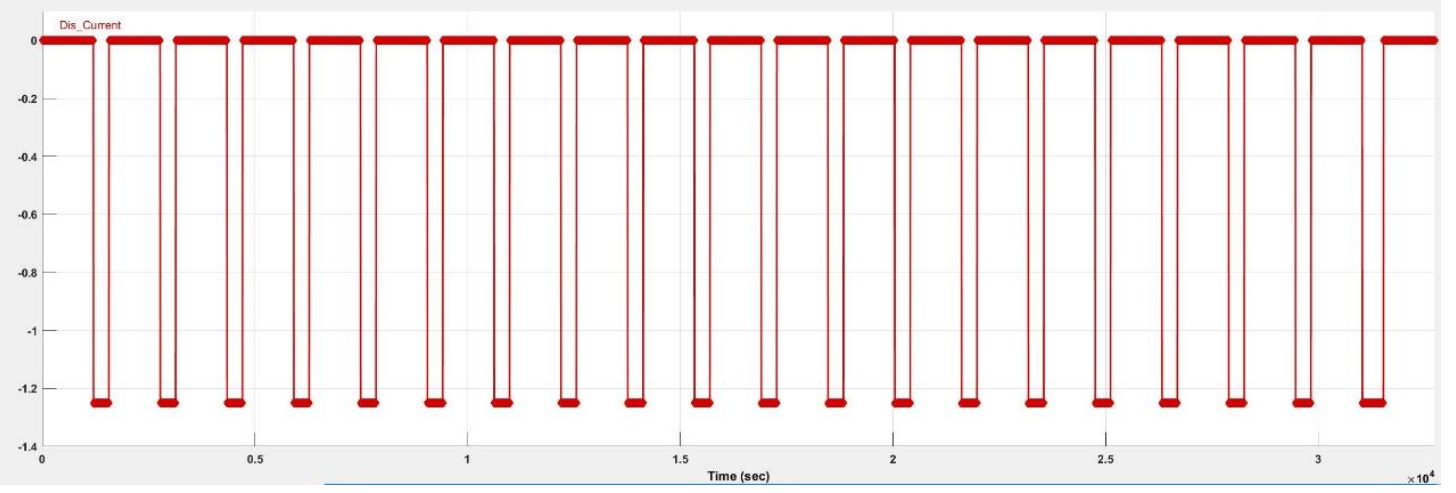

Figure 5-20 Signal builder of current

Figures 5-21 and 5-22 show the simulation results of Thevenin model and estimation error, it is observed that the model has the accurate estimation result at the most period, but the error is increased at the end of discharge due to polarization effect.

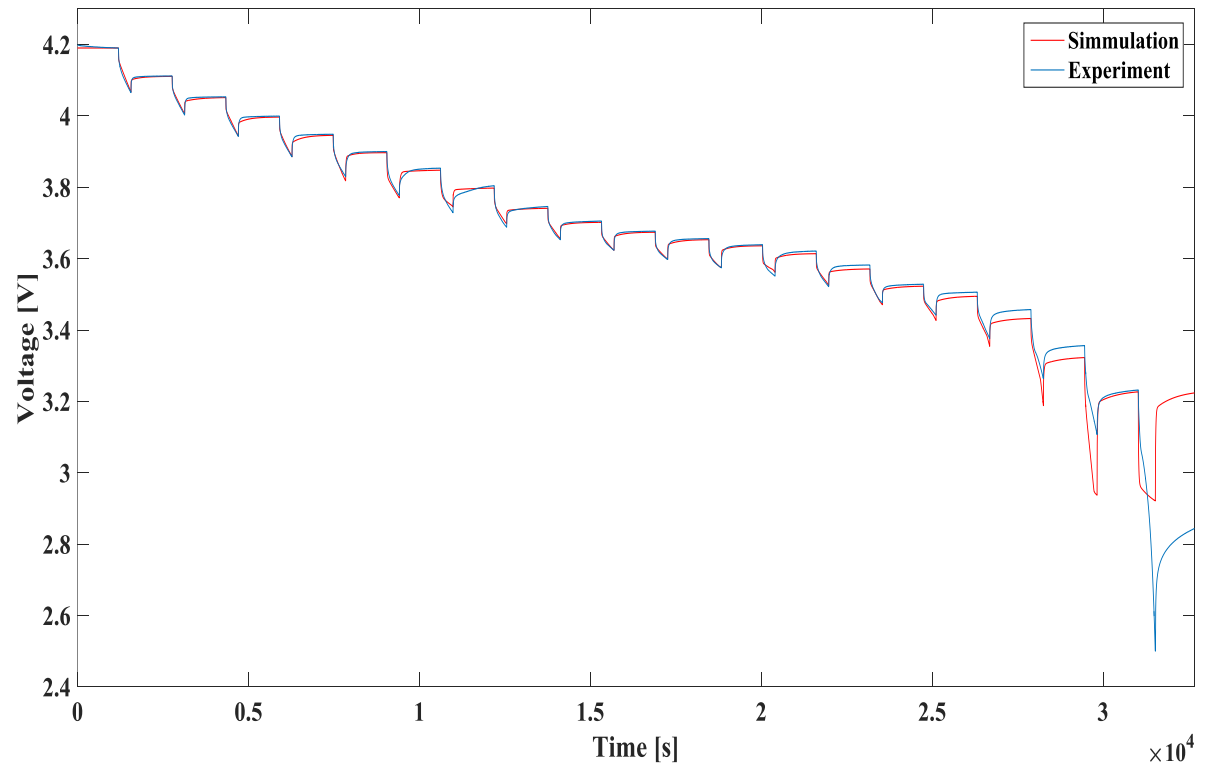

Figure 5-21 Simulation results of the Thevenin model and experiment data 


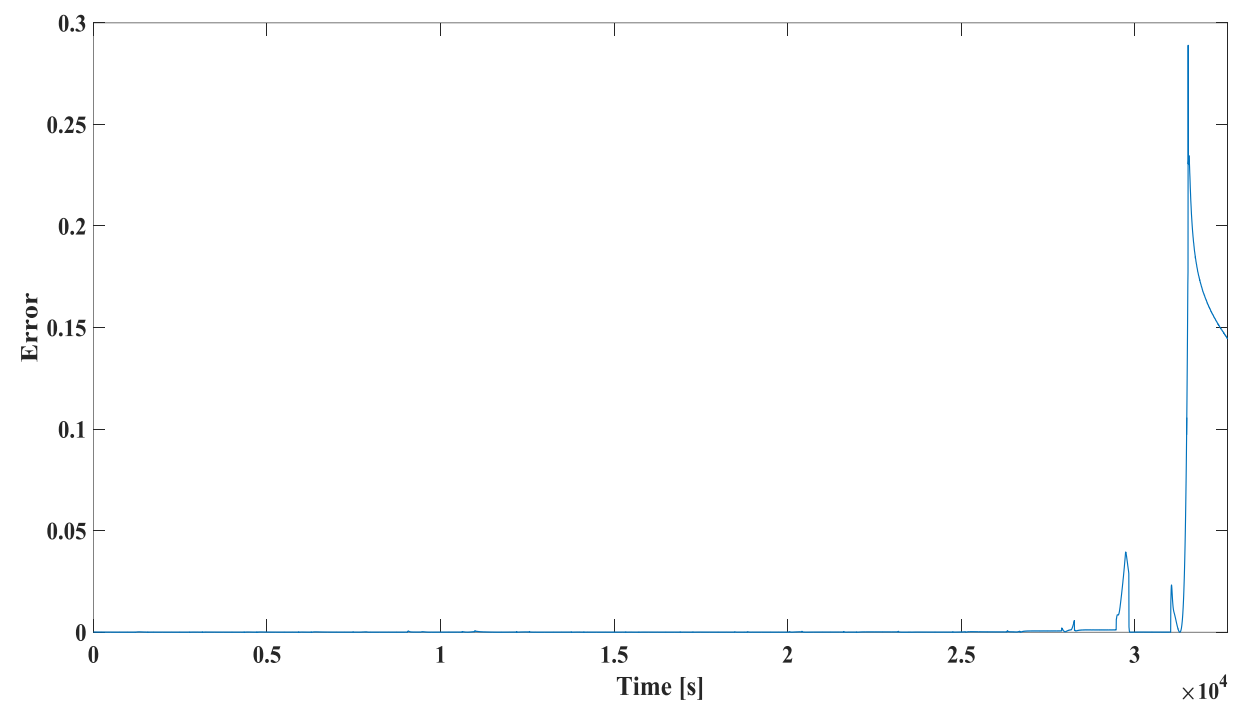

Figure 5-22 Estimation error of Thevenin model

Figures 5-23 and 5-24 show the simulation results of the improved Thevenin model and the estimation error. It is observed that the model has a better estimation result compare to the Thevenin model but still a deviation at some period exist.

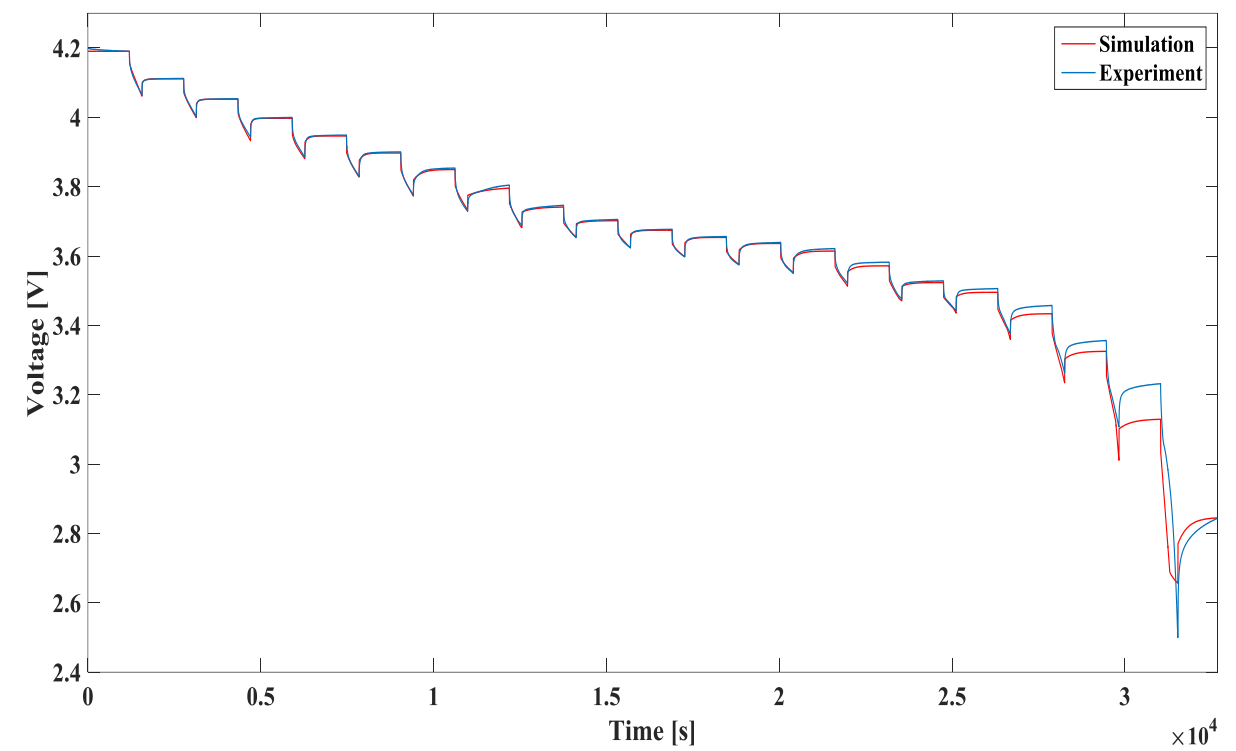

Figure 5-23 Simulation results of improved Thevenin model and experiment data 


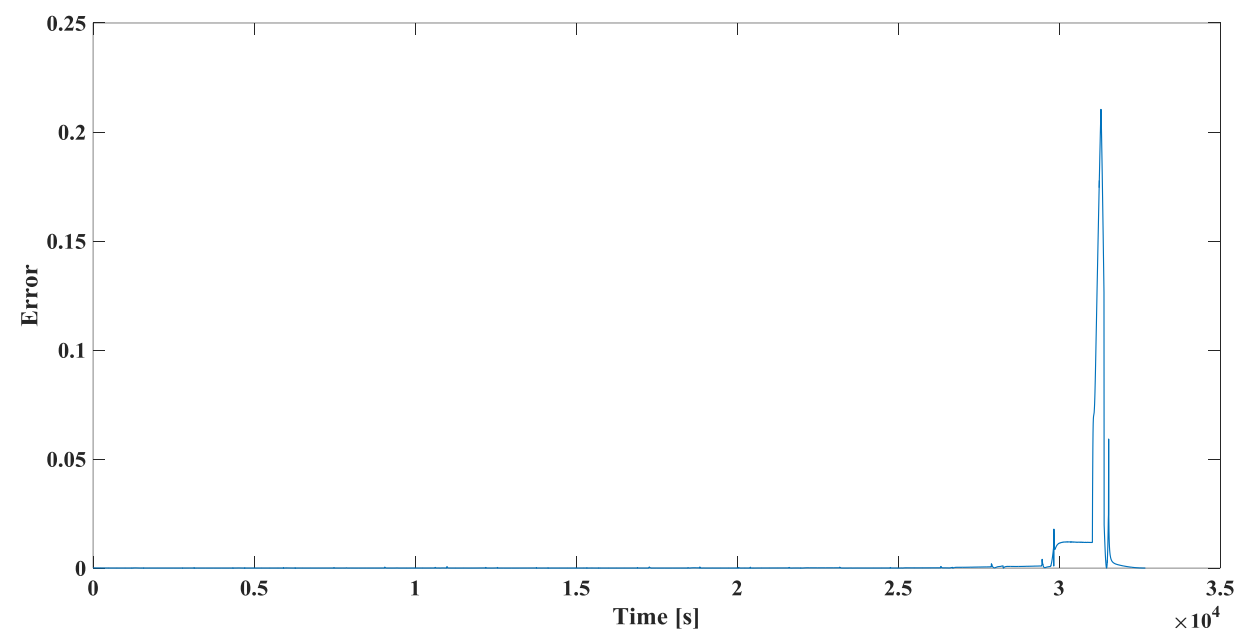

Figure 5-24 Estimation error of improved Thevenin model

Figures 5-25 and 5-26 show the simulation results of the variable order Thevenin model and the estimation error.

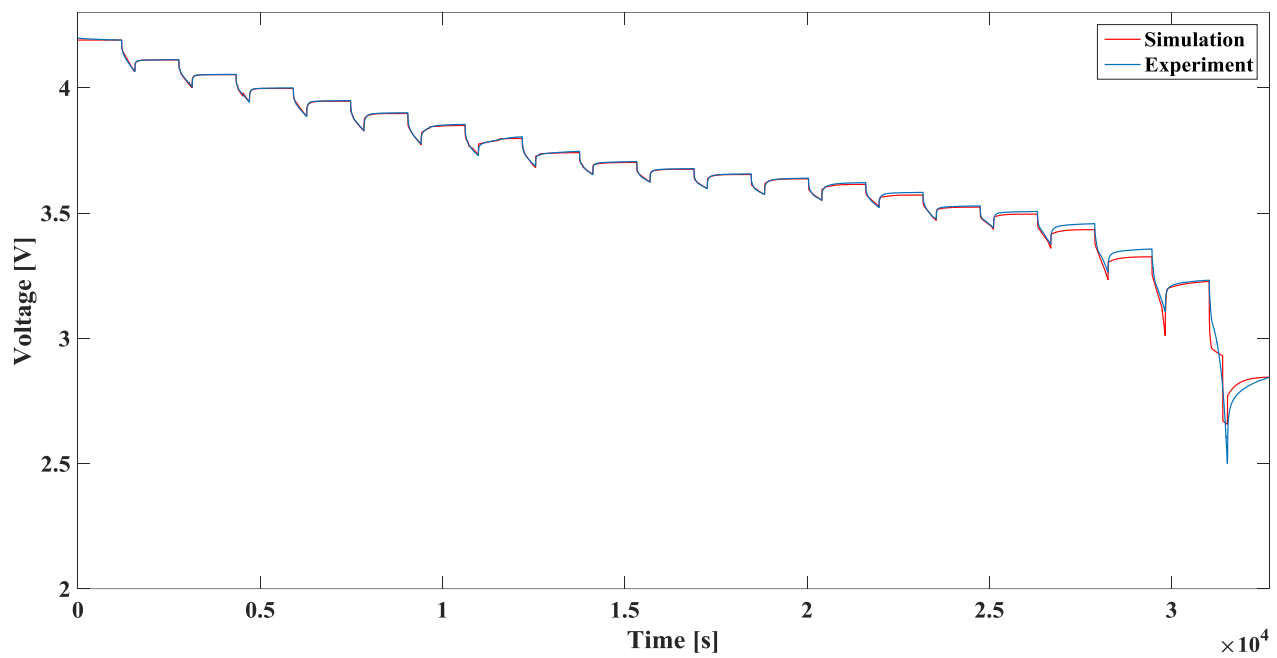

Figure 5-25 Simulation results of variable Thevenin model and experiment data 


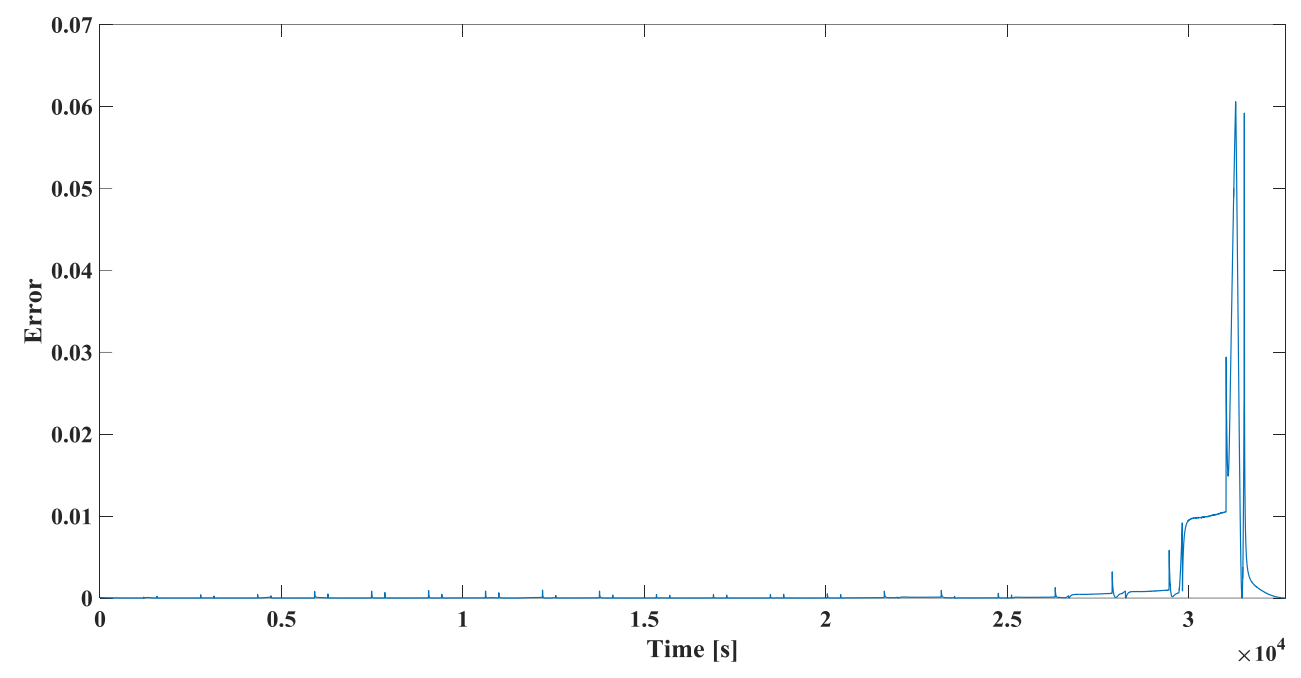

Figure 5-26 Estimation error of variable Thevenin model

The SOC estimation results and error are shown in figures 5-27 and 5-28.

Figure 5-28 compares the actual SOC with the estimation SOC by EKF and SOC estimation SOC by the proposed algorithm. Moreover, figure 5-28 shows comparison of SOC estimation error between the proposed algorithm and EKF. It demonstrated the satisfactory SOC estimation performance of the proposed algorithm.

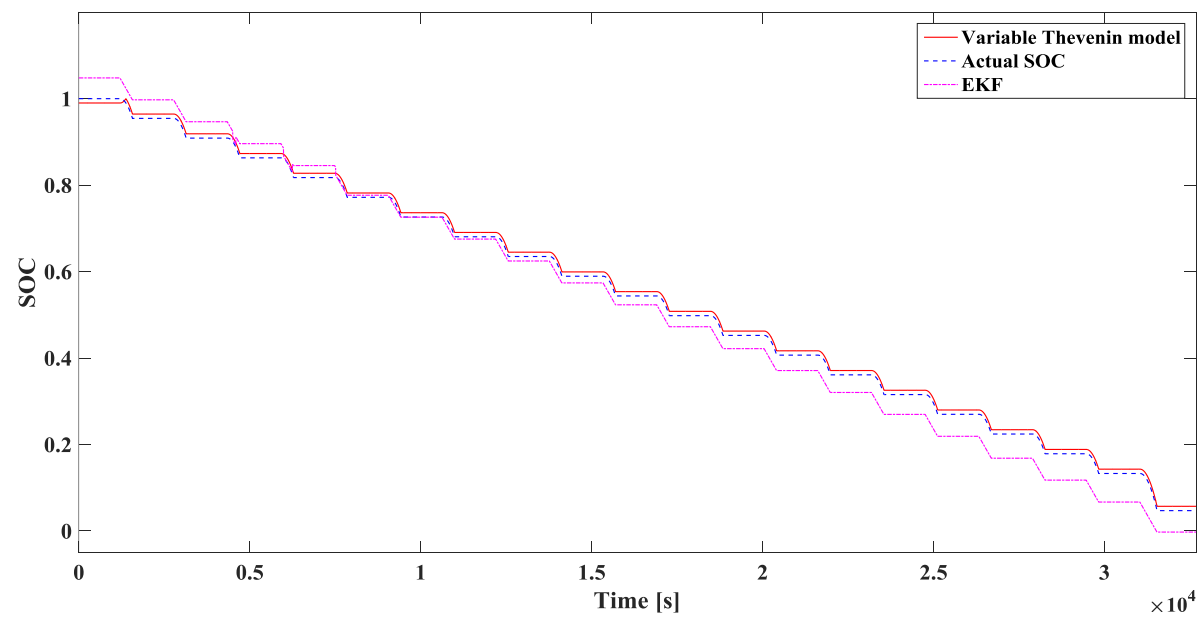

Figure 5-27 Comparison of the actual SOC, the SOC estimation by EKF, and the SOC estimation by variable Thevenin model 


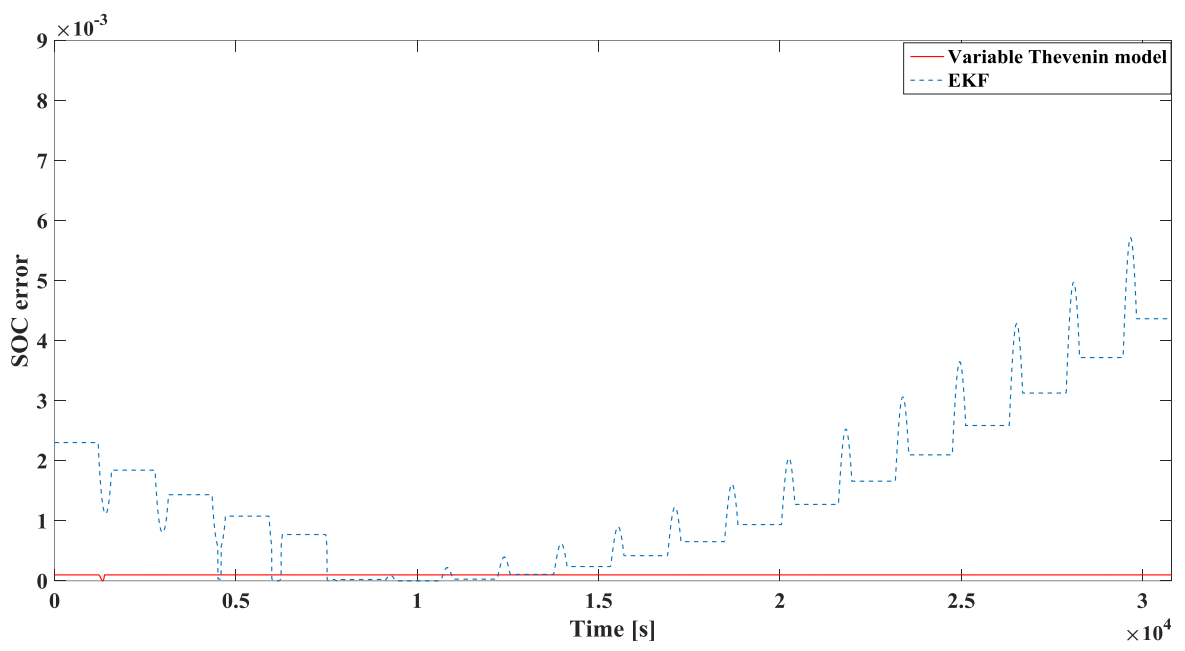

Figure 5-28 Comparison of SOC estimation error by proposed algorithm and EKF 


\section{CONCLUSION}

This thesis investigates a neural network based model for the SOC estimation in the Lithium-ion batteries. The neural network is used to identify the battery's parameters, then the identified parameters are provided to both Thevenin model and improved Thevenin model. The better estimation results were used to determine the SOC based on estimation error. The identification process is operating in four steps (1) battery's parameters identification based on the weights of the neural network. (2) setting the corresponding parameters for both models. (3) choosing the best battery model based on estimation error. (4) using the OCVSOC function to get an estimation of SOC.

In this paper, the open circuit voltage (OCV), the terminal cell voltage, and the impedance at certain frequencies are all used to estimate the SOC of the battery.

The K-means algorithm is used to process the experimental data to eliminate the measurement error and reduce the Sample differences. This study also used a linear neural network to identify the battery model parameters.

The Thevenin model has good estimation results in most discharge periods, however, at the end of discharge period, the error increases almost to $0.3 \mathrm{~V}$ due to the polarization effect. The improved Thevenin model consumes tremendous computation resources but has better estimation results. The proposed methods give the best SOC estimation result and combine the two advantages 1. have high

accuracy 2. low computational cost. Lastly, compare the proposed algorithm with classical SOC estimation algorithm. The results show that the proposed methods have much better estimation results than EKF. 


\section{REFERENCES}




\section{REFERENCES}

[1] H. Zhigang, C. Dong, P. Chaofeng, C. Long and W. Shaohua, "State of charge estimation of power Li-ion batteries using a hybrid estimation algorithm based on UKF", Electrochimica Acta, vol. 211, pp. 101-109, 2016.

[2] Z. Wei, B. Xiong, D. Ji and K. Tseng, "Online State of Charge and Capacity Dual Estimation with a Multi-timescale Estimator for Lithium-ion Battery", Energy Procedia, vol. 105, pp. 2953-2958, 2017.

[3] Z. Deng, L. Yang, Y. Cai and H. Deng, "Maximum Available Capacity and Energy Estimation Based on Support Vector Machine Regression for Lithium-ion Battery", Energy Procedia, vol. 107, pp. 68-75, 2017...

[4]. Y. Xing, W. He, M. Pecht and K. Tsui, "State of charge estimation of lithiumion batteries using the open-circuit voltage at various ambient temperatures", Applied Energy, vol. 113, pp. 106-115, 2014.

[5] Y. Chiang, W. Sean and J. Ke, "Online estimation of internal resistance and open-circuit voltage of lithium-ion batteries in electric vehicles", Journal of Power Sources, vol. 196, no. 8, pp. 3921-3932, 2011.

[6]. N. Yang, X. Zhang and G. Li, "State of charge estimation for pulse discharge of a LiFePO4 battery by a revised Ah counting", Electrochimica Acta, vol. 151, pp. 63-71, 2015.

[7]. R. Kuo, S. Hung and W. Cheng, "Application of an optimization artificial immune network and particle swarm optimization-based fuzzy neural network to an RFID-based positioning system", Information Sciences, vol. 262, pp. 78-98, 2014.

[8]. A. Hussein, "Capacity Fade Estimation in Electric Vehicle Li-Ion Batteries Using Artificial Neural Networks", IEEE Transactions on Industry Applications, vol. 51, no. 3, pp. 2321-2330, 2015.

[9]. X. Dang, L. Yan, K. Xu, X. Wu, H. Jiang and H. Sun, "Open-Circuit VoltageBased State of Charge Estimation of Lithium-ion Battery Using Dual Neural Network Fusion Battery Model", Electrochimica Acta, vol. 188, pp. 356-366, 2016.

[10]. H. Shi, Z. Yu and L. Zhu, "Optimal estimation for vibration responses of vehicle and track based on UKF", JOURNAL OF ELECTRONIC MEASUREMENT AND INSTRUMENT, vol. 26, no. 4, pp. 279-285, 2013. 
[11]. B. Xia, H. Wang, Y. Tian, M. Wang, W. Sun and Z. Xu, "State of Charge Estimation of Lithium-Ion Batteries Using an Adaptive Cubature Kalman Filter", Energies, vol. 8, no. 6, pp. 5916-5936, $2015 .$.

[12]. Hongwen He, Rui Xiong, Xiaowei Zhang, Fengchun Sun and JinXin Fan, "State-of-Charge Estimation of the Lithium-Ion Battery Using an Adaptive Extended Kalman Filter Based on an Improved Thevenin Model", IEEE Transactions on Vehicular Technology, vol. 60, no. 4, pp. 1461-1469, 2011.

[13]. B. Pattipati, C. Sankavaram and K. Pattipati, "System Identification and Estimation Framework for Pivotal Automotive Battery Management System Characteristics", IEEE Transactions on Systems, Man, and Cybernetics, Part C (Applications and Reviews), vol. 41, no. 6, pp. 869-884, 2011.

[14]. S. Nejad, D. Gladwin and D. Stone, "A systematic review of lumpedparameter equivalent circuit models for real-time estimation of lithium-ion battery states", Journal of Power Sources, vol. 316, pp. 183-196, 2016.

[15]. L. Yao, W. Prayun, M. Abdul Aziz and T. Sutikno, "Battery State-of-Charge Estimation with Extended Kalman-Filter using Third-Order Thevenin Model", TELKOMNIKA (Telecommunication Computing Electronics and Control), vol. 13, no. 2, p. 401, 2015.

[16]. A. Vasebi, M. Partovibakhsh and S. Bathaee, "A novel combined battery model for state-of-charge estimation in lead-acid batteries based on extended Kalman filter for hybrid electric vehicle applications", Journal of Power Sources, vol. 174, no. 1, pp. 30-40, 2007.

[17]. M. Wang, A. Le, D. Noelle, Y. Shi, Y. Meng and Y. Qiao, "Internal-shortmitigating current collector for lithium-ion battery", Journal of Power Sources, vol. 349, pp. 84-93, 2017.

[18]. R. Masoudi, T. Uchida and J. McPhee, "Parameter estimation of an electrochemistry-based lithium-ion battery model", Journal of Power Sources, vol. 291, pp. 215-224, 2015.

[19]. H. He, X. Zhang, R. Xiong, Y. Xu and H. Guo, "Online model-based estimation of state-of-charge and open-circuit voltage of lithium-ion batteries in electric vehicles", Energy, vol. 39, no. 1, pp. 310-318, 2012.

[20]. D. Li, J. Ouyang, H. Li and J. Wan, "State of charge estimation for LiMn2O4 power battery based on strong tracking sigma point Kalman filter", Journal of Power Sources, vol. 279, pp. 439-449, 2015. 
[21]. B. Xia, H. Wang, M. Wang, W. Sun, Z. Xu and Y. Lai, "A New Method for State of Charge Estimation of Lithium-Ion Battery Based on Strong Tracking Cubature Kalman Filter", Energies, vol. 8, no. 12, pp. 13458-13472, 2015.

[22]. B. Xia, Z. Sun, R. Zhang and Z. Lao, "A Cubature Particle Filter Algorithm to Estimate the State of the Charge of Lithium-Ion Batteries Based on a Second-Order Equivalent Circuit Model", Energies, vol. 10, no. 4, p. 457, 2017.

[23] M. Qi and G. Zhang, "An investigation of model selection criteria for neural network time series forecasting", European Journal of Operational Research, vol. 132, no. 3, pp. 666-680, 2001.

[24] K. Burnham, D. Anderson and K. Huyvaert, "AIC model selection and multimodel inference in behavioral ecology: some background, observations, and comparisons", Behavioral Ecology and Sociobiology, vol. 65, no. 1, pp. 23-35, 2010.

[25] D. Kim, T. Goh, M. Park and S. Kim, "Fuzzy Sliding Mode Observer with Grey Prediction for the Estimation of the State-of-Charge of a Lithium-Ion Battery", Energies, vol. 8, no. 11, pp. 12409-12428, 2015.

[26] J. Klee Barillas, J. Li, C. Günther and M. Danzer, "A comparative study and validation of state estimation algorithms for Li-ion batteries in battery management systems", Applied Energy, vol. 155, pp. 455-462, 2015.

[27] H. Chaoui, C. Ibe-Ekeocha and H. Gualous, "Aging prediction and state of charge estimation of a LiFePO 4 battery using input time-delayed neural networks", Electric Power Systems Research, vol. 146, pp. 189-197, 2017.

[28] Y. Tian, D. Li, J. Tian and B. Xia, "State of charge estimation of lithium-ion batteries using an optimal adaptive gain nonlinear observer", Electrochimica Acta, vol. 225, pp. 225-234, 2017.

[29] P. Kumudha and R. Venkatesan, "Cost-Sensitive Radial Basis Function Neural Network Classifier for Software Defect Prediction", The Scientific World Journal, vol. 2016, pp. 1-20, 2016.

[30] A. Nikolian, Y. Firouz, R. Gopalakrishnan, J. Timmermans, N. Omar, P. van den Bossche and J. van Mierlo, "Lithium Ion Batteries-Development of Advanced Electrical Equivalent Circuit Models for Nickel Manganese Cobalt Lithium-Ion", Energies, vol. 9, no. 5, p. 360, 2016. 
APPENDIX: MATLAB CODE 


\section{$\underline{\text { Functions }}$}

The following function is main coding where developed using Matlab to create the Scimscape blocks, and to find the battery parameters for building Thevenin model and improved model.

\section{Create new components for battery model}

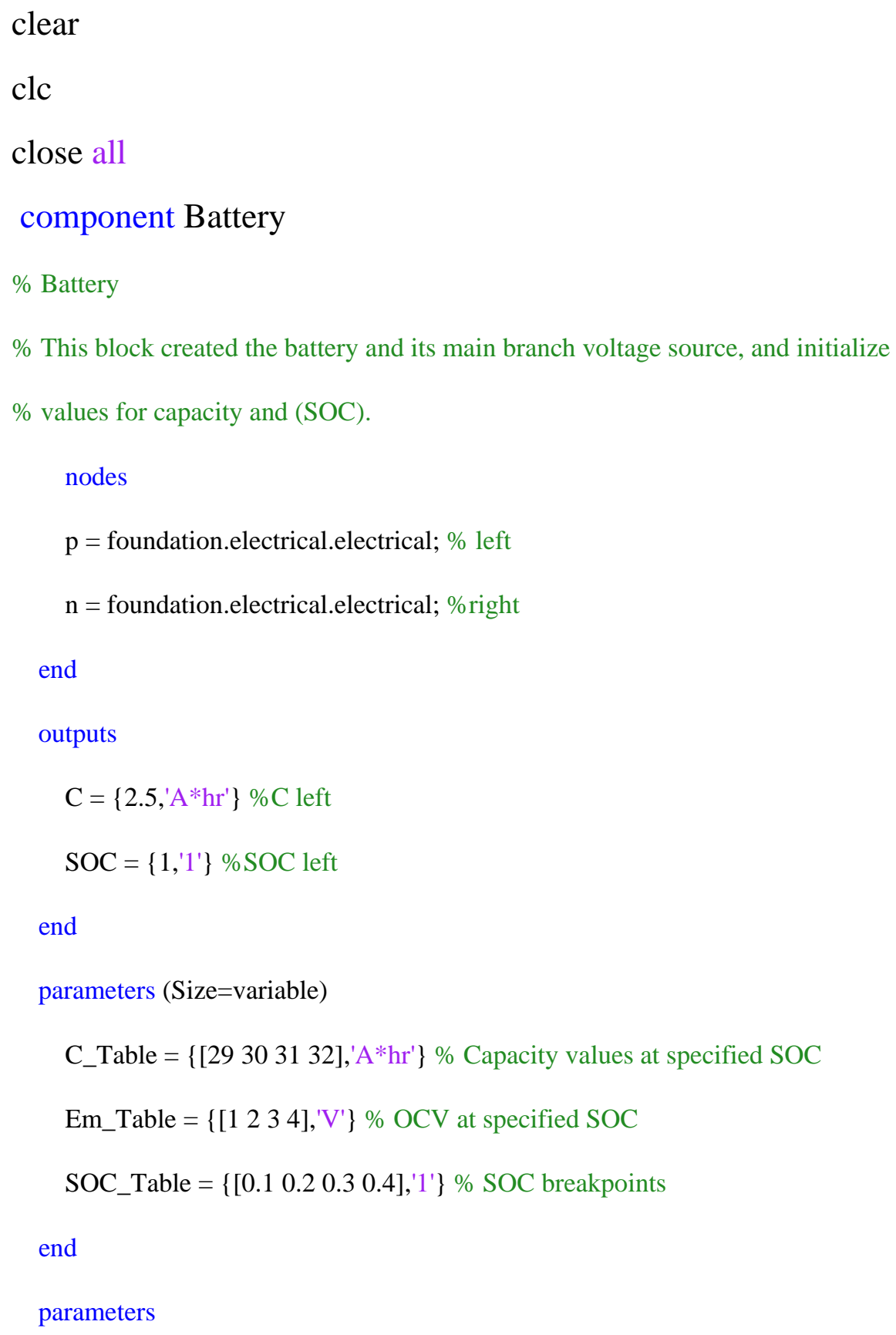




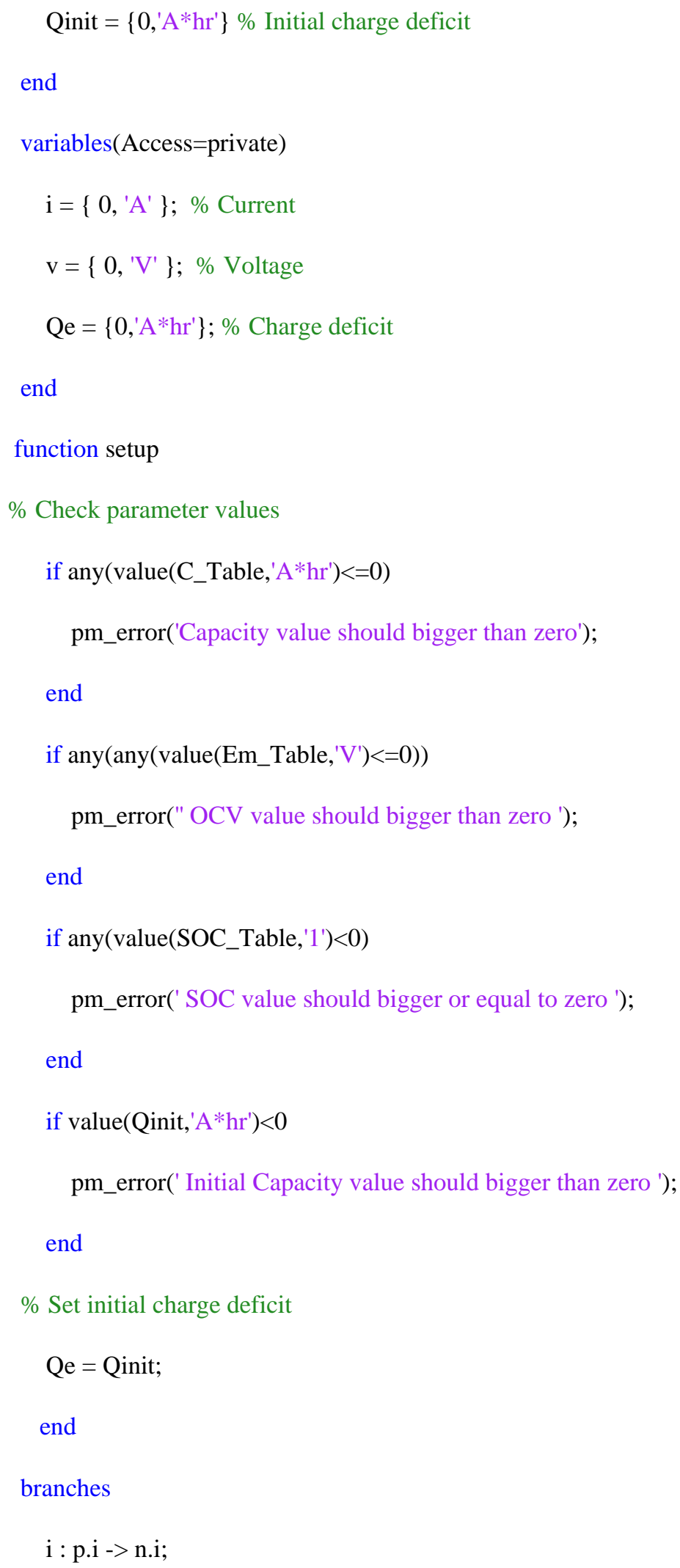




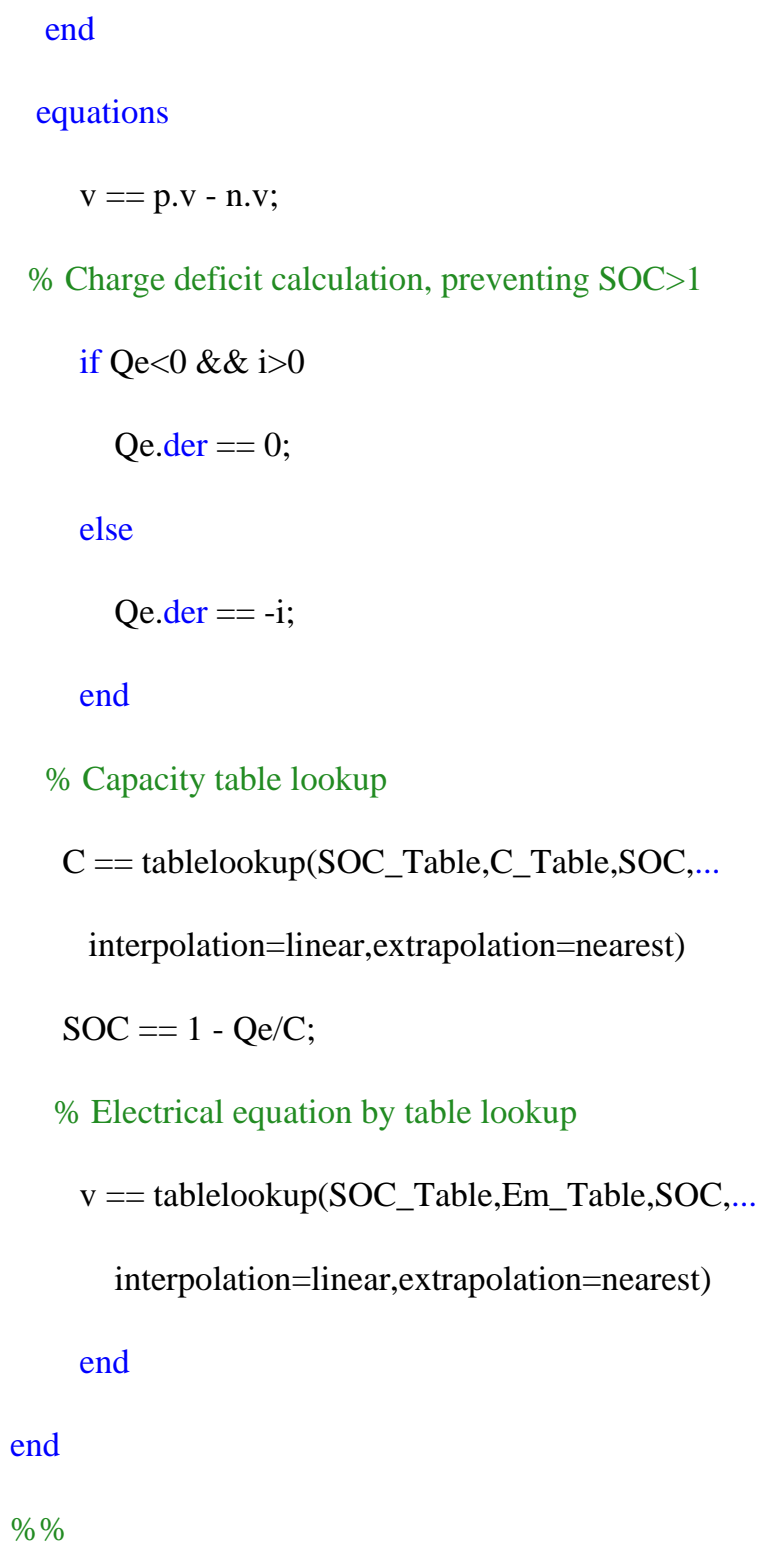


$\mathrm{n}=$ foundation.electrical.electrical;

end

inputs

$$
\operatorname{SOC}=\left\{1,1^{\prime}\right\}
$$

end

parameters $($ Size $=$ variable $)$

C_Table $=\left\{\left[\begin{array}{llll}0.1 & 0.2 & 0.3 & 0.4\end{array}\right],,^{\prime}{ }^{\prime}\right\}$

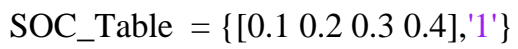

end

parameters

$\mathrm{v} 0=\left\{0,{ }^{\prime} \mathrm{V}^{\prime}\right\} ; \%$ Initial voltage across capacitor

end

variables(Access $=$ private $)$

$\mathrm{i}=\left\{0, \mathrm{~A}^{\prime}\right\}$;

$\mathrm{v}=\left\{0, \mathrm{C}^{\prime}\right\}$;

end

function setup

$\%$ Check parameter values

if any (any (value $\left(\right.$ C_Table, 'F' $\left.{ }^{\prime}<=0\right)$ )

pm_error(' Capacity value should bigger than zero ')

end

if any $($ value $($ SOC_Table, '1' 1 < $<0)$

pm_error(' SOC value should bigger or equal to zero ');

end

$\%$ Set the initial voltage

$\mathrm{v}=\mathrm{v} 0$ 


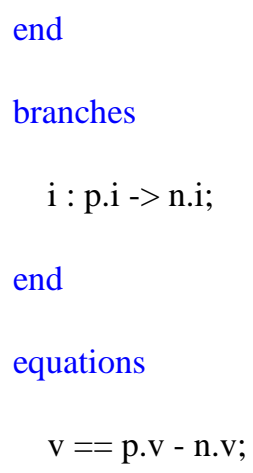

component Resistance

$\%$ Resistance

$\%$ Models a resistor where the resistance value depends on an SOC

nodes

$$
\begin{aligned}
& \qquad \mathrm{p}=\text { foundation.electrical.electrical; } \\
& \mathrm{n}=\text { foundation.electrical.electrical; }
\end{aligned}
$$




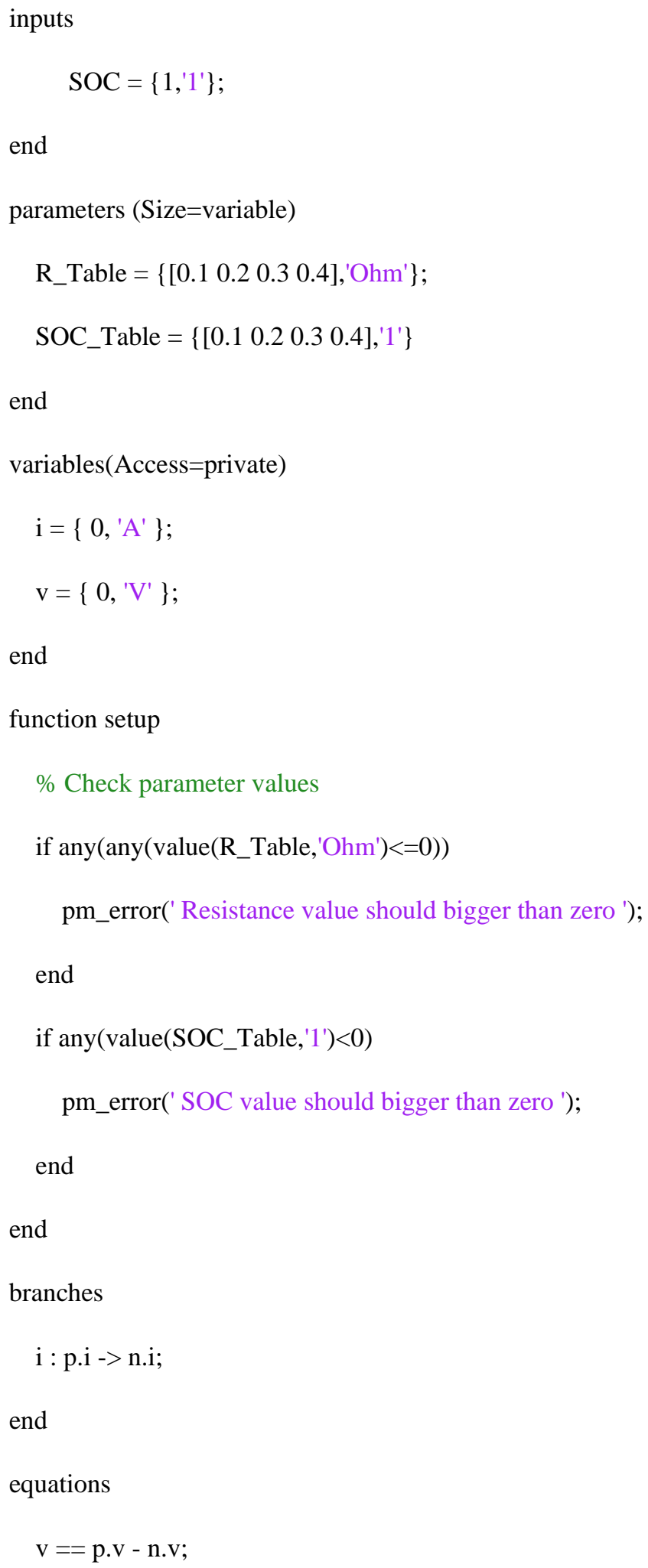




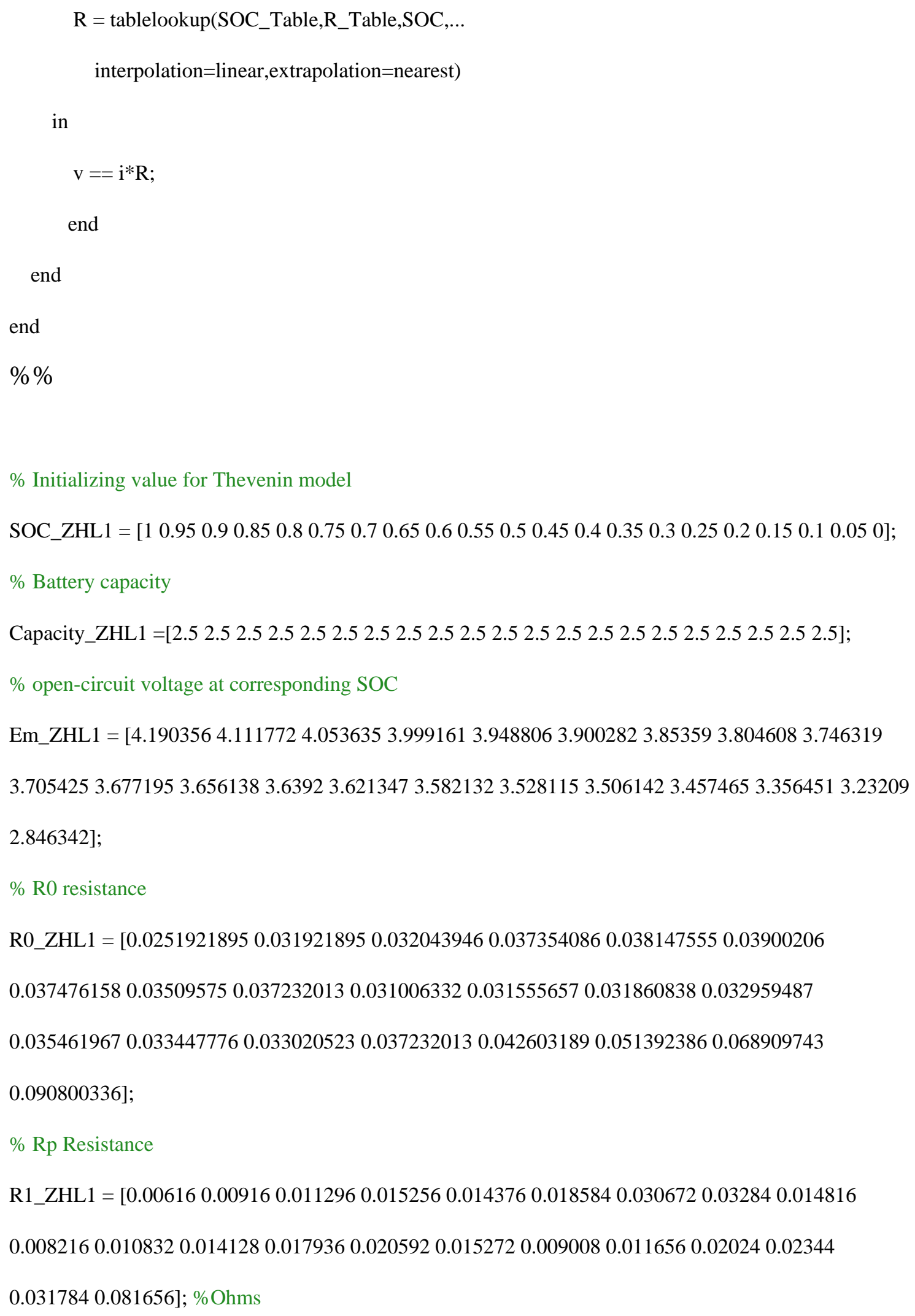




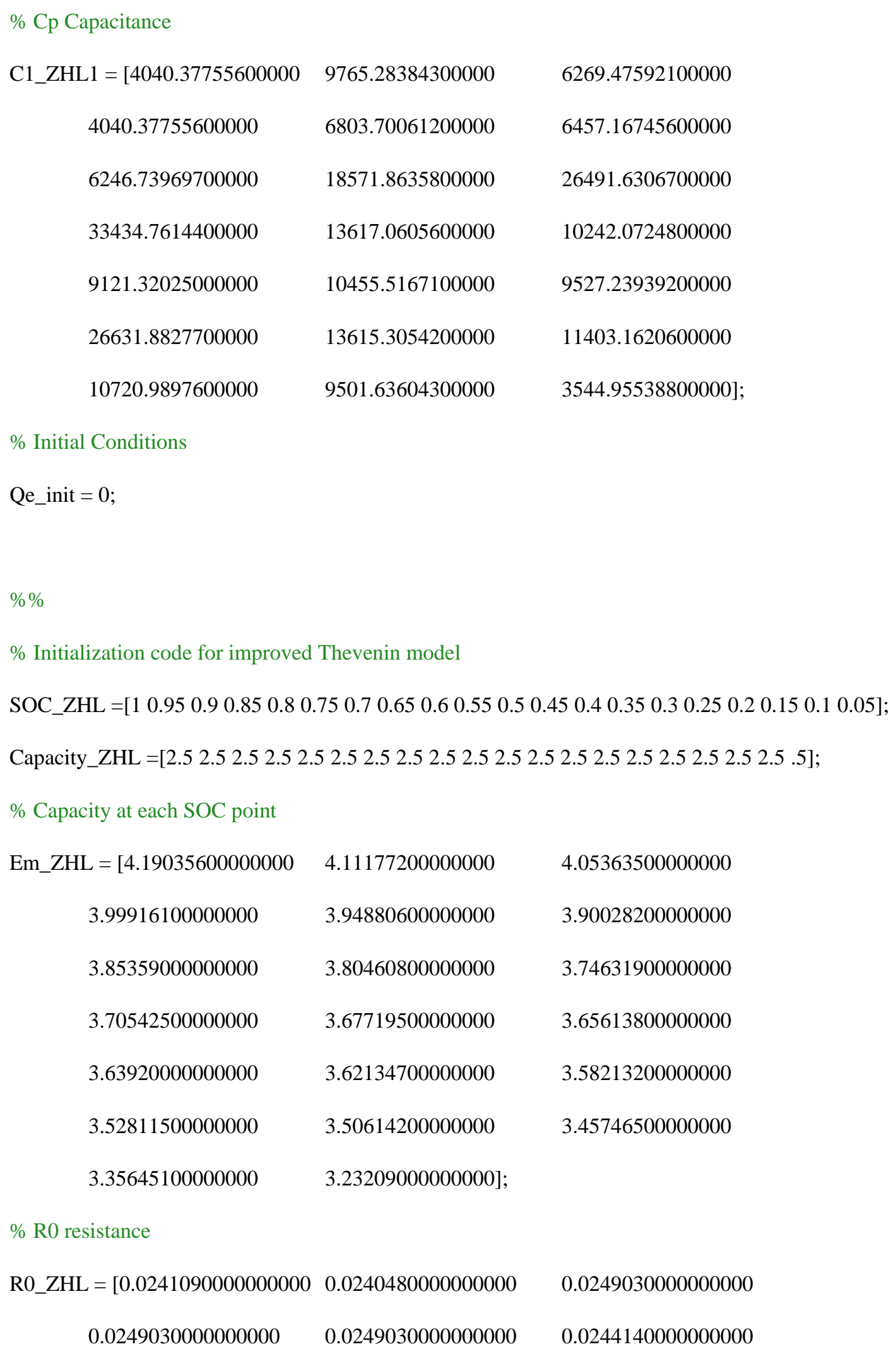




\begin{tabular}{|c|c|c|}
\hline 0.0245980000000000 & 0.0245980000000000 & 0.0239870000000000 \\
\hline 0.0234380000000000 & 0.0235600000000000 & 0.0236820020000000 \\
\hline 0.0239260000000000 & 0.0239260000000000 & 0.0242920000000000 \\
\hline 0.0254520000000000 & 0.0274050000000000 & 0.0302130000000000 \\
\hline 0.0361330000000000 & $0.0678720000000000]^{\prime}$ & \\
\hline \multicolumn{3}{|l|}{$\%$ Rp Resistance } \\
\hline \multicolumn{2}{|c|}{$\mathrm{R} 1 \_\mathrm{ZHL}=[0.003903200000000000 .00375280000000000$} & 0.00379920000000000 \\
\hline 0.00519600000000000 & 0.00656640000000000 & 0.0295040000000000 \\
\hline 0.0347440000000000 & 0.0143120000000000 & 0.00639280000000000 \\
\hline 0.00584400000000000 & 0.00732480000000000 & 0.00898400000000000 \\
\hline 0.0152720000000000 & 0.00736000000000000 & 0.00661360000000000 \\
\hline 0.00612720000000000 & 0.0146000000000000 & 0.0176160000000000 \\
\hline 0.0264160000000000 & $0.109280000000000]$ & \\
\hline \multicolumn{3}{|l|}{$\% \mathrm{Cp}$} \\
\hline C1_ZHL $=[2808.72618100000$ & 4961.81242100000 & 3569.94380300000 \\
\hline 1848.09304200000 & 987.779658800000 & 335.749071100000 \\
\hline 821.361433700000 & 1524.30267300000 & 2218.59068600000 \\
\hline 1422.74945900000 & 979.876666200000 & 689.176775100000 \\
\hline 407.429883000000 & 807.705168400000 & 1463.22494000000 \\
\hline 1953.66715400000 & 691.872236400000 & 655.140267800000 \\
\hline 473.516219300000 & 130.971819300000]; & \\
\hline \multicolumn{3}{|c|}{ \% Rc Resistance Rc_ZHL = [0.00950400000000000 0.0120000000000000} \\
\hline 0.0237440000000000 & 0.0240560000000000 & 0.00798880000000000 \\
\hline 0.0108880000000000 & 0.0108880000000000 & 0.0114480000000000 \\
\hline 0.0125680000000000 & 0.0146960000000000 & 0.0176560000000000 \\
\hline 0.0148240000000000 & 0.0158720000000000 & 0.00981600000000000 \\
\hline
\end{tabular}




$$
\begin{aligned}
& \begin{array}{lll}
0.0178320000000000 & 0.0211360000000000 & 0.0234640000000000
\end{array} \\
& 0.0321520000000000 \quad 0.0724240000000000] \text { '; \%Ohms }
\end{aligned}
$$

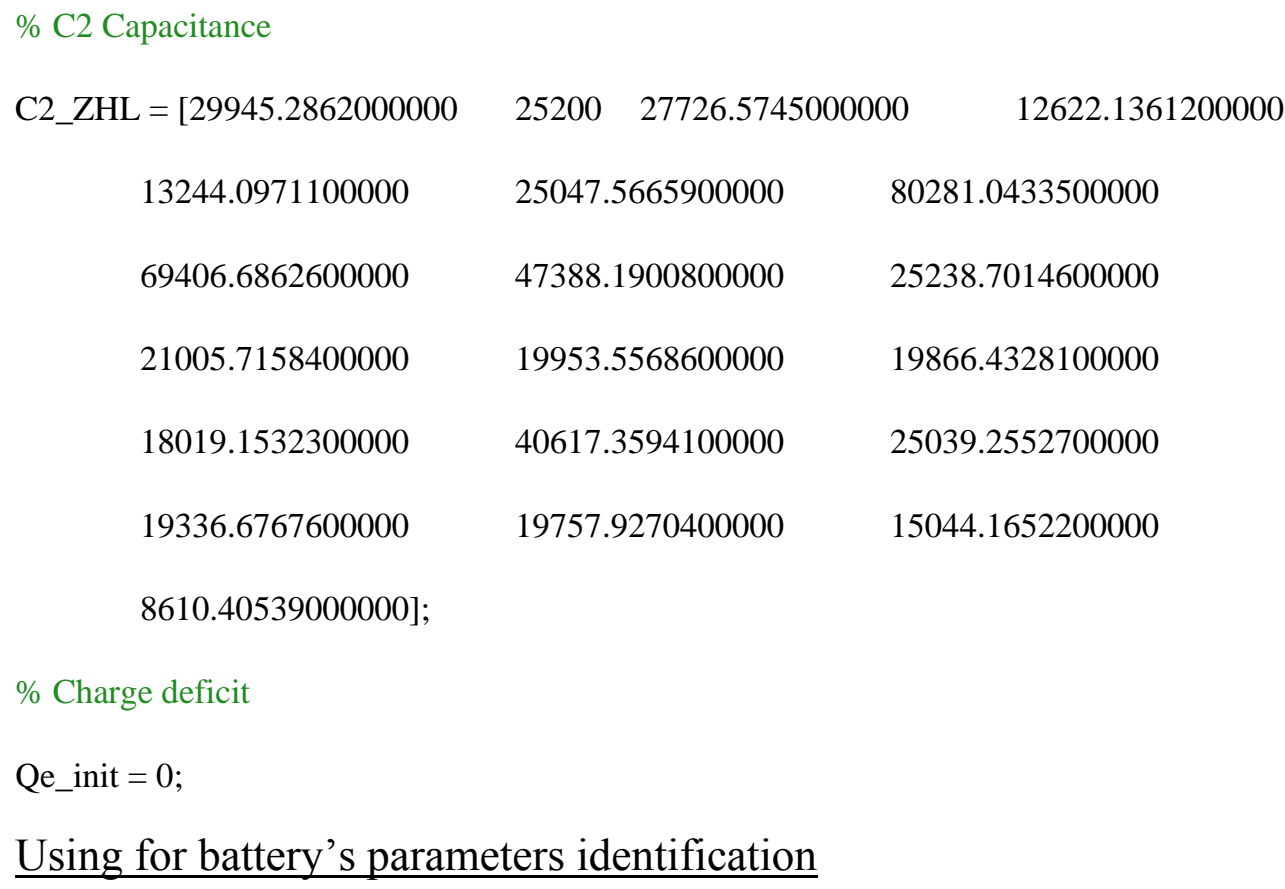


end

Uprevious=voltage_cell1(i);

Icurrent=Current_cell1(i+1);

Iprevious=Current_cell1(i);

Unow (i) $=\mathrm{W} 1(\mathrm{i}) *$ Uprevious $+\mathrm{W} 2(\mathrm{i}) *$ Icurrent+W2(i)*Iprevious+W2(i);

error=Unow(i)-voltage_cell1(i);

W1(i+1)=W1(i)-n*Unow(i)*error+bita*(W1(i)-W1(i-1));

W2(i+1)=W2(i)-n*Icurrent*error+bita*(W2(i)-W2(i-1));

$\mathrm{W} 2(\mathrm{i}+1)=\mathrm{W} 2(\mathrm{i})-\mathrm{n} *$ Iprevious*error+bita*(W2(i)-W2(i-1));

W2(i+1)=W2(i)-n*error+bita*(W2(i)-W2(i-1);

end 